\title{
The Electrical Aftermath: Brain Signals of Posttraumatic Stress Disorder Filtered Through a Clinical Lens
}

\author{
Mamona Butt ${ }^{1 \dagger}$, Elizabeth Espinal ${ }^{1 \dagger}$, Robin L. Aupperle ${ }^{2,3}$, Valentina Nikulina ${ }^{1,4}$ \\ and Jennifer L. Stewart ${ }^{2,3 *}$
}

${ }^{1}$ Department of Psychology, Queens College, City University of New York, Flushing, NY, United States, ${ }^{2}$ Laureate Institute for Brain Research, Tulsa, OK, United States, ${ }^{3}$ Department of Community Medicine, Oxley College of Health Sciences, University of Tulsa, Tulsa, OK, United States, ${ }^{4}$ Department of Psychology, The Graduate Center, City University of New York, New York, NY, United States

OPEN ACCESS

Edited by:

Agorastos Agorastos, Aristotle University of Thessaloniki,

Greece

Reviewed by:

Payam Norouzzadeh, North Carolina State University, United States

Tara Thiagarajan, Sapien Labs,

United States

${ }^{*}$ Correspondence:

Jennifer Lorraine Stewart jstewart@/aureateinstitute.org

tThese authors have contributed equally to this work.

Specialty section: This article was submitted to Mood and Anxiety Disorders, a section of the journal Frontiers in Psychiatry

Received: 29 January 2019 Accepted: 13 May 2019 Published: 31 May 2019

Citation: Butt M, Espinal E, Aupperle RL, Nikulina $V$ and Stewart JL (2019) The Electrical Aftermath:

Brain Signals of Posttraumatic Stress Disorder Filtered Through a Clinical Lens.

Front. Psychiatry 10:368. doi: 10.3389/fpsyt.2019.00368
This review aims to identify patterns of electrical signals identified using electroencephalography (EEG) linked to posttraumatic stress disorder (PTSD) diagnosis and symptom dimensions. We filter EEG findings through a clinical lens, evaluating nuances in findings according to study criteria and participant characteristics. Within the EEG frequency domain, greater right than left parietal asymmetry in alpha band power is the most promising marker of PTSD symptoms and is linked to exaggerated physiological arousal that may impair filtering of environmental distractors. The most consistent findings within the EEG time domain focused on event related potentials (ERPS) include: 1) exaggerated frontocentral responses (contingent negative variation, mismatch negativity, and P3a amplitudes) to task-irrelevant distractors, and 2) attenuated parietal responses (P3b amplitudes) to task-relevant target stimuli. These findings suggest that some individuals with PTSD suffer from attention dysregulation, which could contribute to problems concentrating on daily tasks and goals in lieu of threatening distractors. Future research investigating the utility of alpha asymmetry and frontoparietal ERPs as diagnostic and predictive biomarkers or intervention targets are recommended.

Keywords: posttraumatic stress disorder, trauma, electroencephalography, event related potentials, brain asymmetry

\section{POSTTRAUMATIC STRESS AND THE BRAIN: THE ELECTRICAL AFTERMATH}

Approximately 6 out of 100 people in the U.S. will suffer from posttraumatic stress disorder (PTSD) during their lifetime, an illness linked to significant distress, disability, and social/emotional impairment (1). Although theoretical and data-driven approaches suggest that PTSD is linked to alterations in brain circuitry that involve subcortical reactivity to trauma-related memories, thoughts, and emotions in addition to impaired prefrontal emotion regulation and inhibitory control (2-5), there are still gaps in our knowledge that neuroimaging tools can be used to address. This includes: 1) What is the extent of perceptual, cognitive, and emotional alterations as a function of trauma symptoms with respect to processing delays and allocation of brain resources? 2) To 
what extent do these brain alterations predict future course of disorder as well as treatment success or failure? and 3) Can these particular alterations be targeted by psychological and/or biological treatment approaches? A goal of clinical neuroscience is to develop precision medicine for individualized monitoring and treatment of clinical symptoms (6). With respect to treatment dissemination, it would be ideal if symptom and treatment evaluation tools were inexpensive and widely available. In this respect, electroencephalography (EEG) is an ideal methodology for moving the field towards a neuroscience-based, precision medicine approach to the treatment of PTSD.

Clinical neuroscientists often employ EEG, a relatively inexpensive yet powerful neuroimaging tool, to measure electrical brain signals in hopes of uncovering mechanisms and circuitry that are disrupted as a function of PTSD that can in turn be utilized in prevention, screening, and intervention efforts. Employing EEG methodology in clinical populations is beneficial for several reasons. First, EEG is a non-invasive, easy to administer technique that measures electrical signals on the scalp arising from pyramidal neurons firing within the cortex. Second, EEG possesses excellent temporal resolution on the order of milliseconds, facilitating study of early perceptual, attentional, and cognitive/emotional processes that may be derailed as a function of disorder. Third, comprehensive large-scale EEG electrode configurations are now available, providing improved spatial resolution to localize these electrical signals within the brain, elucidating where and when mental processes occur and how they are disrupted in particular clinical populations. Fourth, as mobile EEG systems are also available, recording of electrical signals can be routinely employed in clinical inpatient/outpatient, hospital, and community settings to assess changes as a function of symptom severity or improvement.

The purpose of this review is to evaluate what EEG research has taught us thus far with respect to brain circuitry and processes disrupted as a function of traumatic events and PTSD, results that we term "the electrical aftermath" of trauma, and then suggest future directions to further explore the unanswered questions presented above. To this end, we first define trauma and PTSD, explain how these constructs are typically measured within the EEG literature, and present clinical issues relevant to EEG studies of PTSD. Second, we evaluate studies of trauma and PTSD in both frequency and time domains of EEG recording, highlighting consistent findings while casting a floodlight on clinical and methodological conundrums warranting further consideration in future studies. Lastly, we discuss research evaluating EEG signals as potential treatmentrelevant biomarkers, and the research that is necessary for moving towards the use of EEG to enhance treatment outcomes for PTSD.

\section{DEFINING TRAUMA AND PTSD}

As the majority of the studies discussed within this review define PTSD on the basis of Diagnostic and Statistical Manual of Mental Disorders-IV (DSM-IV) criteria, we will first outline these criteria and then elaborate upon recent DSM-5 revisions.
PTSD is the primary disorder characterized by exposure to a traumatic event, wherein one encounters or witnesses actual or threatened death or severe injury and reacts with intense fear, helplessness, and/or horror (7). Traumatic events include child abuse (physical, sexual, emotional), child neglect, domestic violence, physical/sexual assault, accidents, life-threatening illness, death of a family member, natural disasters, war, combat, and community or school violence (8). Other DSM-IV PTSD criteria include at least one symptom of recurrent and intrusive thoughts (e.g., flashbacks), three or more symptoms of persistent avoidance and numbing of stimuli related to the trauma (e.g., avoiding thoughts and activities), and two or more symptoms of increased arousal (e.g., hypervigilance and difficulty falling/staying asleep) for at least 1 month. Symptoms of re-experiencing include recurrent nightmares, physical responses to trauma cues, and/or sensations that the trauma is continuing. For example, Iraqi veterans may have frequent nightmares of their experiences in Iraq, sweating every time they remember particular situations such as bombings; additionally, veterans may avoid visiting places similar to Iraq to avoid aversive feelings, memories, and physiological sensations linked to these violent attacks. A PTSD diagnosis requires that symptoms cause significant disturbance and impairment to social, vocational, and other imperative life functioning (7). PTSD criteria in DSM-5 (9) were updated by transforming these three clusters (re-experiencing, avoidance/numbing, and arousal) into four symptom clusters present for 1 month or longer: 1) intrusions; 2) avoidance of trauma-related thoughts or activities; 3) negative alterations in cognition and mood (e.g., negative affect, anhedonia, self- and other-blame, inability to recall details of the trauma, and isolation); and 4) changes in arousal (e.g., hypervigilance, exaggerated startle response, irritability, aggression, sleep problems, and concentration difficulties). One important issue to consider with respect to categorical classification of disorder is that there are many paths to a PTSD diagnosis, and symptom heterogeneity is the rule, not the exception $(10,11)$. As a result, in addition to evaluating brain activity as a function of presence versus absence of a PTSD diagnosis, relating brain processes to dimensional symptom presentations in line with the Research Domain Criteria may facilitate more rapid development of precision medicine for treatment of trauma-related dysfunction (12-14).

\section{CLINICALLY RELEVANT ISSUES TO EXPLORE}

A primary aim of clinical neuroscience research is to identify brain circuitry and/or processes (often termed "biological markers" or "biomarkers") that differentiate individuals with and without a particular illness or condition of interest. Three biomarker types are especially relevant to trauma neuroscience: 1) diagnostic, identifying people with a specific disorder diagnosis (or subtype of disorder); 2) predictive, identifying people who will improve or worsen as a result of treatment; and 3) prognostic, identifying people who relapse or experience changes in clinical severity (15). Traditionally, researchers advocated for diagnostic metrics of 
disorder that were specific (i.e., differentiating those with PTSD from healthy individuals without these symptoms) and sensitive (i.e., discerning those with PTSD from individuals diagnosed with other disorders); biomarkers could also be conceptualized as state-like (evident only in people meeting criteria for current PTSD) or trait-like (present in people who have ever suffered from PTSD, regardless of current symptom status). However, in line with the dimensional Research Domain Criteria (RDoC) framework, biomarkers may index clinical symptoms that transcend diagnostic boundaries (e.g., concentration difficulties, negative affect, and sleep disturbances) and that may predict illness course (prognostic biomarkers) and treatment outcome (predictive biomarkers). Biomarker strength may also vary as a function of biological differences such as sex or age. Comparing patterns of brain activity between individuals diagnosed with PTSD and healthy individuals who have experienced similar traumatic events may help to identify what brain mechanisms capture clinically heightened responses to trauma (not just the experience of trauma itself). Moreover, it is useful to identify whether patterns of electrical signals are a result of a particular type of trauma (e.g., combat) or are present across various types of trauma (e.g., sexual assault, childhood neglect, car accidents, natural disasters).

It is unlikely that a diagnostic biomarker of "pure PTSD" exists, given that PTSD symptoms are also present in major depressive disorder (MDD) (negative, affect, anhedonia, sleep/concentration problems), panic disorder (PD) (negative affect, hyperarousal), and generalized anxiety disorder (GAD) (negative affect, irritability, sleep/concentration difficulties); in addition, avoidance and negative affect associated with reactions to trauma in PTSD are often accompanied by coping strategies involving substance use, thereby complicating the clinical picture $(16,17)$. Individuals suffering from PTSD have approximately twice the odds of meeting criteria for comorbid $\mathrm{PD}, \mathrm{GAD}$, and MDD than those without the disorder (1). Prior work indicates that although PTSD and MDD share symptoms of distress, they are distinct disorders, and concurrent experience of both is associated with greater re-experiencing and negative affect than PTSD alone (18). Given symptom overlap and high comorbidity, it is essential for clinical neuroscience work to explicitly address potentially comorbid symptoms and disorders to address biomarkers of transdiagnostic symptoms (e.g., anhedonia, anxiety) as opposed to just PTSD-specific symptoms (e.g., intrusions involving nightmares and flashbacks). The majority of EEG studies discussed below investigate potential diagnostic biomarkers of PTSD and trauma symptoms; less electrophysiology work has focused on proposed prognostic or predictive biomarkers within the context of trauma treatment or symptom course over time.

Similarly, with regard to state versus trait prognostic and diagnostic biomarkers, it is helpful for neuroscience research to determine whether current/past symptom status, current medication/treatment status, and time since the traumatic event moderates relationships between brain mechanisms and PTSD. Finally, concerning potential sex differences, PTSD is more prevalent in women than men (1) and it is possible that differences in stress-related biological processes may clarify sex imbalances in PTSD prevalence (19). Given these concerns, the present review identifies the degree to which EEG studies of PTSD and trauma more broadly address issues of comorbidity, medication status, time elapsed since the traumatic event, and sex differences within clinical groups as well as degree of trauma experienced in healthy comparison subjects. Evaluation of these factors enable us to provide recommendations for future PTSD biomarker refinement and testing. Before relating clinical symptoms to electrophysiology, however, we first explain how trauma and PTSD are quantified within this literature.

\section{CLINICAL CAPTURE OF TRAUMA AND POSTTRAUMATIC STRESS DISORDER}

Neuroimaging studies utilize self-report scales and/or interviews to quantify duration and severity of trauma-related symptoms and relate them to brain function. Table 1 outlines measures often employed in EEG studies to index trauma and PTSD symptoms. With respect to categorizing presence versus absence of PTSD diagnosis, researchers typically employ one or both of the following: 1) the Clinician Administered PTSD Scale (CAPS) $(24,25)$, considered the "gold standard" assessment of PTSD symptom severity (26); and 2) the PTSD module of Structured Clinical Interview for DSM-IV (SCID) (27). Alternatively, some studies utilize the PTSD Checklist (PCL), a brief self-report questionnaire that is highly correlated with the CAPS $(r>.90)$ in place of a clinical interview; three versions assess trauma symptoms in specific (PCL-S), civilian (PCL-C), and military (PCL-M) samples $(28,29)$.

Additional measures of PTSD symptoms utilized by EEG researchers include: 1) the Davidson Trauma Scale (DTS), a brief questionnaire that possesses high concurrent validity with DSM-IV PTSD symptoms elicited by the SCID (30); 2) the Impact of Event Scale-Revised (IES-R) (31), a self-report measure highly correlated with the PCL $(r>.80)$ assessing frequency of intrusion, avoidance, and hyperarousal associated with trauma $(32,33)$; and 3 ) the PTSD Symptom Scale (PSS), a self-rated scale indexing PTSD symptoms over the past 2 weeks (34). On the whole, diagnosing PTSD via the CAPS and is advantageous over the SCID, which is typically administered in its entirety (including multiple modules for various psychotic, mood, anxiety, and substance use disorders (SUDs), not just the PTSD module) as well as the PSS, which captures only a 2 -week timeframe (when a month of symptoms are required to meet criteria for PTSD).

To address trauma encountered during childhood and adolescence, three questionnaires have been utilized in the EEG literature: 1) the Early Life Stress Questionnaire (ELSQ), which queries experience of adverse childhood events including abuse, neglect, natural disasters, major illness, adoption, poverty, and domestic violence (35); 2) the Childhood Trauma Questionnaire (CTQ), which focuses on multiple facets of abuse and neglect (36); and 3) the Childhood Experiences of Victimization Questionnaire (CEVQ), which evaluates peer bullying, corporal punishment, 
TABLE 1 | Measures of posttraumatic stress disorder (PTSD), trauma, and resilience employed in electroencephalography (EEG) research.

\begin{tabular}{|c|c|}
\hline Measure & Description \\
\hline $\begin{array}{l}\text { Clinician-Administered PTSD Scale } \\
\text { (CAPS })^{1}\end{array}$ & $\begin{array}{l}30 \text { items. Measures onset/duration of past week, current month, and lifetime PTSD symptoms, subjective distress, and social } \\
\text { functioning. Scores include three subscales: (1) re-experiencing (five items); (2) avoidance/numbing (nine items); and } \\
\text { (3) hyperarousal (six items). CAPS-5 has been adapted for DSM-5 (20). }\end{array}$ \\
\hline Structured Clinical Interview for & 20 items. Queries current and past: (1) exposure and reactions to traumatic event; (2) three clusters of symptoms: \\
\hline DSM-IV (SCID), PTSD module1 & re-experiencing, avoidance, hyperarousal; (3) symptom duration; and (4) impairment. SCID-5 has been adapted for DSM-5 (21). \\
\hline Davidson Trauma Scale (DTS)² & $\begin{array}{l}17 \text { items. Measures frequency of DSM-IV PTSD symptoms using a five-point scale, } 0 \text { (not at all) to } 4 \text { (everyday). A severity score } \\
\text { can also be calculated, and total score can be computed by summing severity and frequency scores. Subscale scores can be } \\
\text { calculated for re-experiencing/intrusion, avoidance/numbness, and hyperarousal. }\end{array}$ \\
\hline $\begin{array}{l}\text { Structured Interview for PTSD } \\
\text { (SI-PTSD) }\end{array}$ & $\begin{array}{l}17 \text { items. Indexes frequency and severity of PTSD symptoms over the past } 4 \text { weeks and during the worst period ever, and is } \\
\text { unique in that it includes questions regarding suicidal ideation and guilt. }\end{array}$ \\
\hline PTSD Checklist (PCL)² & $\begin{array}{l}17 \text { items. Questionnaire assesses DSM-IV PTSD symptoms, often used to diagnose and/or track symptom changes as a } \\
\text { function of treatment. Three versions of the PCL can be administered: civilian (PCL-C), military (PCL-M), and specific (PCL-S). } \\
\text { Respondents use a five-point Likert scale when endorsing responses, from } 1 \text { (not at all) to } 5 \text { (extremely). Responses from all } \\
\text { items are combined for a total severity score. PCL- } 5 \text { has been adapted for DSM-5 (22). }\end{array}$ \\
\hline PTSD Symptom Scale (PSS) $)^{1,2}$ & $\begin{array}{l}17 \text { items. Questionnaire and interview versions assessing DSM-IV PTSD symptom severity over the past } 2 \text { weeks, as compared } \\
\text { to other questionnaires, which measure for } 1 \text { month. Queries about a specific, single trauma and items assess re-experiencing } \\
\text { (4), avoidance (7), and hyperarousal (6). Provides ratings for each item ranging from } 0 \text { to 3, from } 0 \text { (not at all) to } 3 \text { (five or more } \\
\text { times). The PSS-I-5 (interview version) has been adapted to DSM-5 (23). }\end{array}$ \\
\hline $\begin{array}{l}\text { Childhood Trauma Questionnaire } \\
(\text { CTQ })^{2}\end{array}$ & $\begin{array}{l}28 \text { items. Measures the following traumatic maltreatment experiences: (1) emotional abuse; (2) sexual abuse; (3) physical abuse; } \\
\text { (4) emotional neglect; and (5) physical neglect. Participants answer on a Likert scale ranging from } 1 \text { (never) to } 5 \text { (very often); } \\
\text { subscores are then calculated for each type of maltreatment. }\end{array}$ \\
\hline $\begin{array}{l}\text { Childhood Experiences of } \\
\text { Victimization Questionnaire } \\
(\text { CEVQ)2 }\end{array}$ & $\begin{array}{l}18 \text { items. Measures various types of victimization including bullying (peer to peer), witnessing domestic violence, emotional, } \\
\text { physical, and sexual abuse, as well as corporal punishment. }\end{array}$ \\
\hline Combat Exposure Scale (CES)² & $\begin{array}{l}7 \text { items. Assesses degree of stress combatants endured during wartime on a five-point frequency Likert scale }(0=\text { no or never } \\
\text { and } 5=\text { more than } 50 \text { times), four-point frequency }(0=\text { no to } 4=\text { more than } 12 \text { times), five-point duration }(1=\text { never to } 5=\text { more } \\
\text { than } 6 \text { months), or four-point degree of loss ( } 1=\text { no one to } 4=\text { more than } 50 \%) \text { scales. }\end{array}$ \\
\hline $\begin{array}{l}\text { Impact of Event Scale - Revised } \\
(\text { IES-R })^{2}\end{array}$ & $\begin{array}{l}22 \text { items. Measures the frequency of intrusions (eight items), avoidance (eight items), and hyperarousal (six items) associated with } \\
\text { experienced trauma. }\end{array}$ \\
\hline $\begin{array}{l}\text { Mississippi Scale for Combat- } \\
\text { Related PTSD (M-PTSD)2 }\end{array}$ & $\begin{array}{l}35 \text { items. Indexes symptom frequency of combat-related DSM-IV PTSD and other associated disorders (depression/suicidality/ } \\
\text { substance use) in veterans and active service military. Individuals how they feel for each item on a five-point Likert scale. }\end{array}$ \\
\hline $\begin{array}{l}\text { Early Life Stress Questionnaire } \\
(E L S Q)^{2}\end{array}$ & $\begin{array}{l}19 \text { items. Queries experience of various adverse childhood events, including sexual abuse, natural disasters, major illness, } \\
\text { domestic violence, poverty, and neglect. }\end{array}$ \\
\hline
\end{tabular}

${ }^{1}$ Clinician interview. 2Self-report questionnaire. DSM-IV, Diagnostic and Statistical Manual of Mental Disorders, fourth edition; DSM-5, Diagnostic and Statistical Manual of Mental Disorders, fifth edition.

domestic violence, and facets of abuse (emotional, physical, and sexual) (37). In contrast to early life trauma, two scales capture traumatic symptoms suffered as a result of military service: 1) the Mississippi Scale for Combat-Related PTSD (M-PTSD) queries DSM-IV PTSD symptoms as well as comorbid substance use, suicide, and depression (38); and 2) the Combat Exposure Scale (CES) is a short questionnaire determining degree of trauma experienced in the military (from "light" to "heavy") that is moderately correlated with M-PTSD (39).

\section{NAVIGATING ELECTROENCEPHALOGRAPHY RESULTS WITHIN A CLINICAL FRAMEWORK}

Table 2 provides a summary of effect size magnitudes of studies reporting a statistically significant (most often $p<$ .05) relationship between trauma/PTSD symptoms, measured categorically and/or dimensionally, and various EEG metrics. In addition to this global summary, Tables 3-5 highlight particular facets of EEG study design relevant to clinical capture of trauma and PTSD. The column labeled Trauma Group lists the sample size for the group of interest, whereas Trauma Type characterizes trauma experienced within this group. Additionally the PTSD DX column lists measures used by each EEG study to assess trauma and/or PTSD diagnosis/ symptoms, whereas Control Group Trauma Exposure lists the sample size for control subjects with and without trauma exposure. The Clinical Controls column explains whether each study excluded trauma participants with medical problems $(\mathrm{M})$, neuropsychological problems $(\mathrm{N})$, and psychiatric $(\mathrm{P})$ problems other than PTSD. Moreover, we highlight which studies excluded participants with suicidal ideation (SUIC) as well as specific comorbid disorders such as anxiety disorders (ANX), attention-deficit hyperactivity disorder (ADHD), depression (DEP), psychosis (PSY), and SUDs. In this column, we also show which studies explicitly addressed: 1) medication effects (MED) in their trauma group; 2) time since the traumatic event occurred (TIME); and 3) sex differences within their sample (SEX). Next, the Symptom Correlations column highlights whether researchers correlated dimensional symptom scales with patterns of brain activity, and if so, what scale was used. Finally, the Results column 
TABLE 2 | Effect sizes for significant electroencephalography (EEG) and event related potential (ERP) results demonstrating categorical and dimensional capture of trauma and posttraumatic stress disorder (PTSD).

\section{Categorical:} trauma/PTSD vs control

\section{EEG frequency}

Frontal asymmetry

Parietal asymmetry

Theta power

Alpha power/peak

frequency

Beta connectivity

Gamma connectivity

Auditory attention and working memory

P2 amplitude

P2 latency

N2 amplitude

N2 latency

P3 amplitude

3 latency

Mismatch negativity

(MMN) amplitude

Auditory inhibition

P3 Latency

Visual attention and working memory
P2 Amplitude - Medium (64)

3 amplitude

Late positive

potential (LPP)

amplitude
- Small (40);

- Medium-large (41)

- Medium-large (42)

- Large (43)

- Small (40)

- Medium (43)

- Large (42)

- Medium (46)

- Small (46)

- Medium (47)

- Large (48)

- Large (48)

- Medium (49)

- Large (50)

- Medium-large (49)

- Medium (49)

- Large (50)

- Small (52)

- Large (50)

- Medium (50)

- Medium (49)

- Medium-large (53)

- Large (54)

- Large (55)

- Large (56)

- Large (57)

- Large (50)

- Large (61)

- Medium-large (63)

- Medium (65)

- Large (66)

- Large (67)

- Small (68)

- Small-medium (69)

- Medium (70)

- Medium (71)

- Large (72)

- Large (73)

- Large (74)

- Large (72)

- Large (68)

- Medium-large (68)

- Medium (66)

- Medium (77)

\begin{tabular}{lll}
$\begin{array}{l}\text { Contingent negative } \\
\text { variation (CNV) }\end{array}$ & $\bullet$ Large (82) & $\bullet$ Small-medium (82) \\
$\begin{array}{l}\text { amplitude } \\
\text { N170 amplitude }\end{array}$ & $\bullet$ Small (83) & \\
& $\bullet$ Medium (84) & \\
\hline
\end{tabular}

Dimensional:

correlates with trauma and/or PTSD symptoms

- Small-medium (42)

- Medium (42)

- Medium-large (43)

- Large (44)

- Medium (42)

- Large (45)

N/A

- Small-medium (47)

- Medium (48)

- Medium (48)

- Medium (49)

- Large (51)

N/A

N/A

N/A

- Medium (58)

- Medium (59)

- Large (54)

- Large (50)

- Large (60)

N/A

- Large (62)

N/A

N/A

- Large (75)

- Large (76)

- Medium (76)

- Small $(78,79)$

- Small (80)

- Medium $(78,79)$

- Medium (81)

- Large (66)

- Large (75)

(Continued)
TABLE 2 | Continued

\begin{tabular}{|c|c|c|}
\hline & $\begin{array}{l}\text { Categorical: } \\
\text { trauma/PTSD vs control }\end{array}$ & $\begin{array}{l}\text { Dimensional: } \\
\text { correlates with trauma } \\
\text { and/or PTSD symptoms }\end{array}$ \\
\hline N4 amplitude & $\mathrm{N} / \mathrm{A}$ & - Medium (86) \\
\hline P6 amplitude & - Medium (77) & $\mathrm{N} / \mathrm{A}$ \\
\hline \multicolumn{3}{|l|}{ Visual inhibition } \\
\hline P2 amplitude & $\begin{array}{l}\text { - } \text { Medium (87) } \\
\text { - Large (88) }\end{array}$ & $\mathrm{N} / \mathrm{A}$ \\
\hline N2 Latency & - Large (89) & $\mathrm{N} / \mathrm{A}$ \\
\hline P3 amplitude & $\begin{array}{l}\text { - Large (90) } \\
\text { - Large (57) } \\
\text { - Large (88) }\end{array}$ & - Large (90) \\
\hline P3 latency & $\begin{array}{l}\text { - Large (57) } \\
\text { - Large (91) }\end{array}$ & $\begin{array}{l}\text { - Medium (92) } \\
\text { - Medium-large (91) }\end{array}$ \\
\hline $\begin{array}{l}\text { Error-related } \\
\text { negativity (ERN) } \\
\text { amplitude }\end{array}$ & $\mathrm{N} / \mathrm{A}$ & $\begin{array}{l}\text { - Small (93) } \\
\text { - Small (94) }\end{array}$ \\
\hline LPP amplitude & - Small (95) & $\mathrm{N} / \mathrm{A}$ \\
\hline \multicolumn{3}{|c|}{ Emotion regulation and reward processing } \\
\hline LPP amplitude & - Medium (96) & $\mathrm{N} / \mathrm{A}$ \\
\hline
\end{tabular}

The following benchmarks were used to categorize effect size magnitudes: 1) Cohen's $d$ and Hedge's g: small $=0.2$, medium $=0.5$, and large $=0.8$; 2) Partial $\eta^{2}$ and $R^{2}$ : small $=.01$, medium $=.09$, and large $\left.=.25 ; 3\right) \eta^{2}$ : small $=.01$, medium $=.06$, and large $=.14$; and 4) Spearman's $\rho$ : small $=0.1$, medium $=0.3$, and large $=0.5$. Studies with null findings or no effect size data are not included in this table, but are included in Tables 3-5. N/A, Not applicable.

explains findings for each study, listing significance values, and effect sizes, if they were provided or if studies included means and standard deviations, thereby enabling us to calculate effect sizes. Effect sizes reported include Cohen's $d$, Hedges $g$, partial $\eta^{2}, \eta^{2}, R^{2}$, and Spearman's $\rho$. Asterisks next to the author name and reference $\left({ }^{*}\right)$ highlight studies that did not provide enough information for us to calculate effect sizes for at least one significant result.

\section{ELECTROENCEPHALOGRAPHY MEASURED IN FREQUENCY AND TIME: WHAT IS THE DIFFERENCE?}

EEG signals represent relative electrical potentials acquired over time at sensors placed on the scalp while an individual is in an uncontrolled resting state or an active task session. EEG signals can then be extracted for data analysis in the frequency domain, illustrated in Figure 1, and/or the time domain, illustrated in Figure 2. The spectral composition, or power spectrum, of the signal is then estimated using various fast Fourier transform algorithms. The most common metric employed in analysis is power within a particular frequency band. Differences in power between hemispheres are also quantified by calculating an asymmetry score metric. Additionally, peak frequency values within a band (identifying the frequency with the highest amplitude per individual) and connectivity (amplitude or power correlations between electrodes located in various scalp locations) can be quantified. EEG data are typically analyzed in the time domain by time-locking electrical signals elicited to a particular stimulus or response and then averaging these 
TABLE 3 | Electroencephalographic (EEG) frequency band studies of trauma and/or posttraumatic stress disorder (PTSD).

\begin{tabular}{|c|c|c|c|c|c|c|c|c|}
\hline $\begin{array}{l}\text { Author } \\
\text { (Ref.) }\end{array}$ & $\begin{array}{l}\text { Trauma } \\
\text { group } \\
\text { (T) }\end{array}$ & $\begin{array}{l}\text { Trauma } \\
\text { type }\end{array}$ & $\begin{array}{l}\text { PTSD } \\
\text { DX }\end{array}$ & $\begin{array}{l}\text { Control } \\
\text { group (C) } \\
\text { trauma } \\
\text { exposure? }\end{array}$ & $\begin{array}{l}\text { Clinical } \\
\text { controls }\end{array}$ & $\begin{array}{l}\text { Symptom } \\
\text { correlations }\end{array}$ & Ref/Design & Results \\
\hline $\begin{array}{l}\text { Bangel et } \\
\text { al. }(61)^{*}\end{array}$ & 13 & $\begin{array}{l}\text { PTSD } \\
(\text { UNKN) }\end{array}$ & CAPS & $13 Y C$ & $\begin{array}{l}\text { DEP; N; P; } \\
\text { SUIC }\end{array}$ & Y (CAPS) & $\begin{array}{l}\text { AVG; two-stimulus } \\
\text { auditory oddball } \\
\text { (30 min) }\end{array}$ & $\begin{array}{l}T>Y C \text { frontal theta power }(p<.05) \\
T>Y C \text { right parietal alpha power } \\
\text { suppression }(p<.05)\end{array}$ \\
\hline $\begin{array}{l}\text { Begić et al. } \\
(97)^{\star}\end{array}$ & 20 & $\begin{array}{l}\text { PTSD } \\
\text { (COMBAT) }\end{array}$ & CAPS & $20 \mathrm{NC}$ & MED; N; P & $N$ & $\begin{array}{l}\text { LM; resting }(10 \mathrm{~m} \text {, } \\
\mathrm{EC})\end{array}$ & $\begin{array}{l}\mathrm{T}>\mathrm{NC} \text { central theta }(p<.01) ; \mathrm{T}>\mathrm{NC} \\
\text { frontal, central, and occipital beta } \\
(p<.01) ; \mathrm{T}=\mathrm{C} \text { alpha and delta }(p>.05)\end{array}$ \\
\hline $\begin{array}{l}\text { Cowdin et } \\
\text { al. }(98)^{\star}\end{array}$ & 17 & PTSD (VAR) & $\begin{array}{l}\text { CAPS; } \\
\text { SCID }\end{array}$ & 13 YC & MED; N; P & $N$ & $\begin{array}{l}\text { UNKN; REM (650s, } \\
\text { EC) }\end{array}$ & $\begin{array}{l}Y C>T \text { right frontal early theta power } \\
(p=.04) \text { and bilateral frontal late } \\
\text { theta power (no } p \text { value reported) }\end{array}$ \\
\hline $\begin{array}{l}\text { Curtis and } \\
\text { Cicchetti } \\
\text { (40) }\end{array}$ & 44 & $\begin{array}{l}\text { Trauma } \\
\text { (CHILD) }\end{array}$ & $\mathrm{N} / \mathrm{A}$ & $43 \mathrm{NC}$ & MED; SEX & $N$ & $\begin{array}{l}\text { LM; Resting ( } 8 \mathrm{~m} \text {, } \\
\mathrm{EC} \text { and } \mathrm{EO})\end{array}$ & $\begin{array}{l}\mathrm{T}>\mathrm{NC} \text { right parietal asymmetry } \\
\left(p=.06 . \text { partial } \eta^{2}=.04\right) \text {; men: } \mathrm{T}> \\
\mathrm{NC} \text { left frontal }\left(p<.05 \text {, partial } \eta^{2}=.07\right) \\
\text { and occipital }\left(p<.05 \text {, partial } \eta^{2}=.09\right) \\
\text { asymmetry; women: resilient } \mathrm{NC}>\mathrm{T} \\
\text { right frontal asymmetry }(p<.05 \text {, partial } \\
\left.\eta^{2}=.06\right)\end{array}$ \\
\hline $\begin{array}{l}\text { Ehlers et al. } \\
(99)^{\star}\end{array}$ & 19 & PTSD (VAR) & SSAGA & 39 YC & $\mathrm{N} / \mathrm{A}$ & $N$ & $\begin{array}{l}\text { Bipolar; resting } \\
\text { (10-15m EC) }\end{array}$ & $\begin{array}{l}\text { T > YC gamma power }(p<.004) ; T> \\
\text { YC beta power }(p=.06)\end{array}$ \\
\hline $\begin{array}{l}\text { Falconer et } \\
\text { al. (53) }\end{array}$ & 44 & $\begin{array}{l}\text { PTSD } \\
(\text { UNKN) }\end{array}$ & CAPS & $44 \mathrm{NC}$ & $\begin{array}{l}\text { N; PSY; } \\
\text { SUD }\end{array}$ & $N$ & $\begin{array}{l}\mathrm{LM} \text {; resting ( } 4 \mathrm{~m} \text {, } \\
\mathrm{EC} \text { and } \mathrm{EO})\end{array}$ & $\begin{array}{l}T=N C \text { delta, theta, alpha, beta } \\
\text { power }(p>.05)\end{array}$ \\
\hline $\begin{array}{l}\text { Hostinar et } \\
\text { al. (100) }\end{array}$ & 314 & $\begin{array}{l}\text { Trauma } \\
\text { (CHILD) }\end{array}$ & $\mathrm{N} / \mathrm{A}$ & N/A & SEX & $\begin{array}{l}\text { Y (CTQ; CES- } \\
\text { D; STAI) }\end{array}$ & $\begin{array}{l}\text { Cz; resting }(6 \mathrm{~m}, \mathrm{EC} \\
\text { and } \mathrm{EO})\end{array}$ & $\begin{array}{l}\text { No relationship between frontal } \\
\text { asymmetry and CTQ }(p=.87)\end{array}$ \\
\hline $\begin{array}{l}\text { Imperatori } \\
\text { et al. }(101)^{\star}\end{array}$ & 17 & PTSD (VAR) & UNKN & $17 \mathrm{NC}$ & MED; N; P & $N$ & $\begin{array}{l}\mathrm{LM} \text {; resting }(5 \mathrm{~m} \text {, } \\
\mathrm{EC})\end{array}$ & $\begin{array}{l}T>N C \text { frontal }(p<.05) \text { and parietal } \\
(p<.05) \text { theta activity; } T>N C \\
\text { parietal alpha connectivity }(p<.05)\end{array}$ \\
\hline $\begin{array}{l}\text { Kemp et al. } \\
(44)\end{array}$ & 14 & $\begin{array}{l}\text { PTSD } \\
(\text { UNKN) }\end{array}$ & CAPS & $\begin{array}{l}15 \mathrm{NC} ; 15 \\
\text { MDD+ }\end{array}$ & DEP; N & $\begin{array}{l}\text { Y (CAPS, } \\
\text { DASS) }\end{array}$ & $\begin{array}{l}\text { LM; resting }(2 \mathrm{~m} \text {, } \\
\mathrm{EC})\end{array}$ & $\begin{array}{l}\mathrm{T}=\mathrm{NC} \text { frontal asymmetry }(p>.05) ; \\
\mathrm{T} \text { : higher CAPS linked to greater right } \\
\text { frontal asymmetry }\left(p=.02, R^{2}=.38\right)\end{array}$ \\
\hline $\begin{array}{l}\text { Lee et al. } \\
(48)\end{array}$ & 33 & $\begin{array}{l}\text { PTSD } \\
\text { (ACCID) }\end{array}$ & SCID & $30 \mathrm{NC}$ & $\mathrm{N} / \mathrm{A}$ & $\begin{array}{l}\text { Y (DTS; SIP; } \\
\text { HAM-D) }\end{array}$ & $\begin{array}{l}\mathrm{Cz} \text {; resting }(5 \mathrm{~m} \text {, } \\
\mathrm{EC})\end{array}$ & $\begin{array}{l}\text { NC }>\text { T frontocentral beta }(p=.0002, \\
d=1.09) \text { and gamma }(p=.003, d= \\
0.91) \text { connectivity; T: higher DTS-total } \\
\left(p=.003, R^{2}=.25\right), \text { SIP arousal }(p= \\
\left..03, R^{2}=.14\right) \text {, and HAMD ( } p=.03, \\
\left.R^{2}=.14\right) \text { linked to lower connectivity }\end{array}$ \\
\hline $\begin{array}{l}\text { McFarlane } \\
\text { et al. }(35)^{\star}\end{array}$ & 214 & $\begin{array}{l}\text { Trauma } \\
\text { (ELS) }\end{array}$ & $\mathrm{N} / \mathrm{A}$ & 193 NC & $\begin{array}{l}\text { M; N; P; } \\
\text { SEX }\end{array}$ & Y (ELSQ) & $\begin{array}{l}\text { LM; resting }(4 \mathrm{~m} \text {, } \\
\mathrm{EC} \text { and } \mathrm{EO})\end{array}$ & $\begin{array}{l}\text { NC }>\text { T EO beta }(p=.007) \text {, theta } \\
(p<.001) \text {, alpha }(p=.01) \text {, and delta } \\
(p<.001) \text { power, and EC beta, theta, } \\
\text { alpha and delta power (all } p<.001)\end{array}$ \\
\hline $\begin{array}{l}\text { Metzger et } \\
\text { al. (45) }\end{array}$ & 29 & $\begin{array}{l}\text { PTSD } \\
\text { (COMBAT) }\end{array}$ & $\begin{array}{l}\text { CAPS; } \\
\text { SCID }\end{array}$ & 13 YC & MED; PSY & $\begin{array}{l}\text { Y (CAPS; } \\
\text { SCL-90R } \\
\text { depression) }\end{array}$ & $\begin{array}{l}\text { LM; resting (6m, } \\
\mathrm{EC} \text { and } \mathrm{EO})\end{array}$ & $\begin{array}{l}\text { CAPS-arousal, depression, and their } \\
\text { interaction }\left(p=.01, R^{2}=.25\right) \text { linked } \\
\text { to greater right parietal asymmetry }\end{array}$ \\
\hline $\begin{array}{l}\text { Meyer et al. } \\
(43)\end{array}$ & 24 & PTSD (VAR) & SCID & $\begin{array}{l}15 Y C ; 15 \\
\text { NC }\end{array}$ & $\begin{array}{l}\text { N; PSY; } \\
\text { SUD; } \\
\text { TIME }\end{array}$ & $\begin{array}{l}\text { Y (PCL; PSS; } \\
\text { RIQ; BDI-II) }\end{array}$ & $\begin{array}{l}\text { LM; resting }(8 \mathrm{~m} \text {, } \\
\text { EC and } \mathrm{EO}) \text {; } \\
\text { neutral image }(2 \mathrm{~m}) \text {; } \\
\text { positive image } \\
(2 \mathrm{~m}) ; \text { negative } \\
\text { image }(2 \mathrm{~m}) ; \text { trauma } \\
\text { image }(2 \mathrm{~m})\end{array}$ & $\begin{array}{l}\text { Resting: } \mathrm{T} \text { and } \mathrm{YC}>\mathrm{NC} \text { for left frontal } \\
\text { asymmetry }\left(p<.001 \text {, partial } \eta^{2}=.30\right) \text {; } \\
\mathrm{T}>\mathrm{NC} \text { for left parietal asymmetry }(p= \\
\left..04 \text {, partial } \eta^{2}=.12\right) \text {; neutral: } \mathrm{YC}>\mathrm{T} \\
\text { left frontal asymmetry }(p<.03 \text {, partial } \\
\left.\eta^{2}=.13\right) \text {; negative: } \mathrm{YC}>\mathrm{T} \text { and } \mathrm{NC} \\
\text { left frontal asymmetry ( } p<.03 \text {, partial } \\
\left.\eta^{2}=.25\right) \text {; negative: higher right frontal } \\
\text { asymmetry linked to higher PCL, PSS, } \\
\mathrm{RIQ} \text {, and BDI-Il symptoms (all } p<.01 \text {, } \\
R^{2} \text { range: .18-.32); trauma: higher right } \\
\text { frontal asymmetry linked to greater } \\
\text { emotional intensity/physical reaction to } \\
\text { image (both } p<.001, R^{2}=.24-.25 \text { ) }\end{array}$ \\
\hline $\begin{array}{l}\text { Miskovic et } \\
\text { al. (41) }\end{array}$ & 38 & $\begin{array}{l}\text { Trauma } \\
\text { (CHILD) }\end{array}$ & UNKN & $24 \mathrm{NC}$ & MED & $N$ & $\begin{array}{l}\text { AVG; resting ( } 2 \mathrm{~m} \\
\mathrm{EC} \text { and } \mathrm{EO}) 6 \\
\text { months apart }\end{array}$ & $\begin{array}{l}\mathrm{T}>\mathrm{NC} \text { right frontal asymmetry at } \\
\text { time } 1(p=.01, T>\mathrm{NC} \text { right frontal } \\
\left.\text { asymmetry at }{ }^{2}=.26\right) \text { and time } 2(p= \\
.04, \mathrm{~T}>\mathrm{NC} \text { right frontal asymmetry } \\
\left.\text { at }^{2}=.08\right)\end{array}$ \\
\hline
\end{tabular}


TABLE 3 | Continued

\begin{tabular}{|c|c|c|c|c|c|c|c|c|}
\hline $\begin{array}{l}\text { Author } \\
\text { (Ref.) }\end{array}$ & $\begin{array}{l}\text { Trauma } \\
\text { group } \\
\text { (T) }\end{array}$ & $\begin{array}{l}\text { Trauma } \\
\text { type }\end{array}$ & $\begin{array}{l}\text { PTSD } \\
\text { DX }\end{array}$ & $\begin{array}{l}\text { Control } \\
\text { group }(\mathrm{C}) \\
\text { trauma } \\
\text { exposure? }\end{array}$ & $\begin{array}{l}\text { Clinical } \\
\text { controls }\end{array}$ & $\begin{array}{l}\text { Symptom } \\
\text { correlations }\end{array}$ & Ref/Design & Results \\
\hline $\begin{array}{l}\text { Rabe, } \\
\text { Beauducel } \\
\text { et al. (42) }\end{array}$ & 43 & $\begin{array}{l}\text { PTSD } \\
\text { (ACCID) }\end{array}$ & $\begin{array}{l}\text { CAPS; } \\
\text { SCID }\end{array}$ & $\begin{array}{l}21 Y C ; 23 \\
N C\end{array}$ & $\begin{array}{l}\text { MED; N; } \\
\text { PSY; SEX; } \\
\text { SUD; } \\
\text { TIME }\end{array}$ & Y (CAPS; BDI) & $\begin{array}{l}\text { LM; resting }(8 \mathrm{~m} \text {, } \\
\text { EC and EO); } \\
\text { neutral image }(1 \mathrm{~m}) \text {; } \\
\text { positive image } \\
(1 \mathrm{~m}) ; \text { negative } \\
\text { image }(1 \mathrm{~m}) ; \text { trauma } \\
\text { image }(1 \mathrm{~m})\end{array}$ & $\begin{array}{l}\text { Trauma-related right frontal } \\
\text { asymmetry for full } \mathrm{T}(p<.09, \\
\left.\eta^{2}=.13\right) \text { and partial } \mathrm{T}\left(p<.08, \eta^{2}=\right. \\
.15) ; \text { trauma-related right parietal } \\
\text { asymmetry for full T }\left(p<.05, \eta^{2}=.19\right) \\
\text { and partial } \mathrm{T}\left(p<.05, \eta^{2}=.24\right) ; \mathrm{YC} \text { : } \\
\text { trauma-related left frontal asymmetry } \\
\left(p<.01, \eta^{2}=.36\right) \text {; higher CAPs } \\
\text { linked to greater trauma-related right } \\
\text { frontal and parietal asymmetry (all } p< \\
.05 ; R^{2} \text { range: .08-.23) }\end{array}$ \\
\hline $\begin{array}{l}\text { Rabe, } \\
\text { Zöllner et al. } \\
(102)\end{array}$ & 45 & $\begin{array}{l}\text { PTSD } \\
\text { (ACCID) }\end{array}$ & $\begin{array}{l}\text { CAPS; } \\
\text { SCID }\end{array}$ & $37 \mathrm{YC}$ & $\begin{array}{l}\text { MED; N; } \\
\text { PSY; SUD; } \\
\text { TIME }\end{array}$ & Y (PTGl) & $\begin{array}{l}\text { LM; resting ( } 8 \mathrm{~m} \text {, } \\
\mathrm{EC} \text { and } \mathrm{EO})\end{array}$ & $\begin{array}{l}\text { Higher left frontocentral asymmetry } \\
\text { linked to greater PTGI subscales (all } \\
p<.05, R^{2} \text { range: .05-. } 17 \text { ) }\end{array}$ \\
\hline $\begin{array}{l}\text { Shankman } \\
\text { et al. (103) }\end{array}$ & 32 & $\begin{array}{l}\text { PTSD } \\
\text { (ACCID, } \\
\text { ASSAULT) }\end{array}$ & CAPS & $42 \mathrm{NC}$ & SEX; TIME & $\begin{array}{l}\text { Y (CAPS; } \\
\text { DASS) }\end{array}$ & $\begin{array}{l}\text { AVG; resting ( } 4 \mathrm{~m} \text {, } \\
\mathrm{EC} \text { and } \mathrm{EO})\end{array}$ & $\begin{array}{l}\mathrm{T}=\mathrm{NC} \text { for frontal, central, and } \\
\text { parietal asymmetry (all } p>.05 \text { ) }\end{array}$ \\
\hline $\begin{array}{l}\text { Tang et al. } \\
(104)^{\star}\end{array}$ & 43 & $\begin{array}{l}\text { Trauma } \\
\text { (CHILD) }\end{array}$ & $\begin{array}{l}\text { KSADS; } \\
\text { CTQ; } \\
\text { CEVQ }\end{array}$ & N/A & DEP; MED & Y (CTQ) & $\begin{array}{l}\text { AVG; resting }(2 \mathrm{~m} \text {, } \\
E C \text { and } E O) \text { in } \\
\text { three sessions over } \\
2 \text { years }\end{array}$ & $\begin{array}{l}\text { Right frontal asymmetry interacted } \\
\text { with trauma to predict future PTSD } \\
\text { outcome }(p=.03)\end{array}$ \\
\hline $\begin{array}{l}\text { Todder } \\
\text { et al. }(105)^{\star}\end{array}$ & 10 & $\begin{array}{l}\text { PTSD } \\
(\text { UNKN })\end{array}$ & UNKN & $10 \mathrm{NC}$ & $\begin{array}{l}\text { N; PSY; } \\
\text { SUD; } \\
\text { TIME }\end{array}$ & $N$ & $\begin{array}{l}\text { AVG and LM; } \\
\text { resting }(3 \mathrm{~m}, \mathrm{EC})\end{array}$ & $\begin{array}{l}\mathrm{T}=\mathrm{NC} \text { absolute theta power } \\
(p>.05) \text {; using LORETA source } \\
\text { localization software: (1) NC > T for } \\
\text { right temporal low theta, and } 2 \text { ) } \mathrm{NC}> \\
\mathrm{T} \text { for bilateral frontal high theta (no } \\
p \text { values reported) }\end{array}$ \\
\hline $\begin{array}{l}\text { Veltmeyer } \\
\text { et al. (46) }\end{array}$ & 34 & PTSD (VAR) & CAPS & $136 \mathrm{NC}$ & N; P; TIME & Y (CAPS) & $\begin{array}{l}\text { LM; resting }(2 \mathrm{~m} \text {, } \\
\mathrm{EO})\end{array}$ & $\begin{array}{l}\mathrm{NC}>\mathrm{T} \text { low alpha power }(p=.09, \\
d=.47) ; \mathrm{NC}>\mathrm{T} \text { theta power }(p=.08, \\
d=.53) ; \mathrm{NC}>\mathrm{T} \text { high alpha power } \\
(p=.04, d=.34) \text { and theta/alpha } \\
\text { ratio }(p=.05, d=.19)\end{array}$ \\
\hline $\begin{array}{l}\text { Wahbeh } \\
\text { and Oken } \\
\text { (47) }\end{array}$ & 57 & $\begin{array}{l}\text { PTSD } \\
\text { (COMBAT) }\end{array}$ & $\begin{array}{l}\text { CAPS; } \\
\text { SCID; } \\
\text { CES }\end{array}$ & 29 YC & $\begin{array}{l}\text { N; PSY; } \\
\text { SUD }\end{array}$ & Y (CAPS; PCL) & $\begin{array}{l}\text { AVG; resting }(5 \mathrm{~m} \text {, } \\
\mathrm{EC})\end{array}$ & $\begin{array}{l}T=Y C \text { for frontal, central, parietal } \\
\text { asymmetry (all } p>.10) ; T>Y C \text { for } \\
\text { peak alpha frequency }(p<.01, d= \\
.57 \text { ); } T \text { : higher global peak frequency } \\
\text { linked to higher CAPS total/subscale } \\
\text { and } P C L \text { scores (all } p<.05 \text {, } \\
\rho \text { range: .22-.33) }\end{array}$ \\
\hline
\end{tabular}

*Effect sizes for at least one significant effect unable to be computed based on article information. ACC, traumatic accident; AVG, average reference; BD, Beck Depression Inventory; CAPS, Clinician-Administered PTSD Scale; CES, Combat Exposure Scale; CES-D, Center for Epidemiological Studies Depression Scale; CEVQ, Childhood Experiences of Victimization Questionnaire; CHILD, child abuse; CTQ, Childhood Trauma Questionnaire; DASS, Depression Anxiety Stress Scales; DEP, excluded for depression; DTS, Davidson Trauma Scale; DX, diagnosis; EC, eyes-closed recording; ELS, early life stress; ELSQ, Early Life Stress Questionnaire; EO, eyes-open recording; HAMD, Hamilton Rating Inventory for Depression; LM, linked mastoids; M, excluded for medical problems; MDD, major depressive disorder; MED, excluded for medication; N, excluded for neurological problems; N/A, not applicable; NC, trauma-no control group; ND, natural disaster; P, excluded for psychiatric diagnoses; PCL, PTSD Checklist; PGTI, Posttraumatic Growth Inventory; PSS, PTSD Symptom Scale; PSY, excluded for psychosis; Ref, reference montage; REM, rapid eye movement; RIQ, Response to Intrusions Questionnaire; SCID, Structured Clinical Interview for DSM-IV; SCL-90, Symptom Checklist 90; SEX, explicitly examined sex differences; SIP, Structured Interview for PTSD; STAI, State Trait Anxiety Inventory; SUD, excluded for substance use disorders; SUIC, excluded for suicidal ideation; T, trauma group; TIME, reported time since trauma; UNKN, unknown; VAR, various traumas; YC, trauma-yes control group.

time-locked signals over multiple trials to produce an event related potential (ERP), which amplifies signals to an event while cancelling out random noise present on individual trials. We review trauma and PTSD literature for frequency and time domains separately below. On the whole, most EEG studies within this literature record data from low-density electrode montages, limiting spatial resolution of signals beyond anterior versus posterior, or frontal, central, temporal, and parietal versus occipital scalp locations.

\section{ELECTROENCEPHALOGRAPHY FREQUENCY PATTERNS LINKED TO TRAUMA EXPERIENCE AND POSTTRAUMATIC STRESS DISORDER SYMPTOMS}

Figure 1 illustrates that continuous EEG recordings are typically examined as a function of particular frequency bands, such as 
TABLE 4 | Auditory event related potential (ERP) studies of trauma and/or posttraumatic stress disorder (PTSD).

\begin{tabular}{|c|c|c|c|c|c|c|c|}
\hline Author (Ref.) & $\begin{array}{l}\text { Trauma } \\
\text { group } \\
\text { (T) }\end{array}$ & $\begin{array}{l}\text { Trauma } \\
\text { type }\end{array}$ & PTSD DX & $\begin{array}{l}\text { Control group } \\
\text { (C) trauma } \\
\text { exposure? }\end{array}$ & Clinical controls & $\begin{array}{l}\text { Symptom } \\
\text { correlations }\end{array}$ & Results \\
\hline
\end{tabular}

\begin{tabular}{|c|c|c|c|c|c|c|}
\hline \multicolumn{7}{|c|}{ Auditory attention and working memory } \\
\hline $\begin{array}{l}\text { Araki et al. } \\
\text { (54) }\end{array}$ & 8 & $\begin{array}{l}\text { PTSD } \\
\text { (TERROR) }\end{array}$ & CAPS & $13 Y C$ & MED; N; P; TIME & $\begin{array}{l}\text { Y (CAPS; STAI } \\
\text { IES-R) }\end{array}$ \\
\hline $\begin{array}{l}\text { Bae et al. } \\
(58)^{\star}\end{array}$ & 30 & $\begin{array}{l}\text { PTSD } \\
\text { (ACCID) }\end{array}$ & SCID & $33 \mathrm{NC}$ & $\mathrm{N} ; \mathrm{P}$ & Y (SCID; DTS) \\
\hline
\end{tabular}

YC > T parietal target P3 amplitude $(p=.04, d=.81) ; \mathrm{YC}=\mathrm{T}$ for P3 latency $(p=.49)$; within T, lower P3 amplitude linked to higher CAPS avoidance $(p=$

$$
\begin{aligned}
& .04, \rho=.85) . \\
& \text { NC > T P3 amplitude }(p<.01) ; T=\text { NC }
\end{aligned}
$$

P3 latency ( $p>$.15); using P3 amplitude source analysis (LORETA): (1) higher re-experiencing linked to parietal P3 ( $p<.05, R^{2}$ range: .14-.24), (2) higher avoidance/numbing linked to higher temporal/parietal P3 and lower frontal P3 ( $p<.05, R^{2}$ range: .13-.17), and (3) higher hyperarousal linked to frontal and temporal/parietal P3 $\left(p<.05, R^{2}\right.$

\begin{tabular}{|c|c|c|c|c|c|c|c|}
\hline $\begin{array}{l}\text { Bangel et al. } \\
(61)\end{array}$ & 13 & PTSD & CAPS & $13 Y C$ & DEP; N; P; SUIC & Y (CAPS) & $\begin{array}{l}\mathrm{T}>\mathrm{YC} \text { MMN amplitude to deviants } \\
\left(p<.001, n^{2}=.63\right)\end{array}$ \\
\hline $\begin{array}{l}\text { Blomhoff et } \\
\text { al. }(106)^{\star}\end{array}$ & 11 & $\begin{array}{l}\text { PTSD } \\
\text { (FIRE) }\end{array}$ & CAPS; IES-R & $9 Y C$ & $\begin{array}{l}\text { N; PSY; SUD; } \\
\text { TIME }\end{array}$ & Y (CAPS) & $\begin{array}{l}\text { T }>\text { YC positive/negative P2-P3a } \\
\text { amplitudes linked to arousal }(p<.01) \text { and } \\
\text { avoidance }(p<.001)\end{array}$ \\
\hline $\begin{array}{l}\text { Boudarene } \\
\text { \& Timsit- } \\
\text { Berthier (55) }\end{array}$ & 19 & $\begin{array}{l}\text { PTSD } \\
(\text { UNKN) }\end{array}$ & UNKN & $17 \mathrm{YC} ; 18 \mathrm{NC}$ & TIME & $\mathrm{N}$ & $\begin{array}{l}\text { YC }>\text { T frontal P3a }(p=.0004, d=.75) \\
\text { and P3b }(p=.0001, d=.78) \text { amplitudes; } \\
\text { NC }>\text { T frontal P3a }(p=.0004, d=1.18) \\
\text { and P3b }(p=.0001, d=1.33) \text { amplitudes }\end{array}$ \\
\hline $\begin{array}{l}\text { Charles et al. } \\
(56)\end{array}$ & 16 & $\begin{array}{l}\text { PTSD } \\
\text { (ASSAULT) }\end{array}$ & SADS & $10 \mathrm{NC}$ & $\begin{array}{l}\text { M; MED; P; SEX; } \\
\text { TIME }\end{array}$ & $N$ & $\begin{array}{l}\text { NC }>\text { T central target P3 amplitude } \\
(p<.001, d=2.70) ; T=\text { NC P3 latency } \\
(p=.46)\end{array}$ \\
\hline $\begin{array}{l}\text { Falconer et } \\
\text { al. (53) }\end{array}$ & 44 & $\begin{array}{l}\text { PTSD } \\
(\text { UNKN) }\end{array}$ & CAPS & $44 \mathrm{NC}$ & N; PSY; SUD & $\mathrm{N}$ & $\begin{array}{l}\mathrm{NC}>\mathrm{T} \text { frontal }(d=.53) \text {, central }(d= \\
3.40) \text {, and parietal }(d=.70) \text { P3 target } \\
\text { amplitude }(p \leq .02)\end{array}$ \\
\hline $\begin{array}{l}\text { Felmingham } \\
\text { et al. (50) }\end{array}$ & 17 & $\begin{array}{l}\text { PTSD } \\
\text { (ASSAULT, } \\
\text { ACCID) }\end{array}$ & CAPS & $17 \mathrm{NC}$ & $\begin{array}{l}\text { DEP; MED; N; } \\
\text { PANIC; PSY; } \\
\text { SUD; TIME }\end{array}$ & Y (CAPS) & $\begin{array}{l}\text { NC }>\text { T P2 amplitude }(p<.01, d=1.17) ; \\
\text { T }>\text { NC N2 amplitude }(p<.05, d=1.24) ; \\
\text { T }>\text { NC frontal N2 latency }(p<.05, d= \\
1.20) ; \text { NC }>\text { T P3 amplitude }(p<.05, \\
d=.72) ; \text { T }>\text { NC parietal P3 latency }(p< \\
.05, d=.84) ; \text { greater numbing linked to } \\
\text { lower P3 amplitude }\left(p=.01, R^{2}=.35\right)\end{array}$ \\
\hline $\begin{array}{l}\text { Felmingham } \\
\text { et al. }(107)^{\star}\end{array}$ & 17 & $\begin{array}{l}\text { PTSD } \\
\text { (ASSAULT, } \\
\text { ACCID) }\end{array}$ & CAPS & $\begin{array}{l}12 \text { YC (ASD+); } \\
13 \text { NC (ASD-) }\end{array}$ & $\begin{array}{l}\text { DEP; MED; N; } \\
\text { PANIC; PSY; SUD }\end{array}$ & $\mathrm{N}$ & $\begin{array}{l}\text { ASD }+>\text { parietal P3b target amplitude } \\
\text { than PTSD+ and ASD }-(p<.001)\end{array}$ \\
\hline $\begin{array}{l}\text { Galletly et al. } \\
(108)^{\star}\end{array}$ & 18 & $\begin{array}{l}\text { PTSD } \\
\text { (VAR) }\end{array}$ & DIS & $18 \mathrm{NC}$ & MED; N; SUD & Y (STAl) & $\begin{array}{l}\text { T }>\text { NC N2 target latency }(p<.05) ; \mathrm{NC}> \\
\text { T P3 target amplitude }(p<.05)\end{array}$ \\
\hline $\begin{array}{l}\text { Ge et al. } \\
(109)^{\star}\end{array}$ & 13 & PTSD (ND) & PCL-C & $14 \mathrm{YC}$ & MED; N; P; SUD & $\mathrm{N}$ & T > YC MMN amplitude $(p<.05)$ \\
\hline $\begin{array}{l}\text { Hall et al. } \\
(110)\end{array}$ & 12 & $\begin{array}{l}\text { PTSD } \\
\text { (COMBAT) }\end{array}$ & $\begin{array}{l}\text { CAPS; SCID; } \\
\text { M-PTSD }\end{array}$ & $\begin{array}{l}12 \mathrm{MZ} \text { twins NC; } \\
23 \mathrm{MZ} \text { twins } \mathrm{YC} \\
23 \mathrm{MZ} \text { twins } \mathrm{NC}\end{array}$ & M & $\mathrm{N}$ & $\mathrm{T}=\mathrm{YC} / \mathrm{NC}$ P3b amplitude $(p>.05)$ \\
\hline $\begin{array}{l}\text { Kimble et al. } \\
(111)\end{array}$ & 24 & $\begin{array}{l}\text { PTSD } \\
\text { (COMBAT) }\end{array}$ & CAPS; CES & $15 Y C$ & N; SUD & $\mathrm{N}$ & $\mathrm{T}=\mathrm{YC}$ P3a/P3b amplitude $(p>.05)$ \\
\hline $\begin{array}{l}\text { Kimura et al. } \\
\text { (59) }\end{array}$ & 29 & $\begin{array}{l}\text { Trauma } \\
\text { (ND) }\end{array}$ & $\mathrm{N} / \mathrm{A}$ & $\mathrm{N} / \mathrm{A}$ & $\mathrm{P} ; \mathrm{TIME}$ & $Y(I E S-R)$ & $\begin{array}{l}\text { Higher trauma central P3a amplitude } \\
\text { linked to higher IES-R hyperarousal } \\
(p=.03, \rho=.40) \text { but not total }(p=.07) \text {, } \\
\text { intrusions }(p=.24) \text {, or avoidance }(p=.19) \text {. }\end{array}$ \\
\hline $\begin{array}{l}\text { Kimura et al. } \\
(60)\end{array}$ & 13 & $\begin{array}{l}\text { Trauma } \\
\text { (ND) }\end{array}$ & $\mathrm{N} / \mathrm{A}$ & $\mathrm{N} / \mathrm{A}$ & $\mathrm{N} / \mathrm{A}$ & $Y(I E S-R)$ & $\begin{array}{l}\text { No links between P3a amplitude and } \\
\text { IES-R total, intrusions, avoidance, or } \\
\text { hyperarousal (all } p \geq .30 \text { ); lower P3b } \\
\text { amplitude associated with higher IES-R } \\
\text { intrusions ( } p<.05, \rho=-.56 \text { ) but not any } \\
\text { other IES-R scores (all } p \geq .13 \text { ). }\end{array}$ \\
\hline
\end{tabular}
range: .14-.20) 
TABLE 4 | Continued

\begin{tabular}{|c|c|c|c|c|c|c|c|}
\hline Author (Ref.) & $\begin{array}{l}\text { Trauma } \\
\text { group } \\
\text { (T) }\end{array}$ & $\begin{array}{l}\text { Trauma } \\
\text { type }\end{array}$ & PTSD DX & $\begin{array}{l}\text { Control group } \\
\text { (C) trauma } \\
\text { exposure? }\end{array}$ & Clinical controls & $\begin{array}{l}\text { Symptom } \\
\text { correlations }\end{array}$ & Results \\
\hline $\begin{array}{l}\text { Lamprecht } \\
\text { et al. }(112)^{\star}\end{array}$ & 10 & $\begin{array}{l}\text { PTSD } \\
\text { (VAR) }\end{array}$ & DSM-IV; IES & $10 \mathrm{NC}$ & MED; SUD; TIME & $Y($ IES) & $\begin{array}{l}\mathrm{T}>\mathrm{NC} \text { for } \mathrm{N} 1 \text { amplitude }(p<.02) ; \mathrm{T}= \\
\mathrm{NC} \text { for } \mathrm{P} 2(p=.33), \mathrm{N} 2(p=.27) \text {, and } \\
\text { P3b ( } p=.58) \text { amplitudes; } \mathrm{T} \text { showed } \\
\text { novel P3a amplitude reduction post- as } \\
\text { compared to pre-treatment }(p<.03)\end{array}$ \\
\hline $\begin{array}{l}\text { Lewine et al. } \\
(51)^{\star}\end{array}$ & 31 & $\begin{array}{l}\text { PTSD } \\
\text { (COMBAT) }\end{array}$ & $\begin{array}{l}\text { SCID; CAPS; } \\
\text { M-PTSD }\end{array}$ & $\begin{array}{l}38 \mathrm{NC} ; 10 \\
\mathrm{MDD}+; 8 \text { AUD+ }\end{array}$ & $\mathrm{M} ; \mathrm{N}$ & $\begin{array}{l}\text { Y (CAPS; } \\
\text { M-PTSD; } \\
\text { HAM-D) }\end{array}$ & $\begin{array}{l}\mathrm{T}=\mathrm{NC} \text { for } \mathrm{N} 1 \text { amplitude }(p=.26) ; \\
\mathrm{NC}>\mathrm{T} \mathrm{P} 2 \text { amplitude to high intensity } \\
\text { tones }(p<.01 \text {, no effect size able to be } \\
\text { computed); within T, higher amplitude } \\
\text { linked to higher CAPs/M-PTSD (both } \\
\left.p=.03, R^{2}=.52\right) \text { and higher HAM-D } \\
\left(p=.04, R^{2}=.49\right) \text { scores }\end{array}$ \\
\hline $\begin{array}{l}\text { McFarlane } \\
\text { et al. }(35)^{\star}\end{array}$ & 214 & $\begin{array}{l}\text { Trauma } \\
\text { (ELS) }\end{array}$ & $\mathrm{N} / \mathrm{A}$ & 193 NC & M; N; P; SEX & $Y(E L S Q)$ & $\begin{array}{l}\text { NC }>\text { T for frontal N2 target amplitude } \\
(p=.006)\end{array}$ \\
\hline $\begin{array}{l}\text { McPherson } \\
\text { et al. }(113)^{\star}\end{array}$ & 60 & $\begin{array}{l}\text { PTSD } \\
\text { (CPA, } \\
\text { CSA) }\end{array}$ & DICA & 81 YC & SEX & $Y(D I C A)$ & $\begin{array}{l}\mathrm{T}>\text { YC P2-N2 amplitude to high } \\
\text { intensity tones }(p<.05) \text {; P2-N2 } \\
\text { amplitude linked to re-experiencing } \\
\text { symptoms }(p=.02)\end{array}$ \\
\hline $\begin{array}{l}\text { Menning } \\
\text { et al. }(114)^{*}\end{array}$ & 10 & $\begin{array}{l}\text { PTSD } \\
\text { (VAR) }\end{array}$ & SCID & $14 \mathrm{NC}$ & TIME & $\mathrm{N}$ & $\begin{array}{l}\mathrm{T}>\text { NC MMN amplitude }(p \leq .05) ; \mathrm{T}= \\
\text { NC for N1-P2 amplitude }(p=.22)\end{array}$ \\
\hline $\begin{array}{l}\text { Metzger et al. } \\
\text { (57) }\end{array}$ & 34 & $\begin{array}{l}\text { PTSD } \\
\text { (COMBAT, } \\
\text { CSA) }\end{array}$ & UNKN & $18 Y C$ & PANIC; SEX & $\mathrm{N}$ & $\begin{array}{l}Y C>T \text { target } \mathrm{P} 3 \mathrm{~b} \text { amplitudes }(p<.05 \\
d=1.30) \text {; in women, } \mathrm{YC}>\mathrm{T} \text { distractor } \\
\text { P3a amplitudes }(p=.04, d=.91)\end{array}$ \\
\hline $\begin{array}{l}\text { Metzger } \\
\text { et al. (49) }\end{array}$ & 29 & $\begin{array}{l}\text { PTSD } \\
\text { (COMBAT) }\end{array}$ & $\begin{array}{l}\text { SCID; CAPS; } \\
\text { PCL-M; } \\
\text { M-PTSD; } \\
\text { IES-R }\end{array}$ & 37 YC & PSY; MED & $\begin{array}{l}\text { Y (CAPS; } \\
\text { PCL-M) }\end{array}$ & $\begin{array}{l}\text { T > YC target P3b amplitude }(p<.05, \\
d=.50) ; \mathrm{T}>\mathrm{YC} \mathrm{P} 2 \text { target amplitude } \\
(p=.007, d=.68) ; \mathrm{T}>\mathrm{YC} \mathrm{P} 2 \text { frontal and } \\
\text { parietal non-target amplitude }(p=.03, \\
d=.46 \text { and } .54) ; \mathrm{T}>\mathrm{YC} \mathrm{P} 2 \text { target } \\
\text { latency }(p=.03, d=.55) ; \mathrm{T}>\mathrm{YC} \mathrm{P} 2 \\
\text { non-target latency }(p<.001, d=.91) ; \\
\mathrm{YC}>\mathrm{T} \text { frontal } \mathrm{N} 2 \text { target amplitude }(p= \\
.03, d=.52) ; \text { higher } \mathrm{P} 2 \text { amplitude linked } \\
\text { to greater CAPs total, re-experiencing, } \\
\text { avoidance/numbing, and arousal }(p \leq \\
.05 ; R^{2} \text { range: .08-.10) and IES-R }(p= \\
\left..05, R^{2}=.08\right) .\end{array}$ \\
\hline $\begin{array}{l}\text { Metzger et al. } \\
(52)\end{array}$ & 37 & $\begin{array}{l}\text { PTSD } \\
\text { (COMBAT) }\end{array}$ & $\begin{array}{l}\text { CAPS; } \\
\text { M-PTSD; } \\
\text { CSS }\end{array}$ & $\begin{array}{l}37 \mathrm{MZ} \text { twins NC; } \\
47 \mathrm{MZ} \text { twins } \mathrm{YC} \\
48 \mathrm{MZ} \text { twins NC }\end{array}$ & PSY; MED & $\mathrm{N}$ & $\begin{array}{l}\mathrm{T}>\mathrm{YC} / \mathrm{NC} \text { N2 latency }(p=.04, d=.45) \\
\mathrm{T}=\mathrm{YC} / \mathrm{NC} \text { for } \mathrm{P} 3 \mathrm{~b} \text { amplitude }(p>.05)\end{array}$ \\
\hline $\begin{array}{l}\text { Morgan \& } \\
\text { Grillon (62)* }\end{array}$ & 13 & $\begin{array}{l}\text { PTSD } \\
\text { (ASSAULT) }\end{array}$ & SCID & $16 \mathrm{NC}$ & M; MED; SUD & $\begin{array}{l}\text { Y (M-PTSD; } \\
\text { STAI) }\end{array}$ & $\begin{array}{l}\text { T }>\text { NC MMN target amplitude }(p< \\
.05) \text { and N2 target amplitude }(p<.02) \text {; } \\
\text { greater frontal MMN amplitude linked to } \\
\text { greater PTSD severity }\left(p<.05, R^{2}=.36\right)\end{array}$ \\
\hline $\begin{array}{l}\text { Neylan et al. } \\
(115)\end{array}$ & 25 & $\begin{array}{l}\text { PTSD } \\
\text { (COMBAT) }\end{array}$ & CAPS; SCID & $15 Y C$ & N; PANIC; PSY & Y & $\begin{array}{l}\mathrm{T}=\mathrm{YC} \text { P3a/P3b amplitude }(p>.27) \text { and } \\
\text { latency }(p>.05)\end{array}$ \\
\hline \multicolumn{8}{|c|}{ Auditory inhibition } \\
\hline $\begin{array}{l}\text { Schaefer \& } \\
\text { Nooner (63) }\end{array}$ & 12 & $\begin{array}{l}\text { Trauma } \\
\text { (VAR) }\end{array}$ & $\mathrm{N} / \mathrm{A}$ & $26 \mathrm{NC}$ & $\mathrm{N} / \mathrm{A}$ & $\begin{array}{l}\text { Y (TSC-40) and } \\
\text { depression }\end{array}$ & $\begin{array}{l}\mathrm{T}>\mathrm{NC} \text { frontal Go P3 latency (trauma }+ \\
\text { depression) ( } p=.003, d=1.14) ; \mathrm{NC}>\mathrm{T} \\
\text { central/parietal Go P3 latency (trauma }+ \\
\text { depression) ( } p<.02, d=.74 \text { and.81) }\end{array}$ \\
\hline
\end{tabular}

*Effect sizes for at least one significant effect unable to be computed based on article information. ACCID, accidents; ASD, acute stress disorder; AUD, alcohol use disorder; CAPS, Clinician Administered PTSD Scale; CPA, childhood physical abuse; CSA, childhood sexual abuse; CES, Combat Exposure Scale; CSS, Combat Severity Scale; DTS, Davidson Trauma Scale; DEP, excluded for depression; DICA, Diagnostic Interview for Children and Adolescents; DIS, Diagnostic Interview Schedule; DX, diagnosis; ELS, early life stress; ELSQ, Early Life Stress Questionnaire; HAM-D, Hamilton Depression Inventory; IES-R, Impact of Events Scale Revised; M, excluded for medical problems; M-PTSD, Mississippi Scale for Combat-Related PTSD; MDD, major depressive disorder; MED, excluded for medication; MMN, mismatch negativity event related potential; MZ, monozygotic; N, excluded for neurological problems; N/A, not applicable; N2, N200 event related potential; NC, trauma-no control group; ND, natural disaster; P, excluded for psychiatric diagnoses; P2, P200 event related potential; P3, P300 event related potential; P3a, frontocentral P300; P3b, centroparietal P300; PANIC, excluded for panic disorder; PCL-C, PTSD Checklist, Civilian Version; PCL-M, PTSD Checklist, Military Version; PSY, excluded for psychosis; SADS, Schedule for Affective Disorders and Schizophrenia for School-Age Children; SCID, Structured Clinical Interview for DSM-IV; SEX, explicitly examined sex differences; STAI, State Trait Anxiety Inventory; SUIC, excluded for suicidal ideation; SUD, excluded for substance use disorders; T, trauma group; TIME, reported time since trauma; TSC-40, Trauma Symptom Checklist 40; TERROR, terrorist attack; UNKN, unknown; VAR, various traumas; YC, trauma-yes control group. 
TABLE 5 | Visual event related potential (ERP) studies of trauma and/or posttraumatic stress disorder (PTSD).

\begin{tabular}{|c|c|c|c|c|c|c|c|}
\hline Author (Ref.) & $\begin{array}{l}\text { Trauma } \\
\text { group } \\
\text { (T) }\end{array}$ & $\begin{array}{l}\text { Trauma } \\
\text { type }\end{array}$ & PTSD DX & $\begin{array}{l}\text { Control group } \\
\text { (C) trauma } \\
\text { exposure? }\end{array}$ & $\begin{array}{l}\text { Clinical } \\
\text { controls }\end{array}$ & $\begin{array}{l}\text { Symptom } \\
\text { correlations }\end{array}$ & Results \\
\hline \multicolumn{8}{|c|}{ Visual attention and working memory } \\
\hline $\begin{array}{l}\text { Attias et al. } \\
(116)^{\star}\end{array}$ & 20 & $\begin{array}{l}\text { PTSD } \\
\text { (COMBAT) }\end{array}$ & UNKN & 20 YC & MED; N; P & $\mathrm{N}$ & $\begin{array}{l}\text { T > YC combat P3a amplitudes }(p<.05) ; \mathrm{T}>\text { YC } \\
\text { target/combat N2 amplitudes }(p<.01) ; \mathrm{T}>\text { YC P3 } \\
\text { target latency }(p<.02) \text {; no group differences in P2 } \\
\text { amplitudes }\end{array}$ \\
\hline $\begin{array}{l}\text { Bleich et al. } \\
(72)\end{array}$ & 20 & $\begin{array}{l}\text { PTSD } \\
\text { (COMBAT) }\end{array}$ & UNKN & $20 Y C$ & MED; N; P; & $\mathrm{N}$ & $\begin{array}{l}\text { T > YC target, combat, and irrelevant non-target } \\
\text { P3a/P3b amplitude }(p<.001 ; d=1.14)\end{array}$ \\
\hline Chu et al. (83) & 489 & $\begin{array}{l}\text { Trauma } \\
\text { (CHILD) }\end{array}$ & $\mathrm{N} / \mathrm{A}$ & $\mathrm{N} / \mathrm{A}$ & M; P; SEX & Y (ELSQ) & $\begin{array}{l}\text { N170 amplitude for angry }>\text { happy faces linked } \\
\text { to child trauma in right temporal region, such } \\
\text { that high trauma was associated with less N170 } \\
\text { differentiation between these faces than low } \\
\text { trauma ( } p<.04 \text {, partial } \eta^{2}=2.2 \% \text { variance); N170 } \\
\text { amplitude for angry }>\text { happy faces linked to adult } \\
\text { trauma in left temporal region, wherein high T was } \\
\text { associated with less N170 differentiation between } \\
\text { these faces than low trauma ( } p<.01 \text {, partial } \eta^{2}= \\
2.1 \% \text { variance) }\end{array}$ \\
\hline Chu et al. (85) & 72 & $\begin{array}{l}\text { Trauma/ } \\
\text { PTSD } \\
\text { (CHILD) }\end{array}$ & $\begin{array}{l}\text { CAPS; } \\
\mathrm{MINI}\end{array}$ & $\mathrm{N} / \mathrm{A}$ & $\begin{array}{l}\text { PSY; SEX; } \\
\text { SUD }\end{array}$ & $\begin{array}{l}\text { Y (CAPS; } \\
\text { ELSQ) }\end{array}$ & $\begin{array}{l}\text { Lower } \mathrm{N} 170 \text { amplitudes for fear }>\text { happy faces } \\
\text { linked to higher child trauma in left temporal } \\
\text { region }\left(p=.002 ; R^{2} \text { change }=.15\right) \text {; higher PTSD } \\
\text { avoidance linked to lower N170 amplitude to happy } \\
\left.\text { faces ( } p=.004 \text {, partial } \eta^{2}=.30\right) \text {. }\end{array}$ \\
\hline $\begin{array}{l}\text { DiGangi et al. } \\
(78)\end{array}$ & 73 & $\begin{array}{l}\text { Trauma } \\
\text { (VET) }\end{array}$ & $\begin{array}{l}\text { PCL-M; } \\
\text { MINI }\end{array}$ & N/A & $\begin{array}{l}\text { M; PSY; } \\
\text { SUIC }\end{array}$ & Y (PCL-M) & $\begin{array}{l}\text { Higher PTSD symptoms linked to smaller LPP } \\
\text { amplitude to angry faces }\left(p=.04 . R^{2}=.09\right) \text {, but not } \\
\text { happy or fear faces }(p>.05)\end{array}$ \\
\hline $\begin{array}{l}\text { DiGangi et al. } \\
\text { (79) }\end{array}$ & 47 & $\begin{array}{l}\text { Trauma } \\
\text { (VET) }\end{array}$ & $\begin{array}{l}\text { CAPS; } \\
\text { MINI; }\end{array}$ & $\mathrm{N} / \mathrm{A}$ & $\begin{array}{l}\text { M; PSY; } \\
\text { SUIC }\end{array}$ & Y (CAPS) & $\begin{array}{l}\text { Within T with greater perseverative errors, higher } \\
\text { PTSD symptoms linked to larger angry face LPP } \\
\text { amplitude, but in those with smaller errors, higher } \\
\text { PTSD symptoms linked to smaller LPP amplitude to } \\
\text { angry faces }\left(p=.02, R^{2}=.07\right)\end{array}$ \\
\hline $\begin{array}{l}\text { Duan et al. } \\
\text { (82) }\end{array}$ & 28 & $\begin{array}{l}\text { PTSD } \\
(N D)\end{array}$ & $\mathrm{PCL}$ & 30 YC & $\begin{array}{l}\text { MED; N; } \\
\text { SUD; TIME }\end{array}$ & $\mathrm{Y}(\mathrm{PCL})$ & $\begin{array}{l}\text { T > YC CNV amplitude }(p<.05, d=2.94) \text {; greater } \\
\text { CNV amplitude linked to higher PCL total }(p<.05 \\
\left.R^{2}=.07\right) \text { and re-experiencing }\left(p<.05, R^{2}=.09\right)\end{array}$ \\
\hline $\begin{array}{l}\text { Ehlers et al. } \\
(99)^{\star}\end{array}$ & 19 & $\begin{array}{l}\text { PTSD } \\
\text { (VAR) }\end{array}$ & SSAGA & 39 YC & $\mathrm{N} / \mathrm{A}$ & $\mathrm{N}$ & $\begin{array}{l}\text { T }>\text { YC happy central }(p<.04) \text { and right frontal }(p< \\
\text {.05) P3 latency }\end{array}$ \\
\hline $\begin{array}{l}\text { Gilmore et al. } \\
(70)\end{array}$ & $\begin{array}{l}33 \text { PTSD, } \\
19 \text { TBI, } \\
41 \text { TBI-PTSD }\end{array}$ & $\begin{array}{l}\text { PTSD } \\
\text { (COMBAT) }\end{array}$ & $\begin{array}{l}\text { CAPS; } \\
\text { SCID }\end{array}$ & $31 \mathrm{TBI}-\mathrm{YC}$ & $\begin{array}{l}\text { DEP; M; } \\
\text { PSY; SUD }\end{array}$ & Y (CAPS) & $\begin{array}{l}\text { No group differences in P1, N1, P2, or N2 } \\
\text { amplitudes (all } p>.05) \text {; YC }>\text { T parietal P3b target } \\
\text { amplitude }\left(p<.002 \text {, partial } \eta^{2}=.12 \text { ); no significant }\right. \\
\text { correlations between CAPS total/subscales and } \\
\text { P3b amplitude (all } p>.12 \text { ) }\end{array}$ \\
\hline $\begin{array}{l}\text { Grasso \& } \\
\text { Simons (117) }\end{array}$ & 19 & $\begin{array}{l}\text { PTSD } \\
\text { (CHILD) }\end{array}$ & KSADS & $19 \mathrm{NC}$ & $\begin{array}{l}\text { PSY; SUD; } \\
\text { SUIC; } \\
\text { TIME }\end{array}$ & $\mathrm{N}$ & $\mathrm{T}=\mathrm{NC}$ LPP amplitude to negative pictures $(p>.05)$ \\
\hline $\begin{array}{l}\text { Honzel et al. } \\
(118)^{\star}\end{array}$ & 17 & $\begin{array}{l}\text { PTSD + } \\
\text { TBI } \\
(\text { COMBAT) }\end{array}$ & PCL-M & $16 \mathrm{YC}$ & $\mathrm{P}$ & $\mathrm{N}$ & $\begin{array}{l}\text { Single task: } T=Y C \text { on } P 3 \text { amplitude }(p>.05) \text {; dual } \\
\text { task: } Y C \text { old probes }>\text { P3 amplitude than new } \\
\text { probes, whereas T old probes = new probes for P3 } \\
\text { amplitude }(p=.001)\end{array}$ \\
\hline $\begin{array}{l}\text { Kessel et al. } \\
(119)\end{array}$ & 37 & $\begin{array}{l}\text { Trauma } \\
\text { (ND) }\end{array}$ & $\mathrm{N} / \mathrm{A}$ & 40 YC & M; TIME & $\begin{array}{l}Y \text { (hurricane } \\
\text { stress) }\end{array}$ & $\begin{array}{l}\text { YC lower post- than pre-trauma negative LPP } \\
\text { amplitude }(p=.007, d=.61) \text {; within T, negative LPP } \\
\text { amplitude did not change pre- and post-trauma } \\
(p=.93)\end{array}$ \\
\hline $\begin{array}{l}\text { Kimble et al. } \\
(120)^{*}\end{array}$ & 22 & $\begin{array}{l}\text { PTSD } \\
\text { (VAR) }\end{array}$ & $\begin{array}{l}\text { SCID; } \\
\text { CAPS }\end{array}$ & 35 YC & $\begin{array}{l}\text { N; PSY; } \\
\text { SUD }\end{array}$ & $\mathrm{N}$ & $\begin{array}{l}\text { YC }>\text { T N4 amplitude to threatening versus } \\
\text { expected sentence endings }(p<.01) ; Y C=T \text { for } \\
\text { N4 latency }(p>.05)\end{array}$ \\
\hline $\begin{array}{l}\text { Kimble et al. } \\
\text { (86) }\end{array}$ & 18 & $\begin{array}{l}\text { PTSD } \\
\text { (VAR) }\end{array}$ & PSS; TES & 21 YC & $\begin{array}{l}\text { N; MED; } \\
\text { SUD }\end{array}$ & Y (PTCl) & $\begin{array}{l}\text { T = YC N4 amplitude to positive, negative, and } \\
\text { incongruent sentence endings }\left(p>.05 ; \eta^{2}=.01\right) ; \\
\text { greater PTCl negative cognitions about the world } \\
\text { linked to larger N4 amplitudes to negative sentence } \\
\left.\text { endings ( } p<.01 ; R^{2}=.18\right)\end{array}$ \\
\hline $\begin{array}{l}\text { Klimova et al. } \\
(121)^{\star}\end{array}$ & 39 & $\begin{array}{l}\text { PTSD } \\
\text { (VAR) }\end{array}$ & CAPS & $\mathrm{N} / \mathrm{A}$ & $\begin{array}{l}\text { N; SUD; } \\
\text { TIME }\end{array}$ & Y (CADSS) & $\begin{array}{l}\text { Low dissociating } T>\text { high dissociating T P2 amplitude } \\
\text { for consciously presented happy faces }(p=.04)\end{array}$ \\
\hline
\end{tabular}


TABLE 5 | Continued

\begin{tabular}{|c|c|c|c|c|c|c|c|}
\hline Author (Ref.) & $\begin{array}{l}\text { Trauma } \\
\text { group } \\
\text { (T) }\end{array}$ & $\begin{array}{l}\text { Trauma } \\
\text { type }\end{array}$ & PTSD DX & $\begin{array}{l}\text { Control group } \\
\text { (C) trauma } \\
\text { exposure? }\end{array}$ & $\begin{array}{l}\text { Clinical } \\
\text { controls }\end{array}$ & $\begin{array}{l}\text { Symptom } \\
\text { correlations }\end{array}$ & Results \\
\hline $\begin{array}{l}\text { Kounios et al. } \\
(122)^{\star}\end{array}$ & 8 & $\begin{array}{l}\text { PTSD } \\
\text { (COMBAT) }\end{array}$ & UNKN & $8 Y C$ & N/A & $\mathrm{N}$ & $\begin{array}{l}\text { T > YC central P3 amplitude; T > YC LPP } \\
\text { amplitude (no } p \text { values provided) }\end{array}$ \\
\hline $\begin{array}{l}\text { Kujawa et al. } \\
(80)\end{array}$ & 260 & $\begin{array}{l}\text { Trauma } \\
\text { (ND) }\end{array}$ & $\mathrm{N} / \mathrm{A}$ & N/A & M; TIME & $\begin{array}{l}\text { Y (hurricane } \\
\text { stress; } \\
\text { CBCL) }\end{array}$ & $\begin{array}{l}\text { Higher stress interacted with greater LPP amplitude } \\
\text { to unpleasant images to predict greater externalizing } \\
\text { symptoms }\left(p<.001, R^{2} \text { change }=.07\right) \text {, whereas } \\
\text { higher stress and larger LPP amplitude to unpleasant } \\
\text { images ( } p \leq .04, R^{2} \text { change }=.05 \text { ) predicted higher } \\
\text { internalizing symptoms }\end{array}$ \\
\hline $\begin{array}{l}\text { Lobo et al. } \\
\text { (81) }\end{array}$ & 43 & $\begin{array}{l}\text { Trauma } \\
\text { (VAR) }\end{array}$ & PCL-C & $\mathrm{N} / \mathrm{A}$ & MED; N; P; & Y (PCL-C) & $\begin{array}{l}\text { Higher trauma symptoms linked to greater unpleasant } \\
\text { minus neutral LPP amplitude }\left(p<.01, R^{2}=.22\right)\end{array}$ \\
\hline $\begin{array}{l}\text { MacNamara } \\
\text { et al. (66) }\end{array}$ & 19 & $\begin{array}{l}\text { PTSD } \\
\text { (COMBAT) }\end{array}$ & $\begin{array}{l}\text { SCID; } \\
\text { CAPS; } \\
\text { PCL-M; } \\
\text { CES }\end{array}$ & $14 Y C$ & $\begin{array}{l}\text { M; MED; } \\
\text { N; PSY }\end{array}$ & Y (CAPS) & $\begin{array}{l}\text { YC }>\text { T P2 amplitudes for emotional faces }(p<.01 \text {; } \\
\left.\text { partial } \eta^{2}=.25\right) ; \text { YC }>\text { T LPP amplitudes to angry } \\
\text { faces }\left(p<.01 \text {, partial } \eta^{2}=.12\right) \text {; smaller LPP to } \\
\text { fearful faces linked to greater CAPs intrusions } \\
\left(p<.02, R^{2}=.31\right)\end{array}$ \\
\hline $\begin{array}{l}\text { Saar- } \\
\text { Ashkenazy } \\
\text { et al. (77) }\end{array}$ & 14 & $\begin{array}{l}\text { PTSD } \\
\text { (VAR) }\end{array}$ & CAPS & $14 \mathrm{NC}$ & $\mathrm{N} ; \mathrm{P}$ & $\mathrm{N}$ & $\begin{array}{l}\text { T }=\text { NC P300 amplitude }(p>.05) \text {; NC }>\text { T neutral } \\
\text { P600 amplitude }\left(p=.05, \text { partial } \eta^{2}=.22\right) \text {; a group } \\
\left.\text { x region } x \text { emotion interaction (partial } \eta^{2}=.16\right) \\
\text { showed that T }>\text { NC positive left frontocentral LPP } \\
\text { amplitude }(p<.01) \text { and T }>\text { NC neutral left parieto- } \\
\text { occipital LPP amplitude }(p<.05)\end{array}$ \\
\hline $\begin{array}{l}\text { Shah et al. } \\
(71)\end{array}$ & 18 & $\begin{array}{l}\text { PTSD } \\
\text { (VAR) }\end{array}$ & PCL; IES & $18 \mathrm{NC}$ & N; PSY & $\mathrm{N}$ & $\begin{array}{l}\text { NC > T P3 amplitude to neutral-neutral dot probe } \\
\text { condition }(p<.05, d=.71)\end{array}$ \\
\hline $\begin{array}{l}\text { Shu, Onton, } \\
\text { Prabhakar } \\
\text { et al. }(123)^{\star}\end{array}$ & $16+\mathrm{TBI}$ & $\begin{array}{l}\text { PTSD } \\
\text { (COMBAT) }\end{array}$ & $\begin{array}{l}\text { SCID; } \\
\text { CAPS }\end{array}$ & $16+$ TBI YC & $\begin{array}{l}\text { ADHD; M; } \\
\text { PSY; SUD }\end{array}$ & Y (CAPS) & $\begin{array}{l}\text { T > YC greater N170/P2, N2/P3, and LPP } \\
\text { amplitudes (all } p<.05) \text {; N2/P3 amplitude correlated } \\
\text { with CAPS total }(p<.01 \text { ) }\end{array}$ \\
\hline $\begin{array}{l}\text { Sokhadze } \\
\text { et al. }(124)^{\star}\end{array}$ & $10+C D$ & $\begin{array}{l}\text { PTSD } \\
(\text { UNKN) }\end{array}$ & $\begin{array}{l}\text { SCID; } \\
\text { PSS }\end{array}$ & $\begin{array}{l}12 \mathrm{CD}+\mathrm{NC} ; 9 \\
\mathrm{CD}-\mathrm{NC}\end{array}$ & $\mathrm{N} / \mathrm{A}$ & $\mathrm{N}$ & $\begin{array}{l}\text { T > CD-P3a amplitude to trauma }(p<.01) ; T> \\
\text { CD+ P3a trauma latency }(p=.01)\end{array}$ \\
\hline $\begin{array}{l}\text { Stanford } \\
\text { et al. (68) }\end{array}$ & 10 & $\begin{array}{l}\text { PTSD } \\
\text { (COMBAT) }\end{array}$ & $\begin{array}{l}\text { SCID; } \\
\text { PCL-M; } \\
\text { CES }\end{array}$ & $10 Y C$ & $\begin{array}{l}\text { ADHD; M; } \\
\text { N; PSY; } \\
\text { SUD }\end{array}$ & $\mathrm{N}$ & $\begin{array}{l}\text { YC > T right parietal target P3 amplitude }(p=.03, \\
d=.45) ; \mathrm{T}>\mathrm{YC} \text { frontal combat P3 amplitude }(p< \\
.05, d=.90) ; \mathrm{YC}>\mathrm{T} \text { P3 central and parietal target } \\
\text { latency }(p \leq .03, d=.56 \text { and.81). }\end{array}$ \\
\hline $\begin{array}{l}\text { Tillman et al. } \\
\text { (73) }\end{array}$ & 22 & $\begin{array}{l}\text { Trauma } \\
\text { (COMBAT) }\end{array}$ & $\begin{array}{l}\text { SCID; } \\
\text { CAPS; } \\
\text { M-PTSD }\end{array}$ & $8 Y C$ & $\mathrm{~N} / \mathrm{A}$ & Y (M-PTSD) & $\begin{array}{l}\text { T }=\text { YC for P3a amplitude }(p=.80) ; \mathrm{YC}>\mathrm{T} \text { target } \\
\text { P3b amplitude }\left(p=.001, \eta^{2}=.38\right) ; \text { lower target } \\
\text { P3b amplitude linked to higher hyperarousal } \\
\left(p=.004, R^{2}=.63\right)\end{array}$ \\
\hline $\begin{array}{l}\text { Trujillo et al. } \\
\text { (84) }\end{array}$ & 30 & $\begin{array}{l}\text { Trauma } \\
\text { (COMBAT) }\end{array}$ & $\mathrm{N} / \mathrm{A}$ & $20 \mathrm{NC}$ & $\begin{array}{l}\text { DEP; MED; } \\
\text { N; SUD }\end{array}$ & $\mathrm{N}$ & $\begin{array}{l}\text { T }>\text { NC N170 amplitude to faces than words } \\
(p<.001, d=.60)\end{array}$ \\
\hline Tso et al. (75) & 31 & $\begin{array}{l}\text { Trauma } \\
\text { (TERROR) }\end{array}$ & $\mathrm{N} / \mathrm{A}$ & $\mathrm{N} / \mathrm{A}$ & $\begin{array}{l}\text { M; MED; } \\
\text { P; TIME }\end{array}$ & $Y($ IES-R) & $\begin{array}{l}\text { Higher avoidance linked to lower P3 and LPP } \\
\text { amplitude to attack than neutral stimuli (both } \\
p<.01, R^{2}=.24 \text { and } .25 \text { ) }\end{array}$ \\
\hline $\begin{array}{l}\text { Veltmeyer et } \\
\text { al. (69) }\end{array}$ & 34 & $\begin{array}{l}\text { PTSD } \\
\text { (VAR) }\end{array}$ & $\begin{array}{l}\text { CAPS; } \\
\text { PCL }\end{array}$ & $136 \mathrm{NC}$ & $\begin{array}{l}\text { ANX; PSY; } \\
\text { SUD; TIME }\end{array}$ & Y (CAPS) & $\begin{array}{l}\mathrm{NC}>\mathrm{T} \text { left temporal and midline centroparietal P3 } \\
\text { amplitude (both } p<.01, d=.29 \text { and } .51) \text {; no links } \\
\text { between CAPS and ERPs }(p>.05)\end{array}$ \\
\hline $\begin{array}{l}\text { Wang et al. } \\
(74)\end{array}$ & 65 & $\begin{array}{l}\text { Trauma } \\
\text { (COMBAT) }\end{array}$ & $\begin{array}{l}\text { CAPS; } \\
\text { PCL-M }\end{array}$ & N/A & $\begin{array}{l}\text { N; PSY; } \\
\text { SUIC }\end{array}$ & $\mathrm{N}$ & $\begin{array}{l}\text { Lower target P3b amplitude predicted future PTSD } \\
\text { conversion ( } p<.05, d \text { range: } 1.11-1.36) \text {, but no } \\
\text { effect emerged for P3b latency }(p>.05)\end{array}$ \\
\hline $\begin{array}{l}\text { Wang et al. } \\
(76)\end{array}$ & 30 & $\begin{array}{l}\text { Trauma } \\
\text { (COMBAT) }\end{array}$ & $\begin{array}{l}\text { CAPS; } \\
\text { PCL-M }\end{array}$ & $\mathrm{N} / \mathrm{A}$ & DEP; N & $\begin{array}{l}\text { Y (CAPS; } \\
\text { PCL-M) }\end{array}$ & $\begin{array}{l}\text { No subjects had PTSD at baseline. Increased target } \\
\text { P3b amplitude }\left(p<.0001, R^{2}=.52\right) \text { and latency } \\
\left.\text { ( } p=.02, R^{2}=.18\right) \text { change was associated with } \\
\text { greater PTSD and depression symptom reductions } \\
\text { from baseline to follow-up }\end{array}$ \\
\hline $\begin{array}{l}\text { Wessa et al. } \\
(67)\end{array}$ & 7 & $\begin{array}{l}\text { PTSD } \\
\text { (ACCID) }\end{array}$ & $\begin{array}{l}\text { SCID; } \\
\text { CAPS }\end{array}$ & $7 \mathrm{YC} ; 7 \mathrm{NC}$ & $\mathrm{N}$ & Y (PDS) & $\begin{array}{l}\text { NC > T P2 amplitude for neutral }(d=1.19) \text {, positive } \\
(d=1.30) \text {, accident }(d=1.32) \text { pictures (all } p<.05) \text {; } \\
\text { YC }>\text { T P2 amplitude for neutral }(d=1.56) \text {, positive } \\
(d=1.43) \text {, accident }(d=2.85) \text { pictures (all } p<.05) \text {; } \\
\text { no LPP differences. }\end{array}$ \\
\hline $\begin{array}{l}\text { Wessa et al. } \\
(125)^{\star}\end{array}$ & 16 & $\begin{array}{l}\text { PTSD } \\
(\text { UNKN) }\end{array}$ & $\begin{array}{l}\text { SCID; } \\
\text { CAPS }\end{array}$ & 15 YC; 16 NC & $\mathrm{N}$ & $\mathrm{N}$ & $\begin{array}{l}\text { T }>\text { NC P3/LPP amplitude to trauma questions } \\
(p<.05) ; \text { NC }>\text { T and YC frontal CNV trauma } \\
\text { amplitude }(p \leq .04)\end{array}$ \\
\hline
\end{tabular}


TABLE 5 | Continued

\begin{tabular}{|c|c|c|c|c|c|c|c|}
\hline Author (Ref.) & $\begin{array}{l}\text { Trauma } \\
\text { group } \\
\text { (T) }\end{array}$ & $\begin{array}{l}\text { Trauma } \\
\text { type }\end{array}$ & PTSD DX & $\begin{array}{l}\text { Control group } \\
\text { (C) trauma } \\
\text { exposure? }\end{array}$ & $\begin{array}{l}\text { Clinical } \\
\text { controls }\end{array}$ & $\begin{array}{l}\text { Symptom } \\
\text { correlations }\end{array}$ & Results \\
\hline $\begin{array}{l}\text { Yun et al. } \\
(126)\end{array}$ & 12 & $\begin{array}{l}\text { Trauma } \\
\text { (ND) }\end{array}$ & PTSD-SS & 12 YC & $\mathrm{N} ; \mathrm{P}$ & $\mathrm{N}$ & $\begin{array}{l}\text { T: earthquake-related stimuli elicited larger P3 } \\
\text { amplitude than unrelated stimuli ( } p<.06, d=.74 \\
\text { within-group), pattern not seen in YC; P3 localized } \\
\text { to parahippocampal gyrus }\end{array}$ \\
\hline $\begin{array}{l}\text { Zhang et al. } \\
\text { (64) }\end{array}$ & 13 & $\begin{array}{l}\text { Trauma } \\
\text { (ND) }\end{array}$ & PTSD-SS & $13 \mathrm{NC}$ & $\mathrm{N} / \mathrm{A}$ & $\mathrm{N}$ & T > NC P2 amplitude $\left(p=.03\right.$, partial $\left.\eta^{2}=.17\right)$ \\
\hline $\begin{array}{l}\text { Zhang et al. } \\
(127)^{\star}\end{array}$ & 13 & $\begin{array}{l}\text { Trauma } \\
\text { (ND) }\end{array}$ & PTSD-SS & $13 \mathrm{NC}$ & $\mathrm{N} / \mathrm{A}$ & $\mathrm{N}$ & $\begin{array}{l}\text { NC }>\text { T P2 latency to trauma stimuli }(p<.01) ; T> \\
\text { NC P2 frontal/central amplitude to trauma stimuli } \\
(p<.05) ; T>\text { NC P3 amplitude to trauma stimuli } \\
(p<.001) ; T>\text { NC LPP amplitude to trauma stimuli } \\
(p<.05)\end{array}$ \\
\hline Zuj et al. (65) & 21 & $\begin{array}{l}\text { PTSD } \\
\text { (COMBAT) }\end{array}$ & PCL-M & $21 Y C$ & $\mathrm{~N}$ & $\mathrm{~N}$ & $\begin{array}{l}\text { T > YC P1 }\left(p=.02 \text {, partial } \eta^{2}=.13\right) \text { and P2 } \\
\left(p=.001, \text { partial } \eta^{2}=.13\right) \text { amplitude to angry faces } \\
\text { pre- to post-deployment; } \mathrm{T}=\mathrm{YC} \text { for } \mathrm{N} 170, \mathrm{~N} 2 \text {, and } \\
\text { P3 amplitude (all } p>.05)\end{array}$ \\
\hline \multicolumn{8}{|c|}{ Visual inhibition } \\
\hline $\begin{array}{l}\text { Chen et al. } \\
(128)^{\star}\end{array}$ & 11 & $\begin{array}{l}\text { PTSD } \\
\text { (ND) }\end{array}$ & PSS & 11 YC & $\begin{array}{l}\text { M; MED; } \\
\text { P; TIME }\end{array}$ & $\mathrm{N}$ & $\begin{array}{l}\text { YC > T P2 amplitude ( } p=.03) \text {; YC: greater P3 } \\
\text { amplitude for trauma than non-trauma }(p=.003) \\
\text { but T P3 conditions did not differ }(p>.05)\end{array}$ \\
\hline $\begin{array}{l}\text { Covey et al. } \\
(90)\end{array}$ & 14 & $\begin{array}{l}\text { Trauma } \\
\text { (POLICE) }\end{array}$ & $\begin{array}{l}\text { CAPS; } \\
\text { PCL-C }\end{array}$ & $11 \mathrm{NC}$ & M; MED; P & Y (CAPS) & $\begin{array}{l}\text { T }>\text { NC P3 amplitude across conditions ( } p<.01 \text {, } \\
\left.\text { partial } \eta^{2}=.26\right) ; T=\text { NC for P3 latency and N2 } \\
\text { nogo amplitude }(p>.05) \text {; higher frontal nogo P3 } \\
\text { amplitude linked to greater CAPS lifetime and total } \\
\left.\text { scores (all } p<.06, R^{2} \text { range: } .27-.48\right)\end{array}$ \\
\hline Cui et al. (87) & 19 & $\begin{array}{l}\text { PTSD } \\
\text { (VIOLENT) }\end{array}$ & PCL-C & $15 \mathrm{NC}$ & $M ; P$ & $\mathrm{~N}$ & $\begin{array}{l}\text { NC > T P2 amplitude across conditions }(p=.02 \\
d=.66)\end{array}$ \\
\hline $\begin{array}{l}\text { Gorka et al. } \\
(129)\end{array}$ & 43 & $\begin{array}{l}\text { PTSD } \\
\text { (VET) }\end{array}$ & $\begin{array}{l}\text { CAPS; } \\
\text { SCID }\end{array}$ & $24 Y C$ & M; N; PSY & $\begin{array}{l}Y(C E S, \\
\text { CAPS) }\end{array}$ & $\begin{array}{l}\mathrm{T}=\mathrm{YC} \text { in ERN amplitude }(p>.05) \text {; within T, AUD+ } \\
\text { had greater ERN amplitudes than PTSD alone } \\
(p<.01 \text {, Hedge's } g=.34) \text {; CES and CAPS were } \\
\text { unrelated to ERN amplitude }(p>.05)\end{array}$ \\
\hline $\begin{array}{l}\text { Khan et al. } \\
\text { (93) }\end{array}$ & 67 & $\begin{array}{l}\text { Trauma } \\
\text { (VET) }\end{array}$ & $\begin{array}{l}\text { MINI; } \\
\text { CAPS; } \\
\text { CES }\end{array}$ & $\mathrm{N} / \mathrm{A}$ & $\begin{array}{l}\text { M; N; PSY; } \\
\text { SUD }\end{array}$ & $\begin{array}{l}\text { Y (CES; } \\
\text { DRRI-2) }\end{array}$ & $\begin{array}{l}\text { Greater combat exposure (DRRI-2) linked to } \\
\text { larger ERN amplitude above and beyond anxiety } \\
\text { and PTSD symptoms }\left(p=.01, R^{2}=.10\right) \text {; no } \\
\text { relationship between ERN amplitude and CES } \\
\left(p=.26, R^{2}=.02\right) .\end{array}$ \\
\hline $\begin{array}{l}\text { Lackner et al. } \\
(130)^{\star}\end{array}$ & 43 & $\begin{array}{l}\text { Trauma } \\
\text { (CHILD) }\end{array}$ & CTES & $\mathrm{N} / \mathrm{A}$ & $\mathrm{N} / \mathrm{A}$ & Y (CTES) & $\begin{array}{l}\text { High } T \text { showed larger correct related negativity } \\
(\mathrm{CRN}) \text { minus } E R N \text { difference compared to low and } \\
\text { medium } T(p=.10)\end{array}$ \\
\hline $\begin{array}{l}\text { Lieberman } \\
\text { et al. }(131)^{\star}\end{array}$ & 47 & $\begin{array}{l}\text { Trauma } \\
\text { (VAR) }\end{array}$ & SCID & $\mathrm{N} / \mathrm{A}$ & $\begin{array}{l}\text { MED; N; } \\
\text { PSY; SEX }\end{array}$ & Y (SCID) & $\begin{array}{l}\text { Higher ERN amplitude linked to greater PTSD } \\
\text { hyperarousal }(p=.03) \text { and avoidance }(p=.06) \\
\text { symptoms }\end{array}$ \\
\hline $\begin{array}{l}\text { Melara et al. } \\
(95)\end{array}$ & 16 & PTSD & $\begin{array}{l}\text { SCID; } \\
\text { CAPS }\end{array}$ & $14 \mathrm{YC} ; 15 \mathrm{NC}$ & $\begin{array}{l}\text { DEP; PSY; } \\
\text { SUD; SUIC }\end{array}$ & & $\begin{array}{l}\text { T }>\text { YC/NC threat-related distractor frontocentral } \\
\text { positivity } 600-900 \mathrm{~ms}\left(p<.05, \eta^{2}=.05\right) \text { localized } \\
\text { to posterior cingulate cortex }\end{array}$ \\
\hline $\begin{array}{l}\text { Metzger et al. } \\
(57)\end{array}$ & 9 & $\begin{array}{l}\text { PTSD } \\
\text { (VAR) }\end{array}$ & SCID & $10 \mathrm{NC}$ & MED & $\mathrm{N}$ & $\begin{array}{l}\text { T }>\text { NC P3 latency across conditions }(p=.03, d= \\
1.00) ; \text { NC }>\text { T P3 amplitude across conditions } \\
(p=.0002, d=1.54)\end{array}$ \\
\hline $\begin{array}{l}\text { Meyer et al. } \\
(94)\end{array}$ & 223 & $\begin{array}{l}\text { Trauma } \\
\text { (ND) }\end{array}$ & $\mathrm{N} / \mathrm{A}$ & $\mathrm{N} / \mathrm{A}$ & $M$ & $\begin{array}{l}\text { Y (hurricane } \\
\text { trauma; } \\
\text { CBCL) }\end{array}$ & $\begin{array}{l}\text { Children with higher stress and higher ERN } \\
\text { amplitude show greater internalizing symptoms } \\
\text { post-trauma }\left(p<.01, R^{2}=.06\right)\end{array}$ \\
\hline $\begin{array}{l}\text { Rabinak et al. } \\
\text { (132) }\end{array}$ & 16 & $\begin{array}{l}\text { PTSD } \\
\text { (COMBAT) }\end{array}$ & $\begin{array}{l}\text { SCID; } \\
\text { CAPS; } \\
\text { PCL-M; } \\
\text { CES }\end{array}$ & 18 YC; 16 NC & MED & $N$ & $\mathrm{~T}=\mathrm{YC} / \mathrm{NC}$ for ERN amplitude $(p>.05)$ \\
\hline $\begin{array}{l}\text { Qiu et al. } \\
\text { (133) }\end{array}$ & 12 & $\begin{array}{l}\text { Trauma } \\
\text { (ND) }\end{array}$ & $\mathrm{N} / \mathrm{A}$ & 12 YC & $\mathrm{N} ; \mathrm{P}$ & $\mathrm{N}$ & $\begin{array}{l}\text { Within T, N400/600 to incongruent than congruent } \\
(p<.05 \text {, within-group } d=.57) \text {, localized to right } \\
\text { prefrontal regions }\end{array}$ \\
\hline
\end{tabular}


TABLE 5 | Continued

\begin{tabular}{|c|c|c|c|c|c|c|c|}
\hline Author (Ref.) & $\begin{array}{l}\text { Trauma } \\
\text { group } \\
\text { (T) }\end{array}$ & $\begin{array}{l}\text { Trauma } \\
\text { type }\end{array}$ & PTSD DX & $\begin{array}{l}\text { Control group } \\
\text { (C) trauma } \\
\text { exposure? }\end{array}$ & $\begin{array}{l}\text { Clinical } \\
\text { controls }\end{array}$ & $\begin{array}{l}\text { Symptom } \\
\text { correlations }\end{array}$ & Results \\
\hline $\begin{array}{l}\text { Shucard et al. } \\
(91)^{\star}\end{array}$ & 23 & $\begin{array}{l}\text { PTSD } \\
\text { (COMBAT) }\end{array}$ & $\begin{array}{l}\text { CAPS; } \\
\text { DTS; } \\
\text { SCID }\end{array}$ & $13 \mathrm{NC}$ & N; SUD & Y (CAPS) & $\begin{array}{l}T=N C \text { for go/nogo P3 amplitude }(p>.05) ; \\
T>N C \text { nogo P3 latency }(p=.001, d=1.07) ; \\
T>N C \text { distractor frontal P3 amplitude }(p=.001 \text {; } \\
\text { effect size not able to be calculated); longer } \\
\text { P3 go and nogo latency linked to higher CAPS } \\
\left.\text { hyperarousal (both } p<.05, R^{2}=.19, .21\right) \text {; longer } \\
\text { frontal, central, and parietal distractor latency } \\
\text { linked to greater re-experiencing (all } p<.01, R^{2} \\
\text { range }=.31-.46)\end{array}$ \\
\hline $\begin{array}{l}\text { Shu, Onton, } \\
\text { O'Connell } \\
\text { et al. }(134)^{\star}\end{array}$ & $17+\mathrm{TBl}$ & $\begin{array}{l}\text { PTSD } \\
\text { (COMBAT) }\end{array}$ & $\begin{array}{l}\text { SCID; } \\
\text { CAPS }\end{array}$ & $15+\mathrm{TBI} Y \mathrm{YC}$ & $\begin{array}{l}\text { ADHD; M; } \\
\text { PSY; SUD }\end{array}$ & Y (CAPS) & $\begin{array}{l}\text { T > YC N2 amplitude ( } p<.001) \text {; no group } \\
\text { differences in P3 amplitude }(p>.05) \text {; greater N2 } \\
\text { amplitude linked to worse CAPS total, avoidance/ } \\
\text { numbing, and hyperarousal }(p<.001) \text {; N2 localized } \\
\text { to dorsal anterior cingulate }\end{array}$ \\
\hline $\begin{array}{l}\text { Swick et al. } \\
(135)\end{array}$ & $14+$ TBI & $\begin{array}{l}\text { PTSD } \\
\text { (COMBAT) }\end{array}$ & $\begin{array}{l}\text { SCID; } \\
\text { PCL-M }\end{array}$ & 5 YC; 9 NC & SUD & $\begin{array}{l}\text { Y (PCL-M, } \\
\mathrm{BDI})\end{array}$ & $\begin{array}{l}\mathrm{T}=\mathrm{YC} / \mathrm{NC} \text { on } \mathrm{ERN} \text { amplitude }(p>.05) \text {; ERN not } \\
\text { correlated with PTSD or depression symptoms } \\
\text { within } \mathrm{T} \text { (all } p \geq .25 \text { ) }\end{array}$ \\
\hline Wei et al. (88) & 14 & $\begin{array}{l}\text { Trauma } \\
\text { (ND) }\end{array}$ & PTSD-SS & $14 \mathrm{NC}$ & N; P; TIME & $N$ & $\begin{array}{l}\text { T: P2 and P3 amplitudes greater for positive than } \\
\text { negative words (both } p<.05 \text {, within-group } d= \\
1.14 \text { and 1.83), latter localized to parahippocampal } \\
\text { gyrus/cuneus }\end{array}$ \\
\hline Wu et al. (89) & 16 & $\begin{array}{l}\text { PTSD } \\
\text { (ND) }\end{array}$ & PCL-C & $9 Y C$ & $\begin{array}{l}\text { MED; N; P; } \\
\text { SUD }\end{array}$ & $\mathrm{N}$ & $\begin{array}{l}\text { YC }>\text { T nogo N2 latency }(p=.02, d=1.03) ; Y C=T \\
\text { P3 amplitude/latency }(p>.05)\end{array}$ \\
\hline Wu et al. (92) & 54 & $\begin{array}{l}\text { Trauma } \\
\text { (ND) }\end{array}$ & PCL-S & N/A & $\begin{array}{l}\text { MED; N; P; } \\
\text { SUD }\end{array}$ & Y (PCL-S) & $\begin{array}{l}\text { Greater PTSD avoidance symptoms linked to } \\
\text { longer P3 nogo latency }\left(p=.01, R^{2}=.13\right)\end{array}$ \\
\hline \multicolumn{8}{|c|}{ Emotion regulation and reward processing } \\
\hline $\begin{array}{l}\text { Fitzgerald } \\
\text { et al. (96) }\end{array}$ & 25 & $\begin{array}{l}\text { PTSD } \\
\text { (COMBAT) }\end{array}$ & $\begin{array}{l}\text { SCID; } \\
\text { MINI; } \\
\text { CAPS; } \\
\text { PCL-M; } \\
\text { CES }\end{array}$ & 25 YC & M; N; PSY & $\mathrm{N}$ & $\begin{array}{l}T=Y C \text { for LPP before or during reappraisal } \\
(p>.05) ; T \text { showed lower LPP increases than YC } \\
\text { for maintain }\left(p=.02 \text {, partial } \eta^{2}=.09\right)\end{array}$ \\
\hline $\begin{array}{l}\text { Fitzgerald } \\
\text { et al. }(136)^{\star}\end{array}$ & 54 & $\begin{array}{l}\text { Trauma/ } \\
\text { PTSD } \\
\text { (COMBAT) }\end{array}$ & $\begin{array}{l}\text { MINI; } \\
\text { CAPS; } \\
\text { CES }\end{array}$ & N/A & $\begin{array}{l}\text { M; N; PSY; } \\
\text { SUIC }\end{array}$ & Y (CAPS) & $\begin{array}{l}\text { Smaller change in LPP amplitude during reappraisal } \\
\text { was associated with greater PTSD re-experiencing } \\
(p<.01) \text { and avoidance }(p=.03) \text {; smaller change } \\
\text { in LPP amplitude during emotion experience was } \\
\text { linked to lower PTSD avoidance symptoms over } \\
\text { time }(p<.01)\end{array}$ \\
\hline Li et al. $(137)^{\star}$ & 18 & $\begin{array}{l}\text { Trauma } \\
\text { (ND) }\end{array}$ & $\mathrm{N} / \mathrm{A}$ & $22 \mathrm{NC}$ & TIME & $\mathrm{N}$ & T > NC for P2 and P3 amplitudes (both $p<.05)$ \\
\hline $\begin{array}{l}\text { Pechtel et al. } \\
(138)^{\star}\end{array}$ & $\begin{array}{l}15 \text { past } \\
\text { MDD+ }\end{array}$ & $\begin{array}{l}\text { Trauma } \\
\text { (CSA) }\end{array}$ & $\mathrm{N} / \mathrm{A}$ & $\begin{array}{l}\text { DC: } 16 \text { past } \\
\text { MDD+; NC: } 18 \\
\text { past MDD- }\end{array}$ & $\begin{array}{l}\text { M; MED; } \\
\text { N; PSY; } \\
\text { SUD }\end{array}$ & $N$ & $\begin{array}{l}\mathrm{NC}=\mathrm{T} \text { ERN amplitude }(p=.10) ; \mathrm{T}>\mathrm{DC} \text { subgenual } \\
\mathrm{ACC} \text { for correct novel trials }\end{array}$ \\
\hline
\end{tabular}

${ }^{*}$ Effect sizes for at least one significant effect unable to be computed based on article information. ACCID, accidents; ADHD, excluded for attention deficit hyperactivity disorder; ANX, excluded for anxiety disorder; AUD, alcohol use disorder; BDI, Beck Depression Inventory; CADSS, Clinician Administered Dissociative States Scale; CAPS, Clinician Administered PTSD Scale; CBCL, Child Behavior Checklist; CD, cocaine dependence; CES, Combat Exposure Scale; CHILD, child abuse; CNV, contingent negative variation event related potential; CSA, childhood sexual abuse; CTES, Childhood Trust Events Survey; DTS, Davidson Trauma Scale; DC, depressed control group; DEP, excluded for depression; DRRI-2, Deployment Risk and Resilience Inventory; DX, diagnosis; IES-R, Impact of Events Scale Revised; ELSQ, Early Life Stress Questionnaire; ERN, error-related negativity event related potential; KSADS, Schedule for Affective Disorders and Schizophrenia, Child Version; LPP, late positive event related potential; M, excluded for medical problems; M-PTSD, Mississippi Scale for Combat-Related PTSD; MDD, major depressive disorder; MED, excluded for medication; MINI, Mini International Psychiatric Interview; N, excluded for neurological problems; N/A, not applicable; N170, N170 event related potential; N2, N200 event related potential; N4, N400 event related potential; NC, trauma-no control group; ND, natural disaster; $P$, excluded for psychiatric diagnoses; $P 1, P 100$ event related potential; P2, P200 event related potential; $P 3$, P300 event related potential; P3a, frontocentral P300; P3b, centroparietal P300; PCL, PTSD Checklist; PCL-C, PTSD Checklist, Civilian Version; PCL-M, PTSD Checklist, Military Version; PCL-S, PTSD Checklist, Specific Version; PDS, Posttraumatic Stress Diagnostic Scale; PSS, Posttraumatic Stress Scale; PSY, excluded for psychosis; PTCI, Posttraumatic Cognitions Inventory; PTSD-SS, PTSD Self Rating Scale; SCID, Structured Clinical Interview for DSM-IV; SEX, explicitly examined sex differences; SSAGA, Semi-Structured Assessment for the Genetics of Alcoholism; SUIC, excluded for suicidal ideation; SUD, excluded for substance use disorders; TBI, traumatic brain injury; TES, Traumatic Events Scale; TIME, reported time since trauma; TERROR, terrorist attack; UNKN, unknown; VAR, various traumas; VET, veterans; YC, trauma-yes control group. 

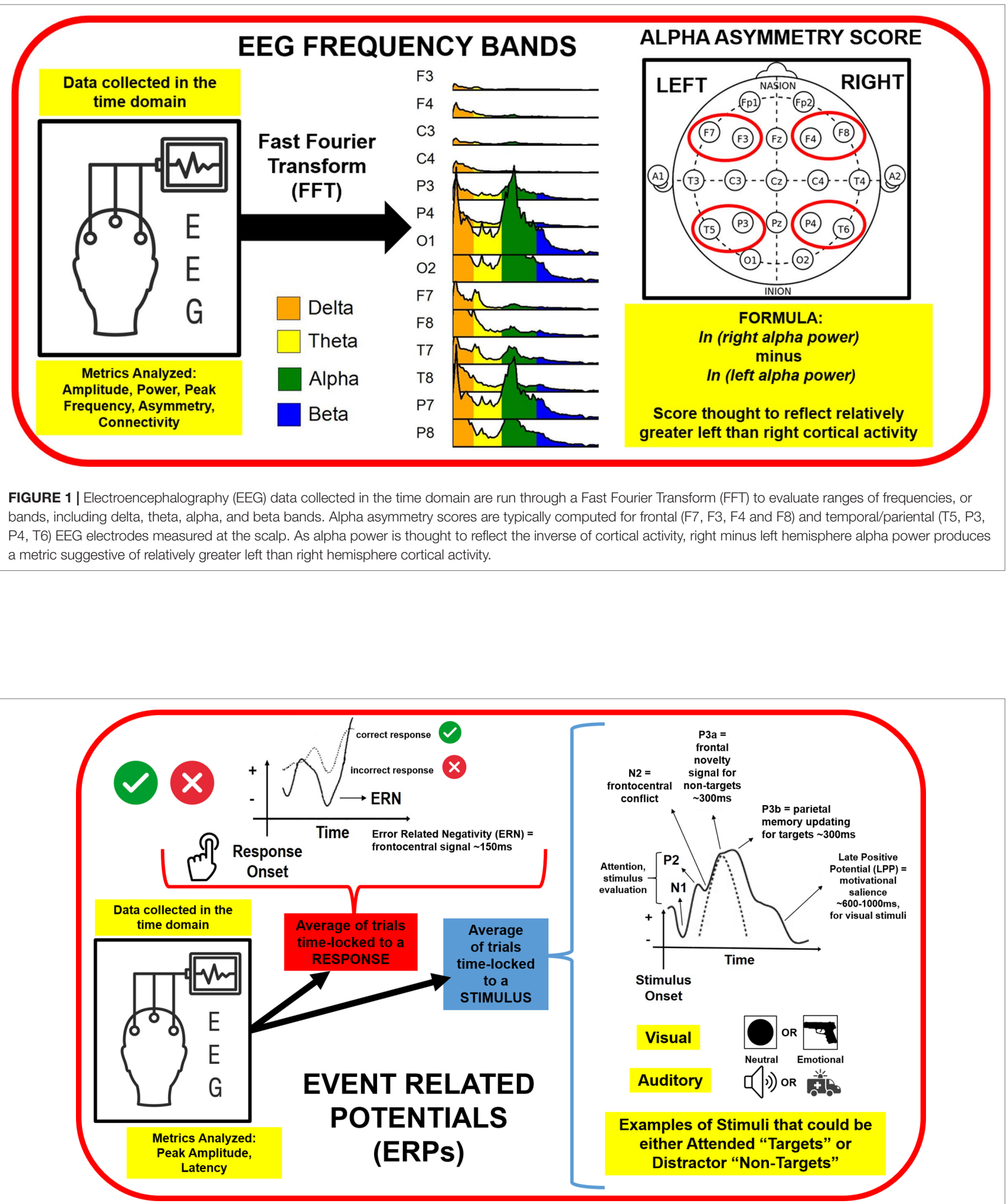

FIGURE 2 | Continuous electroencephalography (EEG) data are segmented into trials time-locked to a particular stimulus or response, and then these trials are averaged together to evaluate the peak amplitude and latency of various event related potentials (ERPs). Auditory and visual stimuli that are neutral and emotional or trauma-related have been employed to study attention, stimulus evaluation, conflict and novelty processing, memory updating, and motivational salience as a function of trauma exposure. ERPs to incorrect responses have also been explored to evaluate links between trauma experiences and error monitoring. 
delta, theta, alpha, beta, and gamma; frontal and parietal brain asymmetry is also computed by computing alpha band differences between the hemispheres. Table 2 provides a summary of effect size magnitude for significant EEG frequency results, whereas Table 3 provides detailed information for EEG studies examining trauma within the context of various frequencies, including those involving frequency bands outlined above [see also (139), for a review on resting state EEG frequency bands in psychiatric disorders]. On the whole, published reports linking EEG activity to PTSD diagnosis/symptoms are inconsistent, with the exception of alpha band-related parietal EEG asymmetry. The majority of EEG frequency studies collect brainwave recordings while individuals are in an uncontrolled "resting state" with their eyes closed or open, in the latter case focusing their eyes on a fixation cross in the center of a computer screen. Although it has been argued that these resting state recordings may reflect trait characteristics (140), research demonstrates that resting EEG consists of $\sim 40-50 \%$ state variance, likely due to heterogeneity in individual participant cognitions ("mind wandering") and arousal levels during recording (141). Inconsistent resting EEG results across studies may be due, in part, to state-related variations across individuals that dominate over clinical group or symptom differences.

\section{Delta Band}

Whereas spontaneous delta band activity is associated with rapid eye movement sleep and may reflect processes implementing basic biological functioning (142), blunted delta oscillations elicited during various active tasks are thought to index deficits in cognitive functioning $(143,144)$. Delta oscillations Although greater early life trauma is linked to lower delta power (35), additional studies report no delta power differences between PTSD+ and PTSD- with or without trauma exposure $(47,53$, 97). More research is warranted to determine whether early life trauma results in developmental disruptions to the brain that may be marked by heightened delta signals.

\section{Theta Band}

Theta band oscillations are associated with cognitive processes such as working memory load and sequencing as well as encoding of spatial and temporal information (145-147). Similar to delta band findings, resting state theta band results are also mixed, with studies showing higher theta power in PTSD+ than PTSDwith and without trauma exposure $(61,97,101)$, no differences between PTSD+ and PTSD- with or without trauma exposure $(47,53)$, or the opposite pattern, wherein greater early life trauma and PTSD+ status are both linked to lower theta power $(35,105)$. As PTSD+ were compared to PTSD- without similar trauma exposure in (105), perhaps trauma experience alone in some circumstances, but not PTSD, is linked to reductions in cognitive control processes indexed by theta power. Attenuated theta power may manifest in difficulty maintaining information in working memory or difficulty evaluating negative consequences of behavior, but more research on theta oscillations within the context of task-based paradigms may elucidate the role of theta signals in PTSD.

\section{Beta and Gamma Bands}

Beta oscillations are thought to reflect active control of an individual's current cognitive or motor state, as well as anticipation of changes to this state; it is argued that heightened beta power may reflect cognitive or behavioral inflexibility (148). Similar to delta and theta results, resting state beta band findings are incongruent, with two studies showing that early life trauma and PTSD+ status are negatively correlated with beta power (35, 98) but others reporting: 1) no differences between PTSD+ and PTSD - without trauma histories (53); or 2) opposite findings, with PTSD+ displaying greater beta power than PTSD- without similar trauma experiences (97). Faster than beta rhythms, gamma oscillations are present during various cognitive processes and are argued to reflect multisensory stimulus perception, evaluation, and representations in short- and longterm memory; similar to beta signals, gamma signals are also linked to processing of motor responses (149). One study reports that PTSD+ exhibit lower resting state beta and gamma band connectivity strength than PTSD- without trauma experiences, a neural pattern associated with greater depression, arousal, and other trauma symptoms (48). In contrast, another study reports that PTSD+ is linked to higher gamma band activity than PTSDwho had also experienced traumatic events (99). As of now, there is no cohesive theory explaining how alterations in these frequency bands relate to PTSD symptoms or trauma processing. Future evaluation of theta, beta, and gamma oscillations during specific cognitive or emotional tasks, as opposed to uncontrolled resting state recordings, may elucidate potential perceptual, motor, and memory impairments linked to PTSD.

\section{Alpha Band}

Alpha is the most commonly studied of the frequency bands, with alpha power, peak frequency, asymmetry, and connectivity being investigated. It is argued that increased alpha power reflects suppression of task-irrelevant processes, whereas decreased alpha power reflects the release from inhibition, or in other words, degree of cortical activation $(147,150)$. For overall alpha power, null group findings between PTSD+ and PTSDwith or without trauma histories $(47,53,97)$; again conflict with other studies demonstrating that early life stress and PTSD+ status are associated with lower alpha power $(35,46)$. Although PTSD+ status and early life trauma are both linked to higher alpha peak frequency $(35,47)$, it is unclear how peak frequency differences conceptually relate to trauma symptoms. In summary, the relevance of overall alpha power across the scalp to PTSD symptoms is unclear.

\section{Alpha Brain Asymmetry}

Hemispheric differences in alpha band activity are often studied using an EEG asymmetry score, which subtracts left from right alpha power; as alpha power is thought to reflect the inverse of cortical activity, positive scores on this asymmetry metric indicate increased activity in the left hemisphere, and negative scores indicate increased activity in the right hemisphere (151, 152). Frontal EEG asymmetry patterns measured during a resting state are thought to reflect individual differences in motivational 
and emotional styles, with increased leftward activity indicating heightened approach-related motivation/emotion, and increased rightward activity reflecting heightened withdrawal-related motivation/emotion $(140,153)$. As core symptoms of PTSD and traumatic reactions reflect increased negative emotionality, it would be expected that presence of PTSD and/or severe trauma symptoms would be associated with relative right frontal asymmetry. Indeed, relative right frontal asymmetry is linked to greater symptom severity of: 1) childhood maltreatment in girls $(40,41)$ and 2) PTSD+ in a mixed-sex sample (44). Moreover, a novel longitudinal study measuring EEG asymmetry and clinical symptoms three times within a 2 -year period indicates that for girls with low child trauma severity, rightward frontal EEG asymmetry across timepoints is associated with future development of PTSD (104).

However, results are far from consistent, particularly for males. Increased trauma severity in boys is associated with increased left frontal asymmetry (40); however, PTSD+ and PTSD- do not differ in males with a history of combat-related trauma (47). Mixed-gender samples also show conflicting findings, with PTSD+/PTSD- trauma-exposed individuals exhibiting greater left frontal asymmetry than PTSD- nonexposed participants (43), PTSD+ showing similar asymmetry patterns as PTSD- without traumatic histories (103), and nonsignificant relationships between frontal asymmetry and trauma, depression, and anxiety severity (100). Lack of consistent findings could be related to: 1) sex differences in frontal asymmetry previously reported in the literature, with depression linked to left frontal asymmetry in men but right frontal asymmetry in women (154), divergent patterns that may be obscured by not directly comparing males and females in statistical analyses; 2) use of a $\mathrm{Cz}$ reference montage (100), shown to be the least consistent in producing reliable asymmetry results (152); and 3) recruitment of "super controls" who differ on various personality/mood variables from PTSD+ individuals (103).

Some researchers argue that individual differences in motivation/emotion associated with EEG asymmetry may be more reliable and robust when measured during emotional challenges than resting states, as these challenges may directly engage frontal regions involved in these motivational tendencies $(155,156)$. Thus, with the context of active tasks, it would be expected that PTSD+ status and/or severity is associated with greater relative right frontal asymmetry, reflective of heightened withdrawalrelated motivation/negative emotion processing. Indeed, studies demonstrate that higher right frontal asymmetry is linked to: 1) more severe PTSD, rumination, and depression symptoms when viewing negative images (43); 2) greater emotional intensity and physical reactions to trauma images (43); and 3) PTSD+ during trauma image viewing, compared to PTSD- (42).

Parietal EEG asymmetry, in contrast, is thought to index levels of arousal as opposed to valence, wherein greater right than left parietal activity reflects higher arousal $(157,158)$. Although depression is linked to under-arousal reflected by greater left than right parietal activity, findings are inconsistent and may be influenced by state factors such as recent caffeine intake (156). Although PTSD is characterized by increased negative affect (depression), hyperarousal (associated with anxiety) is also a core component of the disorder, suggesting that relative right parietal asymmetry may more accurately index PTSD symptoms. Three studies show results consistent with this hypothesis, wherein greater right parietal asymmetry is linked to greater childhood maltreatment severity in boys and girls (40), higher comorbid depression and arousal symptoms in PTSD+ women (45), and PTSD+ individuals, as opposed to PTSD- participants diagnosed with MDD who showed lower right parietal asymmetry (44). Moreover, recent work demonstrates that PTSD+ display greater midline-to-right hemisphere parietal alpha connectivity than PTSD- (101) as well as greater alpha suppression in right parietal regions (61), findings which may help to explain the right versus left hemisphere imbalance in the parietal lobes. In addition to resting state recordings, parietal asymmetry patterns emerge during emotional challenges, wherein PTSD+ display greater right than left parietal activity than PTSD- and higher PTSD symptom severity is linked to higher relative right parietal asymmetry during trauma image viewing (42). On the whole, state emotional challenges targeting approach and withdrawal mechanisms may provide more consistent, robust biomarkers of PTSD pathophysiology than EEG recorded during a resting state.

Finally, type of trauma experience may play a role in heterogeneity of EEG results. Although PTSD+ and PTSDcombat veterans do not differ in frontal or parietal alpha asymmetry (47), other studies indicate that PTSD- who have experienced other types of traumatic events (accidents and witness to injury/death) similar to PTSD+ exhibit greater left frontal alpha asymmetry than PTSD+, a pattern thought to reflect resilience, or protection against deleterious effects of stress (42, $43,102)$. Perhaps prolonged exposure to certain types of trauma such as combat alters brain function regardless of the degree of clinical symptoms experienced, although more research is warranted to test this hypothesis.

\section{Unconventional Electroencephalography Analysis Approaches \\ Microstates}

Traditional frequency band analysis consists of averaging power within a particular band over several minutes of EEG recording. It is argued that this trait-like measurement may miss out on faster, dynamic shifts in neural activity, termed microstates, which may capture variance linked to psychopathology. During a resting state, individuals transition to and from microstates, or specific patterns of electrical activity evident across the scalp, thought to reflect oscillations within specific networks (159). Microstates are quantified by calculating global field power, the standard deviation of electrical strength across all electrodes at a particular moment in time. Researchers can then plot global field power as a function of time across a set of frequencies (e.g., alpha band) to generate topographic maps that can be submitted to cluster analysis; results from this analysis provide information on how long each individual stays in a particular microstate and how often the person transitions in and out of these microstates (159). Much more research is needed to determine the functional relevance of each microstate and how it relates to various clinical constructs. A recent innovative study in this regard indicates that 
combat PTSD+ differ from combat-exposed PTSD- on three of eleven EEG microstates emerging from feature analysis; these microstates were then correlated with simultaneously recorded functional magnetic resonance imaging (fMRI) activity and PTSD symptoms to enhance interpretation of results (160). Specifically, two findings link EEG/fMRI activity with PTSD symptoms: 1) PTSD+ spend more time in a microstate associated with the medial prefrontal cortex (a component of the brain's default mode network) than PTSD-, and within PTSD+, this greater duration is linked to higher PTSD symptom severity; and 2) PTSD+ spend less time in a microstate associated with insular and cingulate cortices (components of the brain's salience network) than PTSD-, and within PTSD+ lower duration in this state is linked to higher anhedonia, or loss of pleasure (160). These results suggest that individuals with more severe PTSD symptoms are internally hyper-focused and paying less attention to externally relevant stimuli. If these microstates predict treatment outcome or can be changed as a function of treatment, EEG could be used to track these metrics.

\section{Non-Linear Electroencephalography Dynamics}

Examining relationships in brain activity between and within electrodes over time can highlight whether electrical complexity and connectivity between cortical regions are disrupted as a function of trauma. Compared to PTSD- without trauma exposure, civilian PTSD+ show higher connectivity (correlations between electrodes) within the left hemisphere but lower connectivity within the right hemisphere; this pattern is thought to reflect attenuated neural processing complexity in the right compared to the left hemisphere (161). Another study used non-linear modeling to determine that civilian PTSD+ exhibit lower complexity than PTSD- without trauma exposure in several electrode locations, most within the right hemisphere (162). These findings point to right hemisphere dysfunction in PTSD consistent with alpha band asymmetry results. A recent study also demonstrates that PTSD+ exhibit reduced functional connectivity within delta, theta, and beta bands than PTSD- without a trauma history; EEG source analysis localized one connectivity metric to a right centroparietal region, with lower connectivity linked to higher IES-R PTSD symptoms (163). These results point to the importance of investigating information processing impairments in brain networks as well as specific brain regions in the study of psychopathology more generally and PTSD symptoms in particular.

\section{ELECTROENCEPHALOGRAPHY IN THE TIME DOMAIN: AN OVERVIEW OF EVENT RELATED POTENTIALS}

ERPs, illustrated in Figure 2, are segments of EEG that are averaged over repeated trials to characterize the neural response to a particular event, such as a stimulus, response, or absence of a response (e.g., inhibiting a button press). ERPs are typically analyzed by investigating differences in amplitude and latency between stimulus conditions and/or groups of subjects. ERP amplitude is often defined as the difference between a pre-stimulus baseline and the largest waveform peak within a particular time-frame, whereas ERP latency comprises the time from stimulus onset to peak amplitude $(164,165)$. The components we will review in relation to PTSD include N170, P200, N200, P300, N400, contingent negative variation $(\mathrm{CNV})$, mismatch negativity (MMN), error-related negativity (ERN), and late positive potential (LPP). These particular ERP components are described within the context of relevant paradigms below. EEG recording montages vary to the degree that electrical signals at the scalp manifesting in ERPs are able to be localized to their sources within the brain. EEG/ERP source localization studies typically employ 64 or more scalp electrodes of spatial resolution to more accurately estimate the origins of electrical signals, often in conjunction with magnetic resonance imaging or magnetoencephalography methods. As EEG/ERP source localization studies specifically within PTSD samples are relatively uncommon, for various ERP components we cite their potential sources based on other healthy or clinical samples.

\section{EVENT RELATED POTENTIAL MARKERS OF TRAUMA EXPERIENCE AND POSTTRAUMATIC STRESS DISORDER SYMPTOMS}

Common paradigms employed to evaluate ERPs as a function of trauma and PTSD status include auditory and visual oddball, Stroop, flanker, go/nogo, facial identification, and passive listening/viewing tasks. Historically, oddball tasks have focused on basic tones or visual shapes not associated with trauma or emotional significance; these tasks help to address whether a particular processing deficit generalizes across all stimuli, or is just specific to trauma. While Table 2 provides an overview of effect sizes for auditory and visual ERPs in the same chart, going forward we review ERP component findings for auditory (Table 4) and visual (Table 5) modalities separately. Below we cluster findings as a function of stimulus modality and the specific function(s) a particular ERP component is thought to capture (attention, working memory, inhibition, emotion regulation, or reward processing). Paradigms employed by ERP researchers vary in complexity depending on the question of interest. This could include use of passive viewing paradigms versus those involving action selection and/or inhibition of these actions (i.e., Oddball tasks). Because of the differences in neural resources required for the latter process, we include inhibition as a separate section from attention and working memory paradigms. Within each type of EEG paradigm, researchers often use either neutral or trauma-relevant stimuli to ascertain the specificity versus generalized quality of findings. Results using neutral or traumarelevant stimuli are discussed within the sections describing each type of paradigm (i.e., attention and working memory, inhibition, emotion regulation).

\section{Auditory Attention and Working Memory}

During passive auditory tasks, participants listen to tones of various intensities and are not required to mentally count stimuli or make a behavioral response; within these tasks, the brain's selective attention to stimulus characteristics is the primary focus. 
P200, or P2, is a positive component peaking from 150 to $275 \mathrm{~ms}$, thought to be associated with selective attention, detection, and retrieval in short-term memory evident throughout frontocentral electrodes $(166,167)$. N200, or N2, occurs closely following P2 and is a negative component occurring around $200 \mathrm{~ms}$ in prefrontal, anterior cingulate, and superior temporal cortices (168). N2 detects deviant stimuli while attention is fixed upon a standard stimulus $(164,169)$. One study indicates that PTSD+ exhibit lower P2 amplitude to high intensity (deviant) tones than PTSDwithout a trauma history, a pattern linked to lower depression and PTSD symptom severity (51). In contrast, a second study with a larger sample demonstrates the opposite effect, wherein PTSD+ display greater $\mathrm{P} 2$ and $\mathrm{N} 2$ amplitudes to higher intensity tones than PTSD - who do have a trauma history, a pattern associated with heightened re-experiencing symptoms within PTSD+ (113). These two studies differ in type of trauma (combat versus child physical and sexual abuse) as well as inclusion criteria for their comparison groups, factors that may contribute to divergent findings.

Two-stimulus auditory oddball tasks require participants to press a button or mentally count infrequently occurring target tones in-between more frequent standard tones (thereby paying more attention to target than standard stimuli). The MMN ERP component captures the difference in neural processing between these standard and target stimuli. MMN is usually largest in amplitude over the central and frontal electrodes at 120 ms, suggesting that it is the mismatch detector, a pre-attentive processing of deviance in short-term memory. MMN is also thought to be reflective of a sensory memory system $(62,109$, 114). With respect to early sensory processing of deviant stimuli, PTSD+ exhibit greater MMN amplitude to targets than standards when compared to PTSD- with or without trauma histories (61, 62, 109, 114); furthermore, exaggerated MMN amplitude is linked to greater PTSD symptom severity (62). In contrast to MMN results, N2 amplitude results also indexing deviance are mixed, with PTSD+ exhibiting N2 target amplitudes higher (50, 62) and lower (53) than PTSD- without trauma histories, paired with longer N2 latency $(50,108)$. As greater trauma severity is linked to lower N2 target amplitude (35), it would be worth investigating N2 amplitude further in studies explicitly matching PTSD+ and PTSD- on trauma history.

In addition to MMN, P2, and N2 ERP components, P300, or P3, is a positive waveform peaking around $300 \mathrm{~ms}$, is elicited to in response to an attended target stimulus, prior to response generation. P3 is thought to reflect memory updating, information delivery, stimulus evaluation, active discrimination, and motivational salience on the basis of stimulus context (164, 165, 169). P3 is divided into two subdivisions: P3a and P3b. $\mathrm{P} 3 \mathrm{a}$ is typically largest in frontal and central areas to non-target stimuli in more complex oddball paradigms (it is not included in the two-stimulus oddball), whereas P3b is activated in the temporal, parietal, and cingulate cortices and is typically largest to oddball targets when compared to standards as well as other stimuli $(168,170)$. The most robust outcome of two-stimulus oddball studies is that PTSD+ exhibit lower target P3b amplitude than PTSD- with trauma histories $(54,55)$ and PTSD- without trauma histories $(50,53,56,58,108)$ with lower P3b amplitude linked to heightened avoidance/numbing, re-experiencing, and hyperarousal symptoms $(50,54,58)$. Of note, acute stress disorder (ASD), thought to be a precursor to PTSD, is linked to greater target tone P3b amplitude than PTSD+ and PTSD-, and although the latter groups do not differ, direction of group means suggests that PTSD+ displays the lowest P3b amplitude out of the three groups (107). These results point to the fact that trauma recency may be an important moderator of the brain's auditory attention response. In contrast, all but one study (50) found no differences in P3b target latency as a function of PTSD diagnosis $(51,54,56,58)$. In summary, most findings point to reduced neural resources devoted to auditory working memory updating, reductions that are worsened as a function of symptom severity, specifically for intrusions, dissociation, and hypervigilance.

In three-stimulus oddball tasks, in addition to infrequent target and frequent standard stimuli, an infrequent non-target is added that, unlike the target, does not require a behavioral response. Addition of this non-target allows researchers to study degree of neural resources devoted to relevant (target) versus irrelevant (non-target) attentional capture while simultaneously controlling for frequency of stimulus presentation. Four-stimulus oddball tasks include two non-targets instead of one, often comparing stimuli with differing emotional qualities (positive versus negative emotion). On the whole, the addition of non-target stimuli results in less consistent results as a function of PTSD status, although these studies are predominantly characterized by combat trauma samples being compared to PTSD- with trauma histories. Although some work indicates that P3b target and P3a non-target amplitudes do not differ between PTSD+ and PTSDtwins with and without trauma histories (110) or PTSD- with trauma experience $(111,115)$, additional studies report that PTSD+ exhibit lower or higher (49) P3b target amplitude than PTSD- who have experienced trauma. However, a small sample of PTSD+ shows auditory P3a reductions as a function of eye movement desensitization and reprocessing (EMDR) therapy, whereas a PTSD- administered sham therapy showed similar P3a amplitudes pre- and post-assessments (112). Perhaps type of trauma plays a role in inconsistent findings, as Metzger et al. (57) include childhood sexual abuse as well as combat trauma, whereas Metzger et al. (49) focus solely on combat-related PTSD.

It is important to note that non-target results discussed thus far consist of deviant tones unrelated to traumatic events; perhaps trauma-relevant non-target stimuli evoke a more powerful neural response than non-relevant tones. To this end, higher P3a trauma-related non-target amplitudes are associated with greater hyperarousal symptoms in individuals located near (59) but not far from a natural disaster (60). Furthermore, greater PTSD avoidance and hyperarousal symptoms related to a traumatic fire are linked to greater P2 and P3a amplitudes to both positively and negatively valenced non-target stimuli (106), suggesting that exaggerated attention is paid to emotionally arousing stimuli in PTSD, particularly for individuals experiencing hypervigilance to threat.

\section{Auditory Inhibition}

Within the context of an inhibition paradigm, two mismatched tones (of different frequencies) signal that the participant should make a behavioral response (go), whereas two matched tones 
(of the same frequency) signal the participant to withhold a behavioral response (nogo). High levels of comorbid depression and trauma in a non-clinical sample are associated with longer frontal but shorter centroparietal P3 latencies to go stimuli, but it is unclear whether these findings are driven by trauma, depression, or both (63). A more complex auditory go/nogo variant consists of a continuous performance task wherein participants hear a stream of letters and must press a button (go) when they hear an "X" when it occurs after an "A," inhibit a button press when they hear any other letter following an "A" (nogo), and ignore taskirrelevant letters (non-targets). Despite no group differences in P2 or P3 amplitude to go or nogo stimuli, PTSD+ exhibit longer P3 latency to nogo stimuli than PTSD- without trauma history, a pattern that is linked to greater hyperarousal symptoms. Moreover, PTSD+ show greater frontal P3a amplitude to irrelevant nontargets than PTSD- (91). These findings, while limited, suggest that trauma is associated with delayed stimulus discrimination and greater resource allocation to novel distractors.

\section{Visual Attention and Working Memory}

Substantially more ERP trauma research has focused on attention and working memory processes within the visual modality than the auditory modality, with the majority of these studies measuring brain responses to emotionally valenced images or faces. Visual stimuli elicit P2, N2, and P3 amplitudes similarly to auditory stimuli, although the latency of component onset may be slightly delayed compared to auditory ERPs. In addition to the aforementioned components elicited by auditory stimuli, the LPP, a late positive ERP component visible from 400 to $1,000 \mathrm{~ms}$, is modulated largely by the emotional content of visual stimuli; positive or negative images elicit larger LPP amplitudes (greater positivity) than neutral images (171). Simultaneous EEG and fMRI recordings demonstrate that LPP amplitude is positively correlated with signal increases in visual cortex, amygdala, and prefrontal cortex (172). With respect to these ERP components, studies involving passive image viewing provide mixed results. Although greater PTSD avoidance symptoms are associated with lower P3 and LPP amplitudes for traumatic than neutral images (75), PTSD+ show no differences from PTSD- in LPP amplitude to positive, trauma, or neutral images, despite exhibiting attenuated P2 amplitudes to these stimuli (67). A novel study was able to study the same individuals before and after their experience with a natural disaster, demonstrating that although PTSD- exhibit smaller LPP amplitude to negatively valenced images after as opposed to before the trauma, PTSD+ display no change in LPP amplitudes pre- to post-trauma, signaling exaggerated responses to aversive events (119). It may be the case that PTSD characterized by exaggerated avoidance of thoughts and feelings reminiscent of trauma reduces neural resource allocation to threatening stimuli, at least in the short term.

Research suggests that face processing may be altered as a function of exposure to trauma, although findings are inconsistent as to whether alterations are present across faces or specific to emotional expressions signaling threat. N170 is a visually evoked negative component occurring at $170 \mathrm{~ms}$ that is enhanced to face stimuli (173). Conflicting N170 results exist as a function of traumatic experiences. Although childhood trauma is linked to smaller N170 amplitude to angry/fearful as opposed to happy faces $(83,85)$, both combat-related trauma and PTSD+ are associated with heightened N170 amplitudes to faces more generally (84) but no N170 amplitude change to angry faces as a function of pre- versus post-deployment in PTSD+ and PTSDwith similar combat trauma (65). The P2 literature is equally messy in that PTSD+ display either attenuated P2 amplitude to happy faces $(66,121)$ but enhanced P2 amplitude to various faces when compared to PTSD- with traumatic brain injury (TBI) (123). Finally, PTSD+ display greater P2 amplitude to angry faces than PTSD- post- as opposed to pre-deployment (65). With respect to P3 differences, PTSD+ display longer frontocentral P3 latency to happy faces than PTSD- with history of trauma (99) as well as larger P3 amplitude to neutral than angry faces during a dot probe task (71). During later elaborative processing, PTSD symptoms in combat veterans are linked to smaller LPP amplitude to angry faces $(66,78,79)$. Inconsistent results may be due, in part, to varying task requirements, type of trauma studied, and exclusion criteria related to medication status, SUD, and depressive symptoms.

Additional visual discrimination paradigms pair emotional, neutral, and/or trauma images with an active task requirement that involves making a choice based on other irrelevant features of the images (e.g., whether an image includes food, contains a certain color, or is located in a specific spatial location). Trauma experience more generally is associated with larger P2, P3, and LPP amplitudes to trauma as opposed to neutral images $(64,127)$ as well as larger LPP amplitude to negative than neutral images (80, 174); the latter pattern also predicts higher future externalizing symptoms in those reporting the most severe trauma symptoms (80). Clinical studies, on the other hand, provide inconsistent results, with $\mathrm{PTSD}+$ showing no difference in $\mathrm{P} 3$ amplitude to emotional or neutral images than PTSD- without a history of trauma, but displaying heightened LPP to positive images (77) or no group differences in LPP amplitude to threat (117). In contrast, PTSD+ display greater P3 and LPP amplitudes to food, neutral, and trauma images than PTSD- who share a history of combat-related trauma (122); as both studies possessed small sample sizes, it is unclear how to reconcile these results.

An ERP component employed to study anticipation of an upcoming stimulus is the CNV, a slow negative wave associated with working memory and motor/perceptual timing (175). While typically recorded in frontocentral regions, multiple brain areas are thought to contribute to CNV formation including prefrontal, primary motor, and primary somatosensory areas (176). During a choice reaction time task, PTSD+ show greater CNV amplitude to negative stimuli than PTSD- without a history of trauma, with higher PTSD severity and re-experiencing symptoms linked to higher CNV amplitude (82). These findings suggest that exaggerated neural resources are being allocated to past and future aversive events as a function of PTSD, although additional research is warranted to determine if results generalize to trauma experience or solely a function of PTSD.

Two, three, and four visual oddball conditions are analogous to auditory oddball designs, with most visual studies focusing on P3 differences as a function of trauma or PTSD diagnosis. Two-stimulus 
oddball studies report that lower target $\mathrm{P} 3 \mathrm{~b}$ amplitude differentiates PTSD+ from PTSD- (70) and also predicts future conversion from PTSD- to PTSD+ (74). In contrast, for individuals who have experienced combat trauma, P3b amplitude increases over time parallel reductions in PTSD and depression symptoms within the same window, whereas no changes in P3b amplitude are associated with increased symptoms (76). Three- and four-stimulus oddball paradigms demonstrate that PTSD+ exhibit larger P3a amplitudes to traumatic non-targets than PTSD- both with and without trauma histories $(68,72,124)$. Moreover, both PTSD+ and trauma history appear to be associated with smaller P3b target amplitude $(68,73)$. Findings for P3 latency are mixed, with studies reporting slower (124) or faster P3a latency to trauma (72), paired with slower (116) or faster (68) P3b latency to targets.

In addition to oddball tasks, researchers have used various word-based paradigms to assess short-term memory function linked to trauma experience. When performing a Sternberg item-recognition task involving the maintenance of a word list in short-term memory and then differentiating these words from new words, PTSD+ with comorbid TBI and PTSD- with trauma do not differ in P3 amplitude to old versus new words when they perform this task in isolation; however, completing this task in the presence of additional cognitive load, P3 amplitude fails to differentiate old from new words in PTSD+ with TBI but not PTSD- with trauma (118). It is difficult to attribute study results specifically to a PTSD diagnosis, given that TBI presence differed between groups. During a 1-back working memory task involving letters, unmedicated PTSD+ exhibited larger P3 target amplitude than medicated PTSD+ despite no differences from PTSD- (69). During a declarative memory paradigm, PTSD+ display larger P3 and LPP amplitudes to trauma-related questions than PTSDwithout trauma, but PTSD- with trauma did not differ from either group; in contrast, PTSD+ and PTSD- with trauma show smaller frontal CNV amplitude to trauma questions than PTSDwithout trauma (125). On the whole, results do not suggest a basic visual working memory deficit specific to PTSD.

Within individuals who experienced a natural disaster, P2 and P3 amplitudes are larger for trauma-related than trauma-unrelated words, signals localizing to parahippocampal gyrus (126). N400, or N4, is a negative ERP deflection that is largest in response to unexpected information (often related to language processing) that is localized to middle, superior, and inferior temporal regions as well as dorsolateral prefrontal cortex and thought to relate to semantic memory retrieval (177). PTSD+ comprised of various traumas display smaller N4 amplitude to threatening sentence endings during a sentence completion task than PTSD-, but no differences for expected or unexpected sentence endings (120), suggesting that trauma-related information is more readily accessible in semantic memory, and is therefore less unexpected. Furthermore, a recent study demonstrates that within trauma survivors, larger N400 amplitudes to negative sentence endings is linked to greater negative cognitions about the world as a result of trauma (86).

\section{Visual Inhibition}

In the color-word Stroop paradigm, individuals are instructed to read color words (congruent trials) or name the color in which these words are written (incongruent trials), and the Stroop effect involves subtracting reaction time of congruent from incongruent trials to obtain a measure of response interference (178). For example, if "red" is written in green ink, congruent trials require the participant to select "red" as the correct response, whereas for incongruent trials, the correct answer would be "green." In a modified emotion-word Stroop paradigm, color-related words are replaced by positive, negative, neutral, and/or trauma-related words, and individuals are required to select the correct color in which each word is written; longer responses to emotional than neutral words are thought to reflect greater attention paid to emotional words. Color-word Stroop results indicate that greater trauma symptoms are associated with lower right prefrontal negativity from 400 to $600 \mathrm{~ms}$ for incongruent as opposed to congruent stimuli (133); these results point to impaired inhibitory processing. Three emotion-word Stroop studies also indicate that PTSD+ display smaller P3 amplitude than PTSD- with and without trauma histories across trauma, emotional, and neutral conditions $(57,87,128)$, although an additional study finds that individuals with a trauma history exhibit greater $\mathrm{P} 3$ amplitude to positive than negative words when compared to subjects without trauma, findings localized to parahippocampal gyrus and cuneus brain regions (88). On the whole, findings suggest that PTSD is characterized by reduced resources devoted to suppression of conflicting information within the context of this paradigm.

Visual go/nogo paradigms are similar to those within the auditory modality, wherein one or more cues are linked to an active behavioral response (go), and another cue or cues are linked to withholding of a response (nogo). Some variations of this paradigm also include distractor stimuli to examine whether participants are diverting neural resources to task-irrelevant information. The stop signal task also requires behavioral inhibition on some trials, but the design is slightly different: participants press a right button to one visual cue and a left button to another visual cue (go), and on a certain percentage of trials, they hear an auditory tone (signal) requiring them to withhold their response (stop). These paradigms tend to elicit larger frontal N2 amplitude to nogo/stop trials than go trials (179), which within this context is thought to reflect a heightened response to conflict (177). In addition, larger frontocentral P3 amplitude to nogo/stop trials than go trials is thought to more directly reflect inhibition of an overt motor response (181). Although some research indicates that PTSD+ do not differ in N2 or P3 amplitude to go or nogo stimuli from PTSD- with trauma histories $(89,91)$, within the context of a combat sample with comorbid traumatic brain injury (TBI), PTSD+ display larger N2 amplitude, localized to anterior cingulate cortex, than PTSD-, a pattern that is also positively correlated with PTSD symptom severity (134). However, heightened frontal P3 amplitude to nogo trials is linked to greater PTSD symptoms within a sample of police officers (90), and PTSD+ with combat trauma display greater frontal P3 amplitude to distractors than PTSD- without trauma (91). Moreover, studies indicate that delayed P3 nogo latency is associated with greater PTSD hyperarousal and avoidance (91, 92), whereas delayed distractor latency is linked to greater PTSD re-experiencing (91). One additional ERP component that is often computed for inhibition-related tasks is the ERN, a negative deflection 
occurring $150 \mathrm{~ms}$ after commission of an error, which is localized to prefrontal and anterior cingulate cortices (182). Recent work demonstrates that children with higher stress paired with larger ERN amplitude during a go/nogo task show greater internalizing symptoms following a natural disaster (94), although it is unclear whether this finding relates to trauma more generally as opposed to PTSD in particular.

The Eriksen flanker task involves suppressing distractor stimuli that are either the same (congruent) or different (incongruent) from a central target stimulus; typically participants press a button indicating the direction of the target (left or right) and this task produces more errors for incongruent than congruent trials, due to the added difficulty of inhibiting conflicting information to achieve accurate task performance. Three studies indicate that combat-related PTSD+ do not differ in ERN amplitude during flanker performance than PTSD- either with or without trauma history $(129,132,135)$. In contrast, within a non-clinical sample of individuals who experienced various types of trauma, larger ERN amplitude was positively correlated with PTSD hyperarousal symptoms, but not overall symptom severity (131); taken with ERN findings for the visual go/nogo task, perhaps heightened ERN reflects exposure to trauma more generally, although this hypothesis warrants further testing. Consistent with this idea, a recent investigation reports that greater combat exposure is associated with larger ERN amplitude after accounting for variance linked to anxiety and PTSD symptoms (93). Recent work suggests that the ERN amplitude itself may not be larger as a function of degree of childhood trauma, but instead the response to correct trials, the correct related negativity (CRN) amplitude, may parallel a greater degree of stressful life events (130). As CRN is sometimes employed in the difference score to calculate the size of the ERN, analysis of individual CRN and ERN waves may clarify patterns of results. Within a flanker task incorporating emotional distractors, PTSD is associated with heightened threat-related frontocentral positivity evident several hundred milliseconds post-stimulus (95).

Inhibition deficits as a function of a PTSD diagnosis appear to be somewhat paradigm- and ERP-component specific, with the largest effects for: a) a modified version of the Stroop task involving emotional, trauma, and neutral words; and b) P3 amplitude to words or distracting stimuli. Findings from other inhibition-relevant tasks do not point to a deficit in neural resources dedicated to monitoring for errors or withholding a behavioral response.

\section{Emotion Regulation and Reward Processing}

As few ERP studies have examined the impact of trauma or PTSD on emotion regulation and reward processing, studies utilizing these types of paradigms are reviewed together. During a directive emotion regulation paradigm, individuals with combat trauma view negative pictures within two contexts: 1 ) where they maintain negative feelings elicited by the pictures; and 2) where they use cognitive reappraisal strategies to lessen the emotional impact of the images. Although PTSD+ and PTSD- do not differ in LPP amplitude to negative pictures before or during the reappraisal condition, PTSD+ display lower LPP increases than
PTSD - for the negative-maintain condition (96). However, within a larger group of trauma-exposed combat veterans, less change in LPP amplitude during reappraisal over time parallels greater increases in PTSD avoidance, re-experiencing, and hyperarousal symptoms (136). These findings suggest that resources dedicated to adaptive emotion regulation are not impaired as a function of PTSD diagnosis, but that less LPP change over time results in increased PTSD symptoms as a function of traumatic exposure. During a delay discounting paradigm, trauma survivors display larger P3 amplitude to rewards and losses than individuals without trauma histories (137), but it is unclear as of yet whether this pattern is specific to PTSD. Finally, during reinforcement learning, ERP source localization analyses indicate that individuals with comorbid trauma and past MDD display greater anterior cingulate cortex signals to novel stimuli than individuals who meet criteria for past MDD+ but do not have a trauma history (138); again, it is unclear whether this finding globally relates to trauma history as opposed to PTSD.

\section{SYNOPSIS AND CLINICAL IMPLICATIONS}

From a categorical approach to psychopathology, the majority of EEG/ERP studies do not produce clear-cut diagnostic markers of PTSD. On the whole, the electrophysiology literature primarily reflects inconsistent findings when comparing PTSD+ and PTSDindividuals; this lack of consensus is not that surprising, given that 70,000+ symptom presentations that can result in a DSM-IV PTSD diagnosis, and over 600,000+ symptom configurations can manifest in a DSM-5 diagnosis (183). Samples across studies likely differ on the severity of depression, avoidance, hyperarousal, re-experiencing, and other comorbid symptoms that could impact results. With respect to additional clinical issues, Tables 3-5 illustrate that the majority of studies investigating trauma exclude individuals with medical and neurological problems (including brain damage) and at least some degree of psychiatric comorbidity (often psychotic and substance use disorders) from participation, reducing some influence of potential confounds; however, these exclusions can also limit the generalizability of findings, particularly given that PTSD is often comorbid with other mood, anxiety, and substance use disorders.

Discordant findings may also be related to small sample sizes, medication effects, time since trauma (e.g., early life stress being recalled in adulthood versus assault reported last year), type of trauma experienced (e.g., accidents versus assault or combat), and potential sex differences. Approximately one-third of the studies we reviewed explicitly address medication effects in their inclusion/exclusion criteria. Furthermore, only 23\% report trauma recency; of those that do, substantial heterogeneity exists for the time since trauma exposure, ranging from 6 months to 10 years. In addition, only $10 \%$ of studies include biological sex in statistical analyses, despite the fact that EEG studies find more robust relationships between psychopathology and brain activity in women than men [e.g., Refs. $(154,184)]$. Only $45 \%$ of studies compared PTSD+ to PTSD- who had actually experienced a similar traumatic event, thereby better isolating the impact of exaggerated responses to the trauma (clinical symptoms) as 
opposed to the trauma experience itself. Given considerable heterogeneity across individuals diagnosed with PTSD, it may be more realistic to focus on patterns of brain activity that are linked to specific symptoms of distress/impairment to be targeted in biological and psychological interventions. With large enough samples, medication presence/absence, sex, and time since trauma can all be included within models investigating relationships between brain activity and symptom dimensions.

Furthermore, although we attempted to illustrate the magnitude of various EEG and ERP relationships to trauma and PTSD in Table 2, results from Tables 3-5 demonstrate that many of these studies do not report exact $p$-values and/or effect sizes, limiting quantitative conclusions regarding which electrical patterns are the most robust predictors of PTSD symptoms and/ or outcomes. The majority of the effect sizes included in Tables 3-5 were calculated by the senior author based on means/ standard deviations or correlation coefficients included in each published article; in other words, these studies are not explicitly reporting effect sizes in their articles. To propel the field forward and encourage replication and ease of comparison, it is crucial that studies report effect sizes so that researchers and clinicians can gauge magnitude of various findings.

When we take categorical and dimensional EEG/ERP approaches together, four main patterns emerge. First, rightward frontal asymmetry has been identified as relating to PTSD diagnosis and symptoms and more specifically, social isolation/withdrawal and heightened negative affect. Given that these dimensions span not only PTSD but also other disorders such as MDD and PD suggests this may serve as a transdiagnostic marker of symptom severity. Future research can evaluate whether PTSD treatment interventions reduce this asymmetry as well as withdrawal-related symptoms of depression, rumination, and self-blame.

Second, PTSD diagnosis, symptoms, and trauma severity are linked to rightward parietal brain asymmetry. Research indicates that higher alpha power in right parietal cortex reflects greater internal attentional focus and orienting away from external distractors (185). As PTSD symptoms are associated with relatively lower alpha power (greater cortical activity) in right than left parietal regions, this imbalance may be linked to difficulty orienting attention away from distracting stimuli in the environment that are irrelevant to goal-directed behaviors. As individuals with PTSD have difficulty differentiating between safe and threatening cues, overestimate the probability of threatening events, and generalize threat responses across similar types of stimuli (186), it is possible that right parietal dysfunction contributes to or reflects this generalized hypervigilance and threat overattribution. A recent fMRI study demonstrates that within a sample of combat veterans, greater pre-treatment left inferior parietal activation during a stop signal task is associated with greater PTSD symptom reductions as a function of psychotherapy (187). Taken together, studies point to hemispheric patterns of parietal asymmetry as promising diagnostic and predictive markers of PTSD symptoms and treatment outcome, although more research is needed to replicate and extend these findings.

Third, individuals with PTSD show attenuated allocation of neural resources (often within parietal cortex) to process auditory/ visual target (i.e., goal-relevant) and trauma stimuli within the context of memory updating (P3b amplitude) and motivational attribution (LPP amplitude; see Table 2 for $20+$ effects using at least one of these metrics); this effect is especially pronounced for those endorsing high avoidance symptoms. Perhaps exposure therapy could be enhanced by augmentations that bolster neural circuitry to enhance working memory and motivational focus (188).

Fourth, exaggerated processing of novel, emotional, trauma, and/or task-irrelevant auditory and visual stimuli specifically within frontocentral brain regions (CNV, MMN, and P3a amplitudes) is linked to greater PTSD re-experiencing, hypervigilance to threat, and overall symptom severity. These findings point to PTSD as a disorder of attention dysregulation-difficulty differentiating between task relevant and irrelevant information-that could contribute to problems concentrating and focusing on daily tasks (family/social interactions, school, or work) instead of distractors that could be interpreted as threatening and trauma-relevant (189). These distractors may trigger further intrusions and negative emotions that individuals find difficult to control.

\section{REDUCING IMPACT OF THE ELECTRICAL AFTERMATH}

Frontoparietal circuitry important for attentional processing across various tasks appears to be disrupted as a function of PTSD symptoms. Do changes in this circuitry parallel symptom reductions and can ERPs predict treatment response? Recent work demonstrates that the answer is yes on both counts. For instance, MMN and P3a amplitudes are malleable as a function of auditory-based targeted cognitive training in individuals with schizophrenia, with ERP changes predicting verbal learning improvements and reductions in clinical symptoms $(190,191)$. Moreover, $\mathrm{P} 3 \mathrm{~b}$ amplitudes predict treatment outcome and relapse for individuals with SUDs (192). Within trauma samples, fMRI research indicates that psychological and biological interventions appear to modulate activity and processing in frontal and/or parietal regions, resulting in promising PTSD symptom reduction [e.g., Ref. (2)]. Limited EEG research on prediction of treatment outcome suggests that: 1) CBT may result in a reduction of right frontal activation to trauma-relevant stimuli [an effect correlating with symptom reduction (193)]; 2) eye movement desensitization and reprocessing (EMDR) may elicit a reduction of the $\mathrm{P} 3 \mathrm{a}$ component, suggesting a normalization during orienting to novel stimuli (112); and 3) transcendental meditation may increase EEG power within low-frequency bands (194). However, these studies suffered from small samples (less than 20 per group) and/or lack of comparison groups; thus, further work is needed in larger studies to assess the robustness of these findings. In the sections below we explain potential novel treatments that may target the EEG components implicated in the PTSD literature and highlight how EEG/ERP methodology can play a role in alleviating aversive symptoms linked to trauma exposure.

\section{Brain Stimulation}

Noninvasive brain stimulation techniques such as repetitive transcranial magnetic stimulation (rTMS) and transcranial direct 
current stimulation (tDCS) show promise for altering attentional deficits linked to prefrontal cortex dysfunction. rTMS delivers a fluctuating magnetic pulse typically within the range of delta, theta, and alpha frequency ranges $(1-10 \mathrm{~Hz})$ to produce changes in neural activity; particular cortical regions are often targeted via the scalp with the use of an EEG cap. Although traditional rTMS requires 20-30 min to produce effects, theta burst stimulation (TBS) is a type of rTMS that applies continuous $5 \mathrm{~Hz}$ pulses to the brain, produces neural changes under $5 \mathrm{~min}$, and is shown to influence performance on executive function tasks, particularly those involving working memory (195). Although no published studies have reported TBS results within samples of PTSD patients, a recent meta-analysis (196) indicates that frontal TBS effectively decreases depression symptoms that are often elevated in individuals with PTSD. Whereas rTMS/TBS applies magnetic pulses to the cortex, tDCS applies low frequency electrical currents via electrodes to a particular brain area to induce intracerebral current flow (197). Within combat veterans with PTSD, brain stimulation of prefrontal cortex alone or paired with cognitive therapy is associated with P3a normalization (reductions) to trauma-related stimuli [tDCS: Ref. (198); rTMS: Ref. (199)] as well as greater PTSD symptom reductions than cognitive therapy plus sham rTMS (200); available work suggests that targeted stimulation of right prefrontal regions may provide a more robust effect than left or bilateral stimulation $(199,200)$.

\section{Real-Time Electroencephalography Neurofeedback}

Another promising technique for reducing PTSD symptoms is realtime EEG neurofeedback, wherein patients are given instructions to increase or decrease activity within a certain frequency band at a particular cortical region; EEG data are processed immediately during recording and patients are notified of their degree of success in changing their activity. In some contexts, patients are provided with examples of cognitive/emotional strategies that may assist in changing their brain activity, such as thinking of positive memories or future plans, or engaging in a working memory task. There have been recent reviews summarizing findings from clinical outcome studies using EEG neurofeedback for PTSD $(201,202)$. These reviews highlight the limitations of the field, including small sample sizes, lack of placebo controls, and/ or lack of randomization to intervention conditions. The protocols often include training related to alpha/theta wave ratio (aiming to upregulate theta and downregulate alpha), alpha waves only, or thalamic inhibitory mechanisms. Initial evidence suggests that EEG biofeedback may result in PTSD symptom improvement, but findings are far from consistent across studies.

The use of fMRI concurrently with EEG may enhance the spatial targeting of neurofeedback. One research team recorded fMRI before and after 30 min of EEG neurofeedback focused on suppression of parietal alpha band activity in individuals with PTSD; within this study, patients were not informed of particular strategies to use or which frequency band to change, but were just presented with visual feedback of a spaceship that moved when participants were in the desired zone of brain activity (203, 204). This EEG neurofeedback targeting alpha band activity was associated with lowered hyperarousal symptoms, as well as enhanced connectivity between the prefrontal cortex and the amygdala, a subcortical brain region implicated in fear processing and aversive memories that is often overactive in PTSD $(203,204)$. As higher coactivation of prefrontal cortex and amygdala is linked to lower negative affect when healthy individuals are asked to regulate their emotions to negative stimuli (205), enhancement of this connectivity in PTSD patients may also assist in alleviating their symptoms of negative affect. As frontoparietal attentional mechanisms, particularly within the alpha band, are disrupted in PTSD patients and linked to hyperarousal, negative affect, and avoidance, available research suggests that EEG alpha neurofeedback may provide a "reset" for these mechanisms and reduce these aversive symptoms. For instance, neurofeedback using EEG/fMRI is associated with reduced PTSD symptom severity and greater leftward and bilateral prefrontal functional connectivity $(203,206)$. With respect to parietal regions, right temporal-parietal EEG neurofeedback is linked to lower tension, emotion dysregulation, and affect instability in PTSD patients than a wait-list control condition (207).

In sum, although neurofeedback is associated with PTSD symptom reduction across multiple studies, additional randomized control trials are warranted to determine how well neurofeedback performs in comparison to other treatments (201). While combined EEG and fMRI recordings allow for more precise spatial localization of brain changes than EEG alone, fMRI methodology is much more expensive and less mobile than current EEG systems, many of which are portable and can be easily set up for use in a clinic, university, or hospital. As EEG-fMRI evidence accumulates regarding brain mechanisms/circuitry involved in neurofeedback and symptom changes, we forecast that EEG neurofeedback may be more commonly used without fMRI recording to augment psychotherapy and/or pharmacotherapy treatment for PTSD.

\section{Developing Precision Medicine Approaches to Treatment: Clinician Recommendations}

How can our findings inform clinicians working with patients who have experienced significant trauma? Just as there are many pathways to a PTSD diagnosis post-trauma, there is no "one size fits all” treatment for PTSD. Just as neuropsychological tests can be used by clinicians to estimate the degree and type of executive functioning deficits for an individual with PTSD (187), we posit that an assessment of EEG frequency and auditory/ visual ERP metrics of attention/working memory could provide important insights regarding impairments that could be targeted in personalized interventions. Notably, the current literature is not at the point in which findings can be used to directly inform treatment decisions or approaches with individual patients. However, there are several potential avenues in which EEG/ERP studies could soon have clinical relevance and thus, could be of top priority for future clinical research.

Our review of ERP studies suggest that some individuals with PTSD, particularly those experiencing hyperarousal, intrusions, and dissociation, show specific deficits in auditory working memory that could potentially be targeted by cognitive training similar to that employed by researchers to reduce 
auditory impairments in patients diagnosed with schizophrenia [e.g., Ref. (208)]. Furthermore, MMN amplitude, shown to be impaired as a function of PTSD, successfully indexes auditory improvements in schizophrenia patients after cognitive training (209); additional research is warranted to determine if auditory training can enhance attentional focus in PTSD patients, and if MMN amplitude change reflects hypothesized success in training. Individuals with PTSD, especially those exposed to combat or trauma specifically linked to auditory signals (e.g., bombs or explosions) should be first referred for hearing tests to determine whether basic auditory capacity is impaired and can be remedied.

On the whole, many patients with PTSD show attentional bias to visual stimuli relevant to the trauma they have experienced. As prolonged exposure therapy shows success in reducing PTSD symptoms [e.g., Ref. (210)], P2, P3, and LPP amplitude pre-, during-, and post-treatment may effectively track habituation to trauma and/or predict at the outset who would improve the most from exposure therapy. These ERP metrics may also predict or measure success of other treatments, including attentional bias modification therapy, which specifically targets exaggerated threat monitoring and significantly reduces PTSD risk after combat trauma (211). A subset of patients devote less neural resources to the processing of task-relevant visual stimuli, independent of trauma, reflected by small target P3b amplitudes; identifying individuals with this specific deficit and referring them to cognitive training enhancing visual attention may improve goal achievement and overall life functioning.

Identifying an individual's strengths and weaknesses with respect to attention, working memory, inhibition, and reward/ emotion processing using EEG/ERPs may determine what types of treatment are warranted; particular patients may benefit from more generalized cognitive training, brain stimulation, and/or neurofeedback in addition to trauma-focused therapies such as exposure.

\section{REFERENCES}

1. Goldstein RB, Smith SM, Chou SP, Saha TD, Jung J, Zhang H, et al. The epidemiology of DSM-5 posttraumatic stress disorder in the United States: results from the National Epidemiologic Survey on Alcohol and Related Conditions-III. Soc Psychiatry Psychiatr Epidemiol (2016) 51:1137-48. doi: 10.1007/s00127-016-1208-5

2. Colvonen PJ, Glassman LH, Crocker LD, Buttner MM, Orff H, Schiehser $\mathrm{DM}$, et al. Pretreatment biomarkers predicting PTSD psychotherapy outcomes: a systematic review. Neurosci Biobehav Rev (2017) 75:140-56. doi: 10.1016/j.neubiorev.2017.01.027

3. Fenster RJ, Lebois LAM, Ressler KJ, Suh J. Brain circuit dysfunction in posttraumatic stress disorder: from mouse to man. Nat Rev Neurosci (2018) 19:535-51. doi: 10.1038/s41583-018-0039-7

4. Hayes JP, Vanelzakker MB, Shin LM. Emotion and cognition interactions in PTSD: a review of neurocognitive and neuroimaging studies. Front Integr Neurosci (2012) 6:89. doi: 10.3389/fnint.2012.00089

5. Simmons AN, Matthews SC. Neural circuitry of PTSD with or without mild traumatic brain injury: a meta-analysis. Neuropharmacology (2012) 62:598606. doi: 10.1016/j.neuropharm.2011.03.016

6. Ball TM, Stein MB, Ramsawh HJ, Campbell-Sills L, Paulus MP. Single-subject anxiety treatment outcome prediction using functional neuroimaging. Neuropsychopharmacology (2014) 39:1254-61. doi: 10.1038/ npp. 2013.328

\section{CONCLUSIONS}

There is promise for designing interventions to more directly target EEG biomarkers related to PTSD and/or specific symptom dimensions to enhance clinical outcomes. Future clinical neuroscience research can build upon these results by: 1) further evaluating the clinical utility of CNV, MMN, P3a, P3b, and frontal/parietal alpha asymmetry as predictive and prognostic biomarkers of PTSD symptom course and treatment outcome; and 2) targeting biological and psychological processes involving frontoparietal attention networks in individuals suffering from PTSD-related symptoms.

\section{AUTHOR CONTRIBUTIONS}

$\mathrm{MB}$ and EE reviewed articles, compiled relevant data into tables, summarized main findings of each article, and provided feedback on versions of the manuscript. RA and VN provided feedback on figures, tables, interpretation of results, and multiple versions of the manuscript. JS checked data entered into tables, created the figures, wrote the first draft of the manuscript, and formatted the manuscript for journal submission.

\section{FUNDING}

This work was supported by The William K. Warren Foundation.

\section{ACKNOWLEDGMENTS}

Dr. Stewart wishes to thank students from the Research on Anxiety, Addiction, and Depression lab at Queens College who motivated this review.

7. American Psychiatric Association. Diagnostic and statistical manual of mental disorders (DSM-IV). 4th Edition ed. Washington DC: American Psychiatric Association (1994).

8. Wamser-Nanney R, Vandenberg BR. Empirical support for the definition of a complex trauma event in children and adolescents. J Trauma Stress (2013) 26:671-8. doi: 10.1002/jts.21857

9. American Psychiatric Association. Diagnostic and statistical manual of mental disorders $\left(D S M-5^{\circledR}\right)$. 5th Edition ed. Washington, DC: American Psychiatric Publications (2013). doi: 10.1176/appi.books.9780890425596

10. Sadeh N, Spielberg JM, Hayes JP. Impulsive responding in threat and reward contexts as a function of PTSD symptoms and trait disinhibition. J Anxiety Disord (2018) 53:76-84. doi: 10.1016/j.janxdis.2017.11.001

11. Sripada RK, Hoff R, Pfeiffer PN, Ganoczy D, Blow FC, Bohnert KM. Latent classes of PTSD symptoms in veterans undergoing residential PTSD treatment. Psychol Serv (2018) (Advance online publication). doi: 10.1037/ser0000284

12. Cuthbert BN. Translating intermediate phenotypes to psychopathology: the NIMH Research Domain Criteria. Psychophysiology (2014) 51:1205-6. doi: 10.1111/psyp. 12342

13. Hajcak G, Patrick CJ. Situating psychophysiological science within the Research Domain Criteria (RDoC) framework. Int J Psychophysiol (2015) 98:223-6. doi: 10.1016/j.ijpsycho.2015.11.001

14. Insel TR. The NIMH Research Domain Criteria (RDoC) Project: precision medicine for psychiatry. Am J Psychiatry (2014) 171:395-7. doi: 10.1176/ appi.ajp.2014.14020138 
15. FDA-NIH Biomarker Working Group. BEST (biomarkers, endpoints, and other tools) resource. (2016). https://www.ncbi.nlm.nih.gov/pubmed/27010052

16. Banducci AN, Bujarski SJ, Bonn-Miller MO, Patel A, Connolly KM. The impact of intolerance of emotional distress and uncertainty on veterans with co-occurring PTSD and substance use disorders. J Anxiety Disord (2016) 41:73-81. doi: 10.1016/j.janxdis.2016.03.003

17. Vujanovic AA, Bonn-Miller MO, Petry NM. Co-occurring posttraumatic stress and substance use: emerging research on correlates, mechanisms, and treatments-Introduction to the special issue. Psychol Addict Behav (2016) 30:713-9. doi: 10.1037/adb0000222

18. Post LM, Zöllner LA, Youngstrom E, Feeny NC. Understanding the relationship between co-occurring PTSD and MDD: symptom severity and affect. J Anxiety Disord (2011) 25:1123-30. doi: 10.1016/j. janxdis.2011.08.003

19. Bangasser DA, Valentino RJ. Sex differences in stress-related psychiatric disorders: neurobiological perspectives. Front Neuroendocrinol (2014) 35:303-19. doi: 10.1016/j.yfrne.2014.03.008

20. Weathers FW, Bovin MJ, Lee DJ, Sloan DM, Schnurr PP, Kaloupek DG, et al. The Clinician-Administered PTSD Scale for DSM-5 (CAPS-5): development and initial psychometric evaluation in military veterans. Psychol Assess (2018) 30:383-95. doi: 10.1037/pas0000486

21. First MB, Williams JB. SCID-5-CV: Structured Clinical Interview for DSM-5 Disorders: Clinician Version. Washington, DC: American Psychiatric Association Publishing (2016).

22. Blevins CA, Weathers FW, Davis MT, Witte TK, Domino JL. The Posttraumatic Stress Disorder Checklist for DSM-5 (PCL-5): development and initial psychometric evaluation. J Trauma Stress (2015) 28:489-98. doi: $10.1002 /$ jts.22059

23. Foa EB, McLean CP, Zang Y, Zhong J, Rauch S, Porter K, et al. Psychometric properties of the posttraumatic stress disorder symptom scale interview for DSM-5 (PSSI-5). Psychol Assess (2016) 28(10):1159. doi: 10.1037/ pas0000259

24. Blake DD, Weathers FW, Nagy LM, Kaloupek DG, Gusman FD, Charney DS, et al. The development of a Clinician-Administered PTSD Scale. J Trauma Stress (1995) 8:75-90. doi: 10.1007/BF02105408

25. Weathers FW, Keane TM, Davidson JR. Clinician-administered PTSD scale: a review of the first ten years of research. Depress Anxiety (2001) 13:132-56. doi: $10.1002 /$ da. 1029

26. Foa EB, Tolin DF. Comparison of the PTSD Symptom Scale-Interview Version and the Clinician-Administered PTSD scale. J Trauma Stress (2000) 13:181-91. doi: 10.1023/A:1007781909213

27. First MB, Spitzer RL, Gibbon MWJB, Williams JB. Structured clinical interview for DSM-IV axis I disorders. New York: New York State Psychiatric Institute (1995). doi: 10.1037/t07827-000

28. Weathers F, Huska J, Keane T. The PTSD checklist military version (PCL-M). Boston, MA: National Center for PTSD (1991).

29. Weathers FW, Litz BT, Herman D, Huska J, Keane T. The PTSD checklistcivilian version (PCL-C). Boston, MA: National Center for PTSD (1994). doi: 10.1037/t02622-000

30. Davidson JRT, Book SW, Colket JT, Tupler LA, Roth S, David D, et al. Assessment of a new self-rating scale for post-traumatic stress disorder. Psychol Med (1997) 27:153-60. doi: 10.1017/S0033291796004229

31. Weiss DS, Marmar CR. The Impact of Event Scale - Revised. In: Keane JWTM, editor. Assessing psychological trauma and PTSD. Guilford., New York: Guilford Press (1997). p. 399-411. doi: 10.1037/t12199-000

32. Blanchard EB, Jones-Alexander J, Buckley TC, Forneris CA. Psychometric properties of the PTSD Checklist (PCL). Behav Res Ther (1996) 34:669-73. doi: 10.1016/0005-7967(96)00033-2

33. Creamer M, Bell R, Failla S. Psychometric properties of the impact of event scale-revised. Behav Res Ther (2003) 41:1489-96. doi: 10.1016/j. brat.2003.07.010

34. Foa EB, Riggs DS, Dancu CV, Rothbaum BO. Reliability and validity of a brief instrument for assessing post-traumatic stress disorder. J Trauma Stress (1993) 6:459-73. doi: 10.1007/BF00974317

35. McFarlane A, Clark CR, Bryant RA, Williams LM, Niaura R, Paul RH, et al. The impact of early life stress on psychophysiological, personality and behavioral measures in 740 non-clinical subjects. J Integr Neurosci (2005) 4:27-40. doi: $10.1142 / \mathrm{S} 0219635205000689$
36. Bernstein DP, Stein JA, Newcomb MD, Walker E, Pogge D, Ahluvalia T, et al. Development and validation of a brief screening version of the Childhood Trauma Questionnaire. Child Abuse Negl (2003) 27:169-90. doi: 10.1016/ S0145-2134(02)00541-0

37. Walsh CA, MacMillan HL, Trocme N, Jamieson E, Boyle MH. Measurement of victimization in adolescence: development and validation of the Childhood Experiences of Violence Questionnaire. Child Abuse Negl (2008) 32:1037-57. doi: 10.1016/j.chiabu.2008.05.003

38. Keane TM, Caddell JM, Taylor KL. Mississippi Scale for Combat-Related Posttraumatic Stress Disorder: three studies in reliability and validity. J Consult Clin Psychol (1988) 56:85-90. doi: 10.1037//0022-006X.56.1.85

39. Keane TM, Fairbank JA, Caddell JM, Zimering RT, Taylor KL, Mora CA. Clinical evaluation of a measure to assess combat exposure. J Consult Clin Psychol (1989) 1:53-5. doi: 10.1037/1040-3590.1.1.53

40. Cicchetti D, Curtis WJ. Multilevel perspectives on pathways to resilient functioning. Dev Psychopathol (2007) 19:627-9. doi: 10.1017/ S0954579407000314

41. Miskovic V, Schmidt LA, Georgiades K, Boyle M, MacMillan HL. Stability of resting frontal electroencephalogram (EEG) asymmetry and cardiac vagal tone in adolescent females exposed to child maltreatment. Dev Psychobiol (2009) 51:474-87. doi: 10.1002/dev.20387

42. Rabe S, Beauducel A, Zöllner T, Maercker A, Karl A. Regional brain electrical activity in posttraumatic stress disorder after motor vehicle accident. J Abnorm Psychol (2006) 115:687-98. doi: 10.1037/0021-843X. 115.4.687

43. Meyer T, Quaedflieg C, Weijland K, Schruers K, Merckelbach H, Smeets T. Frontal EEG asymmetry during symptom provocation predicts subjective responses to intrusions in survivors with and without PTSD. Psychophysiology (2018) 55:e12779. doi: 10.1111/psyp.12779

44. Kemp M, Drummond P, McDermott B. A wait-list controlled pilot study of eye movement desensitization and reprocessing (EMDR) for children with post-traumatic stress disorder (PTSD) symptoms from motor vehicle accidents. Clin Child Psychol Psychiatry (2010) 15:5-25. doi: $10.1177 / 1359104509339086$

45. Metzger LJ, Paige SR, Carson MA, Lasko NB, Paulus LA, Pitman RK, et al. PTSD arousal and depression symptoms associated with increased rightsided parietal EEG asymmetry. J Abnorm Psychol (2004) 113:324-9. doi: 10.1037/0021-843X.113.2.324

46. Veltmeyer MD, McFarlane AC, Bryant RA, Mayo T, Gordon E, Clark CR. Integrative assessment of brain function in PTSD: brain stability and working memory. J Integr Neurosci (2006) 5:123-38. doi: 10.1142/ S0219635206001057

47. Wahbeh H, Oken BS. Peak high-frequency HRV and peak alpha frequency higher in PTSD. Appl Psychophysiol Biofeedback (2013) 38:57-69. doi: 10.1007/s10484-012-9208-z

48. Lee SH, Yoon S, Kim JI, Jin SH, Chung CK. Functional connectivity of resting state EEG and symptom severity in patients with post-traumatic stress disorder. Prog Neuropsychopharmacol Biol Psychiatry (2014) 51:51-7. doi: 10.1016/j.pnpbp.2014.01.008

49. Metzger LJ, Carson MA, Paulus LA, Lasko NB, Paige SR, Pitman RK, et al. Event-related potentials to auditory stimuli in female Vietnam nurse veterans with posttraumatic stress disorder. Psychophysiology (2002) 39:49-63. doi: 10.1017/S0048577202001002

50. Felmingham KL, Bryant RA, Kendall C, Gordon E. Event-related potential dysfunction in posttraumatic stress disorder: the role of numbing. Psychiatry Res (2002) 109:171-9. doi: 10.1016/S0165-1781(02)00003-3

51. Lewine JD, Thoma RJ, Provencal SL, Edgar C, Miller GA, Canive JM. Abnormal stimulus-response intensity functions in posttraumatic stress disorder: an electrophysiological investigation. Am J Psychiatry (2002) 159:1689-95. doi: 10.1176/appi.ajp.159.10.1689

52. Metzger LJ, Clark CR, McFarlane AC, Veltmeyer MD, Lasko NB, Paige SR, et al., Event-related potentials to auditory stimuli in monozygotic twins discordant for combat: sssociation with PTSD. Psychophysiology (2009) 46(1):172-8. doi: 10.1111/j.1469-8986.2008.00720.x

53. Falconer EM, Felmingham KL, Allen A, Clark CR, McFarlane AC, Williams $\mathrm{LM}$, et al. Developing an integrated brain, behavior and biological response profile in posttraumatic stress disorder (PTSD). J Integr Neurosci (2008) 7:439-56. doi: 10.1142/S0219635208001873 
54. Araki T, Kasai K, Yamasue H, Kato N, Kudo N, Ohtani T, et al. Association between lower P300 amplitude and smaller anterior cingulate cortex volume in patients with posttraumatic stress disorder: a study of victims of Tokyo subway sarin attack. NeuroImage (2005) 25:43-50. doi: 10.1016/j. neuroimage.2004.11.039

55. Boudarene M, Timsit-Berthier M. Interest of events-related potentials in assessment of posttraumatic stress disorder. Ann NY Acad Sci (1997) 821:494-8. doi: 10.1111/j.1749-6632.1997.tb48314.x

56. Charles G, Hansenne M, Ansseau M, Pitchot W, Machowski R, Schittecatte M, et al. P300 in posttraumatic stress disorder. Neuropsychobiol (1995) 32:72-4. doi: 10.1159/000119216

57. Metzger LJ, Orr SP, Lasko NB, Pitman RK. Auditory event-related potentials to tone stimuli in combat-related posttraumatic stress disorder. Biol Psychiatry (1997) 42:1006-15. doi: 10.1016/S0006-3223(97)00138-8

58. Bae KY, Kim DW, Im CH, Lee SH. Source imaging of P300 auditory evoked potentials and clinical correlations in patients with posttraumatic stress disorder. Prog Neuropsychopharmacol Biol Psychiatry (2011) 35:1908-17. doi: 10.1016/j.pnpbp.2011.08.002

59. Kimura M, Ueda M, Takeda Y, Sugimoto F, Katayama J. Aftermath of 3/11: earthquakes and involuntary attentional orienting to sudden ambient sounds. Biol Psychol (2013) 94:419-25. doi: 10.1016/j.biopsycho.2013.08.008

60. Kimura M, Sugimoto F, Ueda M, Takeda Y, Katayama JI. Aftermath of 3/11: a pilot study on the relationship between indirect exposure to earthquakes and auditory attention. Psychologia (2015) 58:27-5. doi: 10.2117/psysoc.2015.27

61. Bangel KA, van Buschbach S, Smit DJA, Mazaheri A, Olff M. Aberrant brain response after auditory deviance in PTSD compared to trauma controls: an EEG study. Sci Rep (2017) 7:16596. doi: 10.1038/s41598-017-16669-8

62. Morgan CA, Grillon C. Abnormal mismatch negativity in women with sexual assault-related posttraumatic stress disorder. Biol Psychiatry (1999) 45:827-32. doi: 10.1016/S0006-3223(98)00194-2

63. Schaefer LM, Nooner KB. Brain function associated with cooccurring trauma and depression symptoms in college students. J Aggress Maltreat Trauma (2017) 2(2):175-90. doi: 10.1080/10926771.2016.1272656

64. Zhang Y, Kong F, Han L, Najam Ul Hasan A, Chen H. Attention bias in earthquake-exposed survivors: an event-related potential study. Int J Psychophysiol (2014) 94:358-64. doi: 10.1016/j.ijpsycho.2014.09.004

65. Zuj DV, Felmingham KL, Palmer MA, Lawrence-Wood E, Van Hooff M, Lawrence AJ, et al. Neural activity and emotional processing following military deployment: effects of mild traumatic brain injury and posttraumatic stress disorder. Brain Cogn (2017) 118:19-26. doi: 10.1016/j. bandc.2017.07.001

66. MacNamara A, Post D, Kennedy AE, Rabinak CA, Phan KL. Electrocortical processing of social signals of threat in combat-related post-traumatic stress disorder. Biol Psychol (2013) 94:441-9. doi: 10.1016/j.biopsycho.2013.08.009

67. Wessa M, Karl A, Flor H. Central and peripheral psychophysiological responses to trauma-related cues in subclinical posttraumatic stress disorder: a pilot study. Exp Brain Res (2005) 167:56-65. doi: 10.1007/ s00221-005-0007-0

68. Stanford MS, Vasterling JJ, Mathias CW, Constans JI, Houston RJ. Impact of threat relevance on P3 event-related potentials in combat-related posttraumatic stress disorder. Psychiatry Res (2001) 102:125-37. doi: 10.1016/ S0165-1781(01)00236-0

69. Veltmeyer MD, Clark CR, McFarlane AC, Moores KA, Bryant RA, Gordon E. Working memory function in post-traumatic stress disorder: an eventrelated potential study. Clin Neurophysiol (2009) 120:1096-106. doi: 10.1016/j.clinph.2009.03.024

70. Gilmore CS, Marquardt CA, Kang SS, Sponheim SR. Reduced P3b brain response during sustained visual attention is associated with remote blast mTBI and current PTSD in U.S. military veterans. Behav Brain Res (2018) 340:174-82. doi: 10.1016/j.bbr.2016.12.002

71. Shah D, Cameron C, Smith D, Jaworska N, Blais C, Fisher D, et al. An electrophysiological investigation of attentional bias in a PTSD population. Psychol Trauma (2018) 10:523-32. doi: 10.1037/tra0000339

72. Bleich AV, Attias J, Furman V. Effect of repeated visual traumatic stimuli on the event related P3 brain potential in post-traumatic stress disorder. Int J Neurosci (1996) 85:45-55. doi: 10.3109/00207459608986350

73. Tillman GD, Calley CS, Green TA, Buhl VI, Biggs MM, Spence JS, et al. Visual event-related potentials as markers of hyperarousal in Gulf War illness: evidence against a stress-related etiology. Psychiatry Res (2013) 211:257-67. doi: 10.1016/j.pscychresns.2012.08.004

74. Wang C, Costanzo ME, Rapp PE, Darmon D, Bashirelahi K, Nathan DE, et al. Identifying electrophysiological prodromes of post-traumatic stress disorder: results from a pilot study. Front Psychiatry (2017) 8:71. doi: 10.3389/fpsyt.2017.00071

75. Tso IF, Chiu PH, King-Casas BR, Deldin PJ. Alterations in affective processing of attack images following September 11, 2001. J Trauma Stress (2011) 24:538-45. doi: 10.1002/jts.20678

76. Wang C, Rapp P, Darmon D, Trongnetrpunya A, Costanzo ME, Nathan DE, et al. Utility of P300 ERP in monitoring post-trauma mental health: a longitudinal study in military personnel returning from combat deployment. J Psychiatr Res (2018) 101:5-13. doi: 10.1016/j. jpsychires.2018.02.027

77. Saar-Ashkenazy R, Shalev H, Kanthak MK, Guez J, Friedman A, Cohen JE. Altered processing of visual emotional stimuli in posttraumatic stress disorder: an event-related potential study. Psychiatry Res (2015) 233:165-74. doi: 10.1016/j.pscychresns.2015.05.015

78. DiGangi JA, Burkhouse KL, Aase DM, Babione JM, Schroth C, Kennedy $\mathrm{AE}$, et al. An electrocortical investigation of emotional face processing in military-related posttraumatic stress disorder. J Psychiatr Res (2017) 92:1328. doi: 10.1016/j.jpsychires.2017.03.013

79. DiGangi JA, Kujawa A, Aase DM, Babione JM, Schroth C, Levy DM, et al. Affective and cognitive correlates of PTSD: electrocortical processing of threat and perseverative errors on the WCST in combat-related PTSD. Prog Neuropsychopharmacol Biol Psychiatry (2017) 75:63-9. doi: 10.1016/j. pnpbp.2017.01.004

80. Kujawa A, Hajcak G, Danzig AP, Black SR, Bromet EJ, Carlson GA, et al. Neural reactivity to emotional stimuli prospectively predicts the impact of a natural disaster on psychiatric symptoms in children. Biol Psychiatry (2016) 80:381-9. doi: 10.1016/j.biopsych.2015.09.008

81. Lobo I, David IA, Figueira I, Campagnoli RR, Volchan E, Pereira MG, et al. Brain reactivity to unpleasant stimuli is associated with severity of posttraumatic stress symptoms. Biol Psychol (2014) 103:233-41.

82. Duan H, Wang L, Fernandez G, Zhang K, Wu J. Increased anticipatory contingent negative variation in posttraumatic stress disorder. Biol Psychol (2016) 117:80-8. doi: 10.1016/j.biopsycho.2016.03.004

83. Chu DA, Bryant RA, Gatt JM, Harris AW. Failure to differentiate between threat-related and positive emotion cues in healthy adults with childhood interpersonal or adult trauma. J Psychiatr Res (2016) 78:31-41. doi: 10.1016/j. jpsychires.2016.03.006

84. Trujillo SP, Valencia S, Trujillo N, Ugarriza JE, Rodriguez MV, Rendon J, et al. Atypical modulations of N170 component during emotional processing and their links to social behaviors in ex-combatants. Front Hum Neurosci (2017) 11:244. doi: 10.3389/fnhum.2017.00244

85. Chu DA, Bryant RA, Gatt JM, Harris AW. Cumulative childhood interpersonal trauma is associated with reduced cortical differentiation between threat and non-threat faces in posttraumatic stress disorder adults. Aust NZJ Psychiatry (2019) 53:48-58. doi: 10.1177/2F0004867418761578

86. Kimble M, Sripad A, Fowler R, Sobolewski S, Fleming K. Negative world views after trauma: neurophysiological evidence for negative expectancies. Psychol Trauma (2018) 10:576-84. doi: 10.1037/tra0000324

87. Cui H, Liu X, Chen G, Shan M, Jia Y. An event-related potentials study on the attention function of posttraumatic stress disorder. Int J Clin Exp Med (2015) 8:10885-92.

88. Wei DT, Qiu J, Du X, Luo YJ. Emotional arousal to negative information after traumatic experiences: an event-related brain potential study. Neuroscience (2011) 192:391-7. doi: 10.1016/j.neuroscience.2011.06.055

89. Wu J, Ge Y, Shi Z, Duan X, Wang L, Sun X, et al. Response inhibition in adolescent earthquake survivors with and without posttraumatic stress disorder: a combined behavioral and ERP study. Neurosci Lett (2010) 486:117-21. doi: 10.1016/j.neulet.2010.07.040

90. Covey TJ, Shucard JL, Violanti JM, Lee J, Shucard DW. The effects of exposure to traumatic stressors on inhibitory control in police officers: a dense electrode array study using a Go/NoGo continuous performance task. Int J Psychophysiol (2013) 87:363-75. doi: 10.1016/j.ijpsycho.2013.03.009

91. Shucard JL, McCabe DC, Szymanski H. An event-related potential study of attention deficits in posttraumatic stress disorder during auditory and visual 
Go/NoGo continuous performance tasks. Biol Psychol (2008) 79:223-33. doi: 10.1016/j.biopsycho.2008.05.005

92. Wu J, Yuan Y, Cao C, Zhang K, Wang L, Zhang L. The relationship between response inhibition and posttraumatic stress symptom clusters in adolescent earthquake survivors: an event-related potential study. Sci Rep (2015) 5:8844. doi: $10.1038 /$ srep08844

93. Khan NI, Burkhouse KL, Lieberman L, Gorka SM, DiGangi JA, Schroth C, et al. Individual differences in combat experiences and error-related brain activity in OEF/OIF/OND veterans. Int J Psychophysiol (2018) 129:52-7. doi: 10.1016/j.jpsycho.2018.04.011

94. Meyer A, Danielson CK, Danzig AP, Bhatia V, Black SR, Bromet E, et al. Neural biomarker and early temperament predict increased internalizing symptoms after a natural disaster. J Am Acad Child Adolesc Psychiatry (2017) 56:410-6. doi: 10.1016/j.jaac.2017.02.005

95. Melara RD, Ruglass LM, Fertuck EA, Hien DA. Regulation of threat in post-traumatic stress disorder: associations between inhibitory control and dissociative symptoms. Biol Psychol (2018) 133:89-98. doi: 10.1016/j. biopsycho.2018.01.017

96. Fitzgerald JM, MacNamara A, Kennedy AE, Rabinak CA, Rauch SA, Liberzon I, et al. Individual differences in cognitive reappraisal use and emotion regulatory brain function in combat-exposed veterans with and without PTSD. Depress Anxiety (2017) 34:79-88. doi: 10.1002/da.22551

97. Begić D, Hotujac L, Jokić-Begić N. Electroencephalographic comparison of veterans with combat-related post-traumatic stress disorder and healthy subjects. Int J Psychophysiol (2001) 40:167-72. doi: 10.1016/ S0167-8760(00)00153-7

98. Cowdin N, Kobayashi I, Mellman TA. Theta frequency activity during rapid eye movement (REM) sleep is greater in people with resilience versus PTSD. Exp Brain Res (2014) 232:1479-85. doi: 10.1007/s00221-014-3857-5

99. Ehlers CL, Hurst S, Phillips E, Gilder DA, Dixon M, Gross A, et al. Electrophysiological responses to affective stimuli in American Indians experiencing trauma with and without PTSD. Ann NY Acad Sci (2006) 1071:125-36. doi: 10.1196/annals.1364.011

100. Hostinar CE, Davidson RJ, Graham EK, Mroczek DK, Lachman ME, Seeman T, et al. Frontal brain asymmetry, childhood maltreatment, and low-grade inflammation at midlife. Psychoneuroendocrinology (2017) 75:152-63. doi: 10.1016/j.psyneuen.2016.10.026

101. Imperatori C, Farina B, Quintiliani MI, Onofri A, Castelli Gattinara P, Lepore $M$, et al. Aberrant EEG functional connectivity and EEG power spectra in resting state post-traumatic stress disorder: a sLORETA study. Biol Psychol (2014) 102:10-17. doi: 10.1016/j.biopsycho.2014.07.011

102. Rabe S, Zöllner T, Maercker A, Karl A. Neural correlates of posttraumatic growth after severe motor vehicle accidents. J Consult Clin Psychol (2006) 74:880-6. doi: 10.1037/0022-006X.74.5.880

103. Shankman SA, Silverstein SM, Williams LM, Hopkinson PJ, Kemp AH, Felmingham KL, et al. Resting electroencephalogram asymmetry and posttraumatic stress disorder. J Trauma Stress (2008) 21:190-8. doi: 10.1002/ jts.20319

104. Tang A, Miskovic V, Lahat A, Tanaka M, MacMillan H, Van Lieshout RJ, et al. Trajectories of resting frontal brain activity and psychopathology in female adolescents exposed to child maltreatment. Dev Psychobiol (2018) 60:67-77. doi: $10.1002 /$ dev. 21585

105. Todder D, Levine J, Abujumah A, Mater M, Cohen H, Kaplan Z. The quantitative electroencephalogram and the low-resolution electrical tomographic analysis in posttraumatic stress disorder. Clin EEG Neurosci (2012) 43:48-53. doi: 10.1177/1550059411428716

106. Blomhoff S, Reinvang I, Malt UF. Event-related potentials to stimuli with emotional impact in posttraumatic stress patients. Biol Psychiatry (1998) 44:1045-53 doi: 10.1016/S0006-3223(98)00058-4

107. Felmingham KL, Rennie C, Gordon E, Bryant RA. Autonomic and cortical reactivity in acute and chronic posttraumatic stress. Biol Psychol (2012) 90:224-7. doi: 10.1016/j.biopsycho.2012.03.011

108. Galletly C, Clark CR, McFarlane AC, Weber DL. Working memory in posttraumatic stress disorder-an event-related potential study. J. Trauma Stress (2001) 14:295-309. doi: 10.1023/A:1011112917797

109. Ge Y, Wu J, Sun X, Zhang K. Enhanced mismatch negativity in adolescents with posttraumatic stress disorder (PTSD). Int J Psychophysiol (2011) 79:231-5. doi: 10.1016/j.ijpsycho.2010.10.012
110. Hall T, Galletly C, Clark CR, Veltmeyer M, Metzger LJ, Gilbertson MW, et al. The relationship between Hippocampal asymmetry and working memory processing in combat-related PTSD - a monozygotic twin study. Biol Mood Anxiety Disord (2012) 2:21. doi: 10.1186/2045-5380-2-21

111. Kimble M, Kaloupek D, Kaufman M, Deldin P. Stimulus novelty differentially affects attentional allocation in PTSD. Biol Psychiatry (2000) 47:880-90. doi: 10.1016/S0006-3223(99)00258-9

112. Lamprecht F, Kohnke C, Lempa W, Sack M, Matzke M, Munte TF. Eventrelated potentials and EMDR treatment of post-traumatic stress disorder. Neurosci Res (2004) 49:267-72. doi: 10.1016/j.neures.2004.02.013

113. McPherson WB, Newton JE, Ackerman P, Oglesby DM, Dykman RA. An event-related brain potential investigation of PTSD and PTSD symptoms in abused children. Integr Physiol Behav Sci (1997) 32:31-42. doi: 10.1007/ BF02688611

114. Menning H, Renz A, Seifert J, Maercker A. Reduced mismatch negativity in posttraumatic stress disorder: a compensatory mechanism for chronic hyperarousal? Int J Psychophysiol (2008) 68:27-34. doi: 10.1016/j. ijpsycho.2007.12.003

115. Neylan TC, Jasiukaitis PA, Lenoci M, Scott JC, Metzler TJ, Weiss DS, et al. Temporal instability of auditory and visual event-related potentials in posttraumatic stress disorder. Biol Psychiatry (2003) 53:216-25. doi: 10.1016/ S0006-3223(02)01450-6

116. Attias J, Bleich A, Furman V, Zinger Y. Event-related potentials in posttraumatic stress disorder of combat origin. Biol Psychiatry (1996) 40:373-81. doi: 10.1016/0006-3223(95)00419-X

117. Grasso DJ, Simons RF. Electrophysiological responses to threat in youth with and without Posttraumatic Stress Disorder. Biol Psychol (2012) 90:88-96. doi: 10.1016/j.biopsycho.2012.02.015

118. Honzel N, Justus T, Swick D. Posttraumatic stress disorder is associated with limited executive resources in a working memory task. Cogn Affect Behav Neurosci (2014) 14:792-804. doi: 10.3758/s13415-013-0219-x

119. Kessel EM, Nelson BD, Kujawa A, Hajcak G, Kotov R, Bromet EJ, et al. Hurricane Sandy exposure alters the development of neural reactivity to negative stimuli in children. Child Dev (2018) 89:339-48. doi: 10.1111/ cdev. 12691

120. Kimble M, Batterink L, Marks E, Ross C, Fleming K. Negative expectancies in posttraumatic stress disorder: neurophysiological (N400) and behavioral evidence. J Psychiatr Res (2012) 46:849-55. doi: 10.1016/j. jpsychires.2012.03.023

121. Klimova A, Bryant RA, Williams LM, Felmingham KL. Dysregulation in cortical reactivity to emotional faces in PTSD patients with high dissociation symptoms. Eur J Psychotraumatol (2013) 4:20430. doi: 10.3402/ejpt.v4i0.20430

122. Kounios J, Litz B, Kaloupek D, Riggs D, Knight J, Weathers F, et al. Electrophysiology of combat-related PTSD. Ann NY Acad Sci (1997) 821:504-7. doi: 10.1111/j.1749-6632.1997.tb48316.x

123. Shu IW, Onton JA, Prabhakar N, O'Connell RM, Simmons AN, Matthews SC. Combat veterans with PTSD after mild TBI exhibit greater ERPs from posterior-medial cortical areas while appraising facial features. $J$ Affect Disord (2014) 155:234-40. doi: 10.1016/j.jad.2013.06.057

124. Sokhadze E, Singh S, Stewart C, Hollifield M, El-Baz A, Tasman A. Attentional bias to drug- and stress-related pictorial cues in cocaine addiction comorbid with PTSD. J Neurother (2008) 12:205-25. doi: 10.1080/ 10874200802502185

125. Wessa M, Jatzko A, Flor H. Retrieval and emotional processing of traumatic memories in posttraumatic stress disorder: peripheral and central correlates. Neuropsychologia (2006) 44:1683-96. doi: 10.1016/j. neuropsychologia.2006.03.024

126. Yun X, Li W, Qiu J, Jou J, Wei D, Tu S, et al. Neural mechanisms of subliminal priming for traumatic episodic memory: an ERP study. Neurosci Lett (2011) 498:10-4. doi: 10.1016/j.neulet.2011.04.040

127. Zhang Y, Kong F, Hasan AN, Jackson T, Chen H. Recognition memory bias in earthquake-exposed survivors: a behavioral and ERP study. Neuropsychobiology (2015) 71:70-5. doi: 10.1159/000369023

128. Chen X, Wei D, Dupuis-Roy N, Du X, Qiu J, Zhang Q. Neural correlates of the modified Stroop effect in post-traumatic stress disorder: an eventrelated potential study. Neuroreport (2012) 23:1035-8. doi: 10.1097/ WNR.0b013e32835a989e 
129. Gorka SM, Lieberman L, Shankman SA, Phan KL. Association between neural reactivity and startle reactivity to uncertain threat in two independent samples. Psychophysiology (2017) 54:652-62. doi: 10.1111/psyp.12829

130. Lackner CL, Santesso DL, Dywan J, O'Leary DD, Wade TJ, Segalowitz SJ. Adverse childhood experiences are associated with self-regulation and the magnitude of the error-related negativity difference. Biol Psychol (2018) 132:244-51. doi: 10.1016/j.biopsycho.2018.01.006

131. Lieberman L, Gorka SM, Funkhouser CJ, Shankman SA, Phan KL. Impact of posttraumatic stress symptom dimensions on psychophysiological reactivity to threat and reward. J Psychiatr Res (2017) 92:55-63. doi: 10.1016/j. jpsychires.2017.04.002

132. Rabinak CA, Holman A, Angstadt M, Kennedy AE, Hajcak G, Phan KL. Neural response to errors in combat-exposed returning veterans with and without post-traumatic stress disorder: a preliminary event-related potential study. Psychiatry Res (2013) 213:71-8. doi: 10.1016/j.pscychresns. 2013.01.002

133. Qiu J, Su Y, Li H, Wei D, Tu S, Zhang Q. How personal earthquake experience impacts on the Stroop interference effect: an event-related potential study. Psychophysiology (2010) 47:995-1001. doi: 10.1111/ j.1469-8986.2010.01017.x

134. Shu IW, Onton JA, O’Connell RM, Simmons AN, Matthews SC. Combat veterans with comorbid PTSD and mild TBI exhibit a greater inhibitory processing ERP from the dorsal anterior cingulate cortex. Psychiatry Res (2014) 224:58-66. doi: 10.1016/j.pscychresns.2014.07.010

135. Swick D, Honzel N, Turken U. Intact error monitoring in combat Veterans with post-traumatic stress disorder. Psychiatry Res (2015) 234:227-38. doi: 10.1016/j.pscychresns.2015.09.016

136. Fitzgerald JM, Gorka SM, Kujawa A, DiGangi JA, Proescher E, Greenstein JE, et al. Neural indices of emotional reactivity and regulation predict course of PTSD symptoms in combat-exposed veterans. Prog Neuropsychopharmacol Biol Psychiatry (2018) 82:255-62. doi: 10.1016/j. pnpbp.2017.11.005

137. Li JZ, Gui DY, Feng CL, Wang WZ, Du BQ, Gan T, et al. Victims' time discounting 2.5 years after the Wenchuan earthquake: an ERP study. PLoS One (2012) 7:e40316. doi: 10.1371/journal.pone.0040316

138. Pechtel P, Dutra SJ, Goetz EL, Pizzagalli DA. Blunted reward responsiveness in remitted depression. J Psychiatr Res (2013) 47:1864-9. doi: 10.1016/j. jpsychires.2013.08.011

139. Newson JJ, Thiagarajan TC. EEG frequency bands in psychiatric disorders: a review of resting state studies. Front Hum Neurosci (2018) 12:521. doi: 10.1111/j.1469-8986.2011.01293.x

140. Davidson RJ. What does the prefrontal cortex "do" in affect: perspectives on frontal EEG asymmetry research. Biol Psychol (2004) 67:219-33. doi: 10.1016/j.biopsycho.2004.03.008

141. Hagemann D, Hewig J, Seifert J, Naumann E, Bartussek D. The latent state-trait structure of resting EEG asymmetry: replication and extension. Psychophysiology (2005) 42:740-52. doi: 10.1111/j.1469-8986.2005.00367.x

142. Knyazev GG. EEG delta oscillations as a correlate of basic homeostatic and motivational processes. Neurosci Biobehav Rev (2012) 36:677-95. doi: 10.1016/j.neubiorev.2011.10.002

143. Güntekin B, Başar E. Review of evoked and event-related delta responses in the human brain. Int J Psychophysiol (2016) 103:43-52. doi: 10.1016/j. ijpsycho.2015.02.001

144. Harmony T. The functional significance of delta oscillations in cognitive processing. Front Integr Neurosci (2013) 7:83. doi: 10.3389/fnint.2013.00083

145. Gevins A, Smith ME, McEvoy L, Yu D. High-resolution EEG mapping of cortical activation related to working memory: effects of task difficulty, type of processing, and practice. Cereb Cortex (1997) 7:374-85 doi: 10.1093/ cercor/7.4.374

146. Korotkova T, Ponomarenko A, Monaghan CK, Poulter SL, Cacucci F, Wills $\mathrm{T}$, et al. Reconciling the different faces of hippocampal theta: the role of theta oscillations in cognitive, emotional and innate behaviors. Neurosci Biobehav Rev (2018) 85:65-80. doi: 10.1016/j.neubiorev.2017.09.004

147. Roux F, Uhlhaas PJ. Working memory and neural oscillations: alpha-gamma versus theta-gamma codes for distinct WM information? Trends Cogn Sci (2014) 18:16-25. doi: 10.1016/j.tics.2013.10.010

148. Engel AK, Fries P. Beta-band oscillations-signaling the status quo? Curr Opin Neurobiol (2010) 20:156-65. doi: 10.1016/j.conb.2010.02.015
149. Herrmann CS, Kaiser J. EEG gamma-band responses reflect human behavior: an overview. Int J Psychophysiol (2011) 79:1-2. doi: 10.1016/j. ijpsycho.2010.10.001

150. Klimesch W. Alpha-band oscillations, attention, and controlled access to stored information. Trends Cogn Sci (2012) 16:606-17. doi: 10.1016/j. tics.2012.10.007

151. Allen JJ, Coan JA, Nazarian M. Issues and assumptions on the road from raw signals to metrics of frontal EEG asymmetry in emotion. Biol Psychol (2004) 67:183-218. doi: 10.1016/j.biopsycho.2004.03.007

152. Smith EE, Reznik SJ, Stewart JL, Allen JJ. Assessing and conceptualizing frontal EEG asymmetry: an updated primer on recording, processing, analyzing, and interpreting frontal alpha asymmetry. Int J Psychophysiol (2017) 111:98-114. doi: 10.1016/j.ijpsycho.2016.11.005

153. Harmon-Jones E, Gable PA, Peterson CK. The role of asymmetric frontal cortical activity in emotion-related phenomena: a review and update. Biol Psychol (2010) 84:451-62. doi: 10.1016/j.biopsycho.2009.08.010

154. Stewart JL, Bismark AW, Towers DN, Coan JA, Allen JJ. Resting frontal EEG asymmetry as an endophenotype for depression risk: sex-specific patterns of frontal brain asymmetry. J Abnorm Psychol (2010) 119:502-12. doi: 10.1037/ a0019196

155. Coan JA, Allen JJ, McKnight PE. A capability model of individual differences in frontal EEG asymmetry. Biol Psychol (2006) 72:198-207. doi: 10.1016/j. biopsycho.2005.10.003

156. Stewart JL, Coan JA, Towers DN, Allen JJ. Resting and task-elicited prefrontal EEG alpha asymmetry in depression: Support for the capability model. Psychophysiology (2014) 51:446-55. doi: 10.1111/psyp.12191

157. Bruder GE, Stewart JW, McGrath PJ. Right brain, left brain in depressive disorders: clinical and theoretical implications of behavioral, electrophysiological and neuroimaging findings. Neurosci Biobehav Rev (2017) 78:178-91. doi: 10.1016/j.neubiorev.2017.04.021

158. Stewart JL, Towers DN, Coan JA, Allen JJ. The oft-neglected role of parietal EEG asymmetry and risk for major depressive disorder. Psychophysiology (2011) 48:82-95. doi: 10.1111/j.1469-8986.2010.01035.x

159. Khanna A, Pascual-Leone A, Michel CM, Farzan F. Microstates in restingstate EEG: current status and future directions. Neurosci Biobehav Rev (2015) 49:105-13. doi: 10.1016/j.neubiorev.2014.12.010

160. Yuan H, Phillips R, Wong CK, Zotev V, Misaki M, Wurfel B, et al. Tracking resting state connectivity dynamics in veterans with PTSD. NeuroImage: Clinical (2018) 19:260-70. doi: 10.1016/j.nicl.2018.04.014

161. Kim J, Chae JH, Ko HK, Latchoumane CFV, Banerjee A, Mandell DJ, et al. Hemispheric asymmetry in non-linear interdependence of EEG in posttraumatic stress disorder. Psychiatry Clin Neurosci (2012) 66:87-96. doi: 10.1111/j.1440-1819.2011.02300.x

162. Chae JH, Jeong J, Peterson BS, Kim DJ, Bahk WM, Jun TY, et al. Dimensional complexity of the EEG in patients with posttraumatic stress disorder. Psychiatry Res Neuroimaging (2004) 131:79-89. doi: 10.1016/j. pscychresns.2003.05.002

163. Shim M, Im CH, Lee SH. Disrupted cortical brain network in post-traumatic stress disorder patients: a resting-state electroencephalographic study. Transl Psychiatry (2017) 7:e1231. doi: 10.1038/tp.2017.200

164. Fabiani M, Gratton G, Federmeier KD, Cacioppo JT, Tassinary LG, Berntson GG. Event-related brain potentials: methods, theory, and applications. In: Handbook of Psychophysiology. Cambridge University Press (2000). p. 53-84.

165. Polich J. Updating P300: an integrative theory of P3a and P3b. Clin Neurophysiol (2007) 118:2128-48.

166. Dunn BR, Dunn DA, Languis M, Andrews D. The relation of ERP components to complex memory processing. Brain Cogn (1998) 36:355-76. doi: 10.1016/j.clinph.2007.04.019

167. Paulmann S, Pell MD. Facial expression decoding as a function of emotional meaning status: ERP evidence. Neuroreport (2009) 20:1603-8. doi: 10.1006/ brcg.1998.0998

168. Downes M, Bathelt J, De Haan M. Event-related potential measures of executive functioning from preschool to adolescence. Dev Med Child Neurol. (2017) 59:581-90. doi: 10.1097/WNR.0b013e3283320e3f

169. Hugdahl K. Psychophysiology: The mind-body perspective. Cambridge, Mass: Harvard University Press (1995). doi: 10.1111/dmcn.13395

170. Comerchero MD, Polich J. P3a and P3b from typical auditory and visual stimuli. Clin Neurophysiol (1999) 110:24-30. 
171. Brown SB, van Steenbergen H, Band GP, de Rover M, Nieuwenhuis S. Functional significance of the emotion-related late positive potential. Front Hum Neurosci (2012) 6:33. doi: 10.1016/S0168-5597(98)00033-1

172. Liu Y, Huang H, McGinnis-Deweese M, Keil A, Ding M. Neural substrate of the late positive potential in emotional processing. J Neurosci (2012) 32:14563-72. doi: 10.3389/fnhum.2012.00033

173. Eimer M. The face-sensitivity of the n170 component. Front Hum Neurosci (2011) 5:119. doi: 10.1523/JNEUROSCI.3109-12.2012

174. Lobo I, Portugal LC, Figueira I, Volchan E, David I, Garcia Pereira M, et al. EEG correlates of the severity of posttraumatic stress symptoms: a systematic review of the dimensional PTSD literature. J Affect Disord (2015) 183:21020. doi: 10.3389/fnhum.2011.00119

175. Donchin E, Smith DB. The contingent negative variation and the late positive wave of the average evoked potential. Electroencephalogr Clin Neurophysiol (1970) 29:201-3. doi: 10.1016/j.jad.2015.05.015

176. Hamano T, Luders HO, Ikeda A, Collura TF, Comair YG, Shibasaki H. The cortical generators of the contingent negative variation in humans: a study with subdural electrodes. Electroencephalogr Clin Neurophysiol (1997) 104:257-68. doi: 10.1016/0013-4694(70)90124-0

177. Kutas M, Federmeier KD. Thirty years and counting: finding meaning in the N400 component of the event-related brain potential (ERP). Annu Rev Psychol (2011) 62:621-47. doi: 10.1016/S0168-5597(97)96107-4

178. Stroop JR. Studies of interference in serial verbal reactions. J Exp Psychol (1935) 18:643-62. doi: 10.1146/annurev.psych.093008.131123

179. Falkenstein M, Hoormann J, Hohnsbein J. ERP components in Go/Nogo tasks and their relation to inhibition. Acta Psychol (1999) 101:267-91 doi: $10.1037 / \mathrm{h} 0054651$

180. Enriquez-Geppert S, Konrad C, Pantev C, Huster RJ. Conflict and inhibition differentially affect the N200/P300 complex in a combined go/ nogo and stop-signal task. NeuroImage (2010) 51:877-87. doi: 10.1016/ S0001-6918(99)00008-6

181. Smith JL, Johnstone SJ, Barry RJ. Movement-related potentials in the Go/ NoGo task: the P3 reflects both cognitive and motor inhibition. Clin Neurophysiol (2008) 119:704-14. doi: 10.1016/j.neuroimage.2010.02.043

182. Holroyd CB, Coles MGH. The neural basis of human error processing: reinforcement learning, dopamine, and the error-related negativity. Psychol $\operatorname{Rev}$ (2002) 109:679-709. doi: 10.1016/j.clinph.2007.11.042

183. Galatzer-Levy IR, Bryant RA. 636,120 ways to have posttraumatic stress disorder. Perspect Psychol Sci (2013) 8:651-62. doi: 10.1037/0033-295X.109.4.679

184. Stewart JL, Allen JJ. Resting frontal brain asymmetry is linked to future depressive symptoms in women. Biol Psychology (2018) 136:161-7. doi: 10.1177/1745691613504115

185. Benedek M, Schickel RJ, Jauk E, Fink A, Neubauer AC. Alpha power increases in right parietal cortex reflects focused internal attention. Neuropsychologia (2014) 56:393-400. doi: 10.1016/j.biopsycho.2018.06.004

186. Rabinak CA, Mori S, Lyons M, Milad MR, Phan KL. Acquisition of CS-US contingencies during Pavlovian fear conditioning and extinction in social anxiety disorder and posttraumatic stress disorder. J Affect Disord (2017) 207:76-85. doi: 10.1016/j.neuropsychologia.2014.02.010

187. van Rooij SJ, Kennis M, Vink M, Geuze E. Predicting treatment outcome in PTSD: a longitudinal functional MRI study on trauma-unrelated emotional processing. Neuropsychopharmacology (2016) 41:1156-65. doi: 10.1016/j. jad.2016.09.018

188. Jak AJ, Aupperle R, Rodgers CS, Lang AJ, Schiehser DM, Norman SB, et al. Evaluation of a hybrid treatment for veterans with comorbid traumatic brain injury and posttraumatic stress disorder: study protocol for a randomized controlled trial. Contemp Clin Trials (2015) 45:210-6. doi: 10.1038/ npp. 2015.257

189. Aupperle RL, Melrose AJ, Stein MB, Paulus MP. Executive function and PTSD: disengaging from trauma. Neuropharmacology (2012) 62:686-94. doi: 10.1016/j.cct.2015.10.009

190. Hochberger WC, Joshi YB, Thomas ML, Zhang W, Bismark AW, Treichler $\mathrm{EB}$, et al. Neurophysiologic measures of target engagement predict response to auditory-based cognitive training in treatment refractory schizophrenia. Neuropsychopharmacology (2019) 44:606-12. doi: 10.1016/j. neuropharm.2011.02.008
191. Light GA, Swerdlow NR. Future clinical uses of neurophysiological biomarkers to predict and monitor treatment response for schizophrenia. Ann NY Acad Sci (2015) 1344:105-19. doi: 10.1038/s41386-018-0256-9

192. Houston RJ, Schlienz NJ. Event-related potentials as biomarkers of behavior change mechanisms in substance use disorder treatment. Biol Psychiatry Cogn Neurosci Neuroimaging (2018) 3:30-40. doi: 10.1111/nyas.12730

193. Rabe S, Zöllner T, Beauducel A, Maercker A, Karl A. Changes in brain electrical activity after cognitive behavioral therapy for posttraumatic stress disorder in patients injured in motor vehicle accidents. Psychosom Med (2008) 70:13e9. doi: 10.1016/j.bpsc.2017.09.006

194. Kang SS, Erbes CR, Lamberty GJ, Thuras P, Sponheim SR, Polusny MA, et al. Transcendental meditation for veterans with post-traumatic stress disorder. Psychol Trauma: Theory, Research, Practice, and Policy (2018) 10:675-80. doi: 10.1097/PSY.0b013e31815aa325

195. Lowe CJ, Manocchio F, Safati AB, Hall PA. The effects of theta burst stimulation (TBS) targeting the prefrontal cortex on executive functioning: a systematic review and meta-analysis. Neuropsychologia (2018) 111:344-59. doi: $10.1037 /$ tra0000346

196. Berlim MT, McGirr A, dos Santos NR, Tremblay S, Martins R. Efficacy of theta burst stimulation (TBS) for major depression: an exploratory metaanalysis of randomized and sham-controlled trials. J Psychiatr Res (2017) 90:102-9. doi: 10.1016/j.neuropsychologia.2018.02.004

197. Stagg CJ, Nitsche MA. Physiological basis of transcranial direct current stimulation. Neuroscientist (2011) 17:37-53. doi: 10.1016/j. jpsychires.2017.02.015

198. Saunders N, Downham R, Turman B, Kropotov J, Clark R, Yumash R, et al. Working memory training with tDCS improves behavioral and neurophysiological symptoms in pilot group with post-traumatic stress disorder (PTSD) and with poor working memory. Neurocase (2015) 21:2718. doi: 10.1177/1073858410386614

199. Tillman GD, Kimbrell TA, Calley CS, Kraut MA, Freeman TW, Hart J, Jr.. Repetitive transcranial magnetic stimulation and threat memory: selective reduction of combat threat memory p300 response after right frontallobe stimulation. J Neuropsychiatry Clin Neurosci (2011) 23:40-7. doi: 10.1080/13554794.2014.890727

200. Kozel FA, Motes MA, Didehbani N, DeLaRosa B, Bass C, Schraufnagel $\mathrm{CD}$, et al. Repetitive TMS to augment cognitive processing therapy in combat veterans of recent conflicts with PTSD: a randomized clinical trial. J Affect Disord (2018) 229:506-14. doi: 10.1176/appi.neuropsych. 23.1.40

201. Panisch LS, Hai AH. The effectiveness of using neurofeedback in the treatment of post-traumatic stress disorder: a systematic review. Trauma Violence Abuse (2018). doi: 10.1177/1524838018781103

202. Reiter K, Andersen SB, Carlsson J. Neurofeedback treatment and posttraumatic stress disorder: effectiveness of neurofeedback on posttraumatic stress disorder and the optimal choice of protocol. J Nerv Ment Dis (2016) 204:69-77. doi: 10.1177/1524838018781103

203. Kluetsch RC, Ros T, Theberge J, Frewen PA, Calhoun VD, Schmahl C, et al. Plastic modulation of PTSD resting-state networks and subjective wellbeing by EEG neurofeedback. Acta Psychiatr Scand (2014) 130:123-36. doi: 10.1097/NMD.0000000000000418

204. Nicholson AA, Rabellino D, Densmore M, Frewen PA, Paret C, Kluetsch R, et al. Intrinsic connectivity network dynamics in PTSD during amygdala downregulation using real-time fMRI neurofeedback: a preliminary analysis. Human Brain Map (2018) 39:4258-75. doi: 10.3389/fnhum.2018.00521

205. Banks SJ, Eddy KT, Angstadt M, Nathan PJ, Phan KL. Amygdala-frontal connectivity during emotion regulation. Soc Cogn Affect Neurosci (2007) 2(4):303-12. doi: 10.1093/scan/nsm029

206. Zotev V, Phillips R, Misaki M, Wong CK, Wurfel BE, Krueger F, et al. Real-time fMRI neurofeedback training of the amygdala activity with simultaneous EEG in veterans with combat-related PTSD. NeuroImage Clin (2018) 19:106-21. doi: 10.1111/acps.12229

207. van der Kolk BA, Hodgdon H, Gapen M, Musicaro R, Suvak MK, Hamlin E, et al. A randomized controlled study of neurofeedback for chronic PTSD. PLoS One (2016) 11:e0166752. doi: 10.1016/j.nicl.2018.04.010

208. Popov T, Jordanov T, Rockstroh B, Elbert T, Merzenich MM, Miller GA. Specific cognitive training normalizes auditory sensory gating in 
schizophrenia: a randomized trial. Biol Psychiatry (2011) 69:465-71. doi: 10.1371/journal.pone.0166752

209. Perez VB, Tarasenko M, Miyakoshi M, Pianka ST, Makeig SD, Braff DL, et al. Mismatch negativity is a sensitive and predictive biomarker of perceptual learning during auditory cognitive training in schizophrenia. Neuropsychopharmacology (2017) 42:2206-13. doi: 10.1016/j.biopsych. 2010.09.028

210. Nidich S, Mills PJ, Rainforth M, Heppner P, Schneider RH, Rosenthal NE, et al. Non-trauma-focused meditation versus exposure therapy in veterans with post-traumatic stress disorder: a randomised controlled trial. Lancet Psychiatry (2018) 5:975-86. doi: 10.1038/npp.2017.25

211. Wald I, Fruchter E, Ginat K, Stolin E, Dagan D, Bliese PD, et al. Selective prevention of combat-related post-traumatic stress disorder using attention bias modification training: a randomized controlled trial. Psychol Med (2016) 46:2627-36. doi: 10.1016/S2215-0366(18)30384-5

Conflict of Interest Statement: The authors declare that the research was conducted in the absence of any commercial or financial relationships that could be construed as a potential conflict of interest.

Copyright $\odot 2019$ Butt, Espinal, Aupperle, Nikulina and Stewart. This is an openaccess article distributed under the terms of the Creative Commons Attribution License (CC BY). The use, distribution or reproduction in other forums is permitted, provided the original author(s) and the copyright owner(s) are credited and that the original publication in this journal is cited, in accordance with accepted academic practice. No use, distribution or reproduction is permitted which does not comply with these terms. 